\title{
One-pot diastereoselective synthesis of tetrahydroquinolines from star anise oil in choline chloride/zinc chloride eutectic mixture
}

Angélica Peñaranda Gómez, ${ }^{\dagger}$ Oscar Rodríguez Bejarano, ${ }^{\dagger \dagger}$ Vladimir V. Kouznetsov ${ }^{\dagger *}$ and Cristian Ochoa-Puentes ${ }^{\ddagger}$.

† Laboratorio de Química Orgánica y Biomolecular, Escuela de Química, Universidad Industrial de Santander, A. A. 678, Bucaramanga, Colombia.

† Laboratorio de Electroquímica y Termodinámica Computacional, Departamento de Química, Universidad Nacional de Colombia-Sede Bogotá, Carrera 45 \# 26-85, A.A 5997, Bogotá, Colombia.

\$ Laboratorio de Síntesis Orgánica Sostenible, Departamento de Química, Universidad Nacional de Colombia-Sede Bogotá, Carrera 45 \# 26-85, A.A 5997, Bogotá, Colombia.

kouznet@uis.edu.co (V. Kouznetsov),cochoapu@unal.edu.co (C. ochoa-Puentes)

Number of pages: 57

Number of figures: 66

Number of Schemes: 4

Number of equations: 40 
Table of contents

1. General experimental details and characterization data

2. Copies of MS, ${ }^{1} \mathrm{H}$ NMR and ${ }^{13} \mathrm{C}$ NMR of all compounds

3. Green chemistry metrics analysis

4. References 


\section{General experimental details}

All chemicals were of analytical reagent grade and used as received without further purification except for choline chloride $(\mathrm{ChCl})$, which was dried at $130{ }^{\circ} \mathrm{C}$ in vacuo and stored under nitrogen prior to use. Melting points, reported without correction, were measured using a Fisher-Jhones apparatus. The FT-IR spectra were obtained with a Shimadzu IR prestige 21 spectrophotometer (Columbia, MD, USA). ${ }^{1} \mathrm{H}$ and ${ }^{13} \mathrm{C}-\mathrm{NMR}$ spectra were recorded with a Bruker AVANCE III system operating at $400 \mathrm{MHz}$, using residual and deuterated solvent peaks of $\mathrm{CDCl}_{3}\left(\delta_{\mathrm{H}} 7.26 ; \delta_{\mathrm{C}} 77.0\right)$ and DMSO $\left(\delta_{\mathrm{H}} 2.50 ; \delta_{\mathrm{C}} 39.5\right)$ as reference standards. The peak patterns are indicated as follows: s, singlet; $\mathrm{d}$, doublet; $\mathrm{t}$, triplet; $\mathrm{m}$, multiplet; $\mathrm{q}$, quartet. The coupling constants, $J$, are reported in hertz $(\mathrm{Hz})$. Elemental analyses were performed on a Thermo ScientificFlash $2000 \mathrm{CHNS} / \mathrm{O}$ analyser and their results were found to be in agreement with the calculated values. For the DSC analysis, 6 samples of $\mathrm{ChCl} / \mathrm{ZnCl}_{2}$ of different molar compositions $(2.3: 1,1.5: 1,1: 1,1: 1.5,1: 2$ and $1: 2.3)$ were prepared based on the method described previously in the literature. ${ }^{47} \mathrm{~A}$ portion of each warm mixture liquid was loaded into a hermetically sealed $40 \mu \mathrm{L}$ aluminum pan. The sample was then cooled to $10{ }^{\circ} \mathrm{C}$ and then heated to $100{ }^{\circ} \mathrm{C}$ at 1 ${ }^{\circ} \mathrm{C} \min ^{-1}$ under a nitrogen atmosphere $\left(50 \mathrm{~cm}^{3} \mathrm{~min}^{-1}\right)$ on a differential scanning calorimeter DSC 1 STARe System (Mettler Toledo). Essential oils, ethanolic extracts and all synthesized compounds were analyzed by gas chromatography coupled to mass spectrometry (EVOQ GC-TQ SCION 436-GC) equipped with a Rxi-5Sil MS column $(15 \mathrm{~m} \times 0,25 \mathrm{mmlD} \times 0,25 \mu \mathrm{m})$, the ionization mode was electronic impact at $70 \mathrm{eV}$ 


\section{Raw material preparation and isolation of the volatile oil and extracts.}

Commercially available, good-quality whole dried star anise fruits (Illicium verum

Hook fillius) were purchased from the local market of Paloquemao in Bogotá, Cundinamarca, Colombia. For steam distillation and hydrodistillation experiments $200 \mathrm{~g}$ of whole anise seeds were used, while $2 \mathrm{~g}$ of raw material and ethanol (1:10 w/w) were used for soxhlet and ultrasound assisted extraction. All extractions were conducted for 120 minutes in triplicate.

DES preparation. The DESs were formed by mixing the components in the respective molar ratio (Table 1) in a glass vial. The mixtures were heated up to $80{ }^{\circ} \mathrm{C}$ until a homogeneous, colourless liquid was obtained.

General procedure for the synthesis of tetrahydroquinolines $4 \mathrm{a}-\mathrm{v} .0 .8 \mathrm{~g}$ of $\mathrm{ChCl} /$ zinc chloride DES (1.5:1) was heated to $70{ }^{\circ} \mathrm{C}$ to obtain a clear melt. To this melt a mixture of substituted aniline $(1 \mathrm{mmol})$, aromatic aldehyde $(1 \mathrm{mmol})$ and star anise essential oil ( $87 \%$ of trans-anethole content, $1 \mathrm{mmol}$ of trans-anethole) was added and the reaction was stirred at $110^{\circ} \mathrm{C}$ for $3 \mathrm{~h}$. After completion of the reaction (monitored by TLC), the reaction mixture was quenched by adding water while still hot, cooled to room temperature and the crude solid was filtered, washed with water and purified by column chromatography on silica gel (60-120 mesh) using a mixture of petroleum ether-ethyl acetate as eluent to afford the tetrahydroquinoline derivatives.

\section{Cis-4-(4-methoxyphenyl)-3,6-dimethyl-2-phenyl-1,2,3,4-tetrahydroquinoline}

(4a): white solid (0.29 g, $0.85 \mathrm{mmol}, 85 \%)$; $\mathrm{R}_{f}[$ hexane-EtOAc 15:1] $=0.50$; m.p. $208-210^{\circ} \mathrm{C} ; \mathrm{IR}(\mathrm{KBr}) v_{\max }=3373,2889,2958,1610,1505,1242 \mathrm{~cm}^{-1} ;{ }^{1} \mathrm{H} \mathrm{NMR}$ 
(400 $\left.\mathrm{MHz}_{\mathrm{CDCl}}\right), \delta(\mathrm{ppm}): 7.48-7.41\left(2 \mathrm{H}, \mathrm{m}, 2^{\prime}, 6\right.$ '-HAr), $7.42-7.33(2 \mathrm{H}, \mathrm{m}$, 3',5'-HAr ), 7.32 (1H, t, $J=7.1 \mathrm{~Hz}, 4$ '-HAr), 7.18 - 7.11 (2H, m, 3',5”-HAr), 6.92 $6.84(2 \mathrm{H}, \mathrm{m}, 2$ "',6”-HAr), $6.81(1 \mathrm{H}, \mathrm{dd}, J=7.9,2.2 \mathrm{~Hz}, 7-\mathrm{H}), 6.47(1 \mathrm{H}, \mathrm{d}, J=8.0$ Hz, 8-H), $6.41(1 \mathrm{H}, \mathrm{s}, 5-\mathrm{H}), 4.09(1 \mathrm{H}, \mathrm{d}, J=9.9 \mathrm{~Hz}, 4-\mathrm{H}), 3.97$ (Br s, N-H), 3.83 $\left(3 \mathrm{H}, \mathrm{s}, 4^{\prime}-\mathrm{OCH}_{3}\right), 3.72(1 \mathrm{H}, \mathrm{d}, \mathrm{J}=10.8 \mathrm{~Hz}, 2-\mathrm{H}), 2.18(1 \mathrm{H}, \mathrm{td}, J=10.4,6.5 \mathrm{~Hz}, 3-$ H), $2.10\left(3 \mathrm{H}, \mathrm{s}, 6-\mathrm{CH}_{3}\right), 0.57\left(3 \mathrm{H}, \mathrm{d}, J=6.5 \mathrm{~Hz}, 3-\mathrm{CH}_{3}\right) ;{ }^{13} \mathrm{C} \mathrm{NMR}(101 \mathrm{MHz}$, $\left.\mathrm{CDCl}_{3}\right), \delta(\mathrm{ppm}): 158.1,142.9,142.8,136.9,130.5,130.4(2), 128.5$ (2), 127.9 (2), $127.8,127.5,126.7,125.8,113.8,113.7$ (2), 64.1, 55.2, 51.7, 42.0, 20.5, 16.5; GCMS (EI) $\left(t_{R}=11.30 \mathrm{~min}\right), \mathrm{m} / \mathrm{z} 343.2\left(\mathrm{M}^{+}\right)$; Anal. calcd for $\mathrm{C}_{24} \mathrm{H}_{25} \mathrm{NO}: \mathrm{C}, 83.93 ; \mathrm{H}$, 7.34; N, 4.08, found: C, 83.78; H, 7.32; N, 4.03.

Cis-4-(4-methoxyphenyl)-3-methyl-2-phenyl-1,2,3,4-tetrahydroquinoline (4b): white solid (0.18 g, 0.48 mmol, $55 \%) \mathrm{R}_{f}[$ hexane-EtOAc 15:1] = 0.40; m.p. 135 $137^{\circ} \mathrm{C}$; IR $(\mathrm{KBr}) v_{\max }=3366,2894,2968,1601,1509,1240 \mathrm{~cm}^{-1} ;{ }^{1} \mathrm{H}$ NMR $(400$ $\left.\mathrm{MHz}_{\mathrm{CDCl}}\right), \delta(\mathrm{ppm}): 7.45\left(2 \mathrm{H}, \mathrm{d}, J=6.7 \mathrm{~Hz}, 2^{\prime}, 6\right.$ ' $\left.-\mathrm{HAr}\right), 7.38(2 \mathrm{H}, \mathrm{t}, J=7.1 \mathrm{~Hz}$, 3',5'-HAr), 7.37 - 7.28 (1H, m, 4'-HAr), 7.15 (2H, d, J = 8.6 Hz, 3', 5''-HAr), 7.04 $6.95(1 \mathrm{H}, \mathrm{m}, 7-\mathrm{H}), 6.88$ (2H, d, $J=8.7 \mathrm{~Hz}, 2$ "',6"-HAr), $6.62-6.53(2 \mathrm{H}, \mathrm{m}, 5,6-\mathrm{H})$, $6.53(1 \mathrm{H}, \mathrm{d}, J=7.7 \mathrm{~Hz}, 8-\mathrm{H}), 4.13(1 \mathrm{H}, \mathrm{d}, J=9.9 \mathrm{~Hz}, 4-\mathrm{H}), 4.08(\mathrm{~s}, \mathrm{~N}-\mathrm{H}), 3.82(3 \mathrm{H}$, s, 4"-OCH $\left.{ }_{3}\right), 3.75(1 \mathrm{H}, \mathrm{d}, \mathrm{J}=10.9 \mathrm{~Hz}, 2-\mathrm{H}), 2.20(1 \mathrm{H}, \mathrm{ddd}, J=10.9,9.9,6.5 \mathrm{~Hz}, 3-$ $\mathrm{H}), 0.58\left(3 \mathrm{H}, \mathrm{d}, \mathrm{J}=6.6 \mathrm{~Hz}, 3-\mathrm{CH}_{3}\right) ;{ }^{13} \mathrm{C} \mathrm{NMR}\left(101 \mathrm{MHz}, \mathrm{CDCl}_{3}\right), \delta(\mathrm{ppm}): 158.2$, $145.1,142.9,136.7,130.4(2), 130.2,128.6(2), 128.0(3), 127.0,125.8,117.5,113.8$ (2), 113.7, 63.9, 55.3 51.7, 41.7, 16.5. GC-MS (EI) $\left(t_{R}=8.82 \mathrm{~min}\right), \mathrm{m} / \mathrm{z} 329.1\left(\mathrm{M}^{+}\right)$; Anal. calcd for $\mathrm{C}_{23} \mathrm{H}_{23} \mathrm{NO}$ : C, 83.85; H, 7.04; N, 4.25, found: C, 83.76; H, 7.01; N, 4.23. 
Cis-4-(4-methoxyphenyl)-3-methyl-2-phenyl-1,2,3,4-

tetrahydrobenzo[h]quinoline (4c): yellow solid $(0.18 \mathrm{~g}, 0.48 \mathrm{mmol}, 53 \%) \mathrm{R}_{f}$ $\left[\right.$ hexane-EtOAc 15:1] $=0.36$; m.p. $145-147^{\circ} \mathrm{C}$; IR $(\mathrm{KBr}) v_{\max }=3399,2832,2952$, 1608, 1509, $1242 \mathrm{~cm}^{-1} ;{ }^{1} \mathrm{H}$ NMR (400 MHz, $\left.\mathrm{CDCl}_{3}\right), \delta(\mathrm{ppm}): 7.72(2 \mathrm{H}, \mathrm{td}, J=9.4$, $2.5 \mathrm{~Hz}, 7,10-\mathrm{H}), 7.54(2 \mathrm{H}, \mathrm{d}, J=6.9 \mathrm{~Hz}, 8,9-\mathrm{H}), 7.46-7.34\left(5 \mathrm{H}, \mathrm{m}, 2^{\prime}, 3^{\prime}, 4^{\prime}, 5^{\prime}, 6^{\prime}-\right.$ HAr), 7.15 (2H, d, $J=8.6 \mathrm{~Hz}, 3$ 3"5"-HAr), $7.10(1 \mathrm{H}, \mathrm{d}, J=8.5 \mathrm{~Hz}, 6-\mathrm{H}), 6.88$ (2H, d, $J=8.7 \mathrm{~Hz}, 2$ ",6"-HAr), $6.80(1 \mathrm{H}, \mathrm{d}, J=8.5 \mathrm{~Hz}, 5-\mathrm{H}), 4.72(\mathrm{~s}, \mathrm{~N}-\mathrm{H}), 4.22(1 \mathrm{H}, \mathrm{d}$, $J=10.1 \mathrm{~Hz}, 4-\mathrm{H}), 3.94(1 \mathrm{H}, \mathrm{d}, J=10.6 \mathrm{~Hz}, 2-\mathrm{H}), 3.82\left(3 \mathrm{H}, \mathrm{s}, 4{ }^{\prime \prime}-\mathrm{OCH}_{3}\right), 2.35-$ $2.23(1 \mathrm{H}, \mathrm{m}, 3-\mathrm{H}), 0.66\left(3 \mathrm{H}, \mathrm{d}, J=6.5 \mathrm{~Hz}, 3-\mathrm{CH}_{3}\right) ;{ }^{13} \mathrm{C} \mathrm{NMR}\left(101 \mathrm{MHz}, \mathrm{CDCl}_{3}\right), \delta$ (ppm): 158.2, 142.8, 139.8, 137.3, 132.9, 130.5 (2), 128.8 (2), 128.6, 128.6, 128.2 (2), 128.1, 125.4, 125.0,119.8, 117.3, 113.9 (2), 64.1, 55.3, 52.1, 42.3, 16.6; GC-MS (EI) $\left(t_{R}=17.17 \mathrm{~min}\right), \mathrm{m} / \mathrm{z} 379.1\left(\mathrm{M}^{+}\right)$; Anal. calcd for $\mathrm{C}_{27} \mathrm{H}_{25} \mathrm{NO}$ : C, 85.45; H, 6.64; $\mathrm{N}, 3.69$, found: C, 85.40; H, 6.59; N, 3.65.

\section{Cis-6-methoxy-4-(4-methoxyphenyl)-3-methyl-2-phenyl-1,2,3,4-}

tetrahydroquinoline (4d): light yellow solid $(0.15 \mathrm{~g}, 0.42 \mathrm{mmol}, 46 \%) \mathrm{R}_{f}$ [hexaneEtOAc 15:1] $=0.36$; m.p. $170-172{ }^{\circ} \mathrm{C}$; IR $(\mathrm{KBr}) v_{\max }=3366,2831,2956,1608$, 1501, $1239 \mathrm{~cm}^{-1} ;{ }^{1} \mathrm{H}$ NMR (400 MHz, $\left.\mathrm{CDCl}_{3}\right), \delta(\mathrm{ppm}): 7.46-7.41\left(2 \mathrm{H}, \mathrm{m}, 2^{\prime}, 6^{\prime}-\right.$ HAr), 7.40 - $7.34\left(2 \mathrm{H}, \mathrm{m}, 3^{\prime}, 5^{\prime}\right.$-HAr $), 7.32\left(1 \mathrm{H}, \mathrm{m}, 4^{\prime}-\mathrm{HAr}\right), 7.16$ - $7.11(2 \mathrm{H}, \mathrm{m}$, 3",5"-HAr), $6.89-6.84$ (2H, m, 2",6"-HAr), 6.61 (1H, dd, $J=8.6,2.9$ Hz, 7-H), $6.50(1 \mathrm{H}, \mathrm{d}, J=8.6 \mathrm{~Hz}, 8-\mathrm{H}), 6.20(1 \mathrm{H}, \mathrm{dd}, J=2.8,0.8 \mathrm{~Hz}, 5-\mathrm{H}), 4.06(1 \mathrm{H}, \mathrm{d}, J=$ $9.9 \mathrm{~Hz}, 4-\mathrm{H}), 3.88$ (s, N-H), $3.81\left(3 \mathrm{H}, \mathrm{s}, 4\right.$ "'- $\left.\mathrm{OCH}_{3}\right), 3.73(1 \mathrm{H}, \mathrm{d}, J=10.8 \mathrm{~Hz}, 2-\mathrm{H})$, $3.59\left(3 \mathrm{H}, \mathrm{s}, 6-\mathrm{OCH}_{3}\right), 2.24-2.13(1 \mathrm{H}, \mathrm{m}, 3-\mathrm{H}), 0.57\left(3 \mathrm{H}, \mathrm{d}, J=6.5 \mathrm{~Hz}, 3-\mathrm{CH}_{3}\right) ;{ }^{13} \mathrm{C}$ NMR (101 MHz, $\left.\mathrm{CDCl}_{3}\right), \delta(\mathrm{ppm}): 158.2,152.0,143.0,139.5,136.7,130.4$ (2), 
$128.6(2), 128.0$ (2), 127.9, 127.3, 116.0, 114.7, 113.9 (2), 112.9, 64.3, 55.8, 55.3, 52.1, 42.1, 16.6; GC-MS (EI) $\left(\mathrm{t}_{\mathrm{R}}=11.92 \mathrm{~min}\right), \mathrm{m} / \mathrm{z} 259.2\left(\mathrm{M}^{+}\right)$; Anal. calcd for $\mathrm{C}_{24} \mathrm{H}_{25} \mathrm{NO}_{2}: \mathrm{C}, 80.19 ; \mathrm{H}, 7.01 ; \mathrm{N}, 3.90$, found: C, 79.98; H, 6.98; N, 3.90.

Cis-6-bromo-4-(4-methoxyphenyl)-3-methyl-2-phenyl-1,2,3,4-

tetrahydroquinoline (4e): white solid $(0.25 \mathrm{~g}, 0.61 \mathrm{mmol}, 66 \%) \mathrm{R}_{f}$ [hexane-EtOAc 15:1] $=0.40$; m.p. $216-217{ }^{\circ} \mathrm{C}$; IR $(\mathrm{KBr}) v_{\max }=3366,2828,2954,1591,1489$, $1239 \mathrm{~cm}^{-1} ;{ }^{1} \mathrm{H}$ NMR (400 MHz, $\left.\mathrm{CDCl}_{3}\right), \delta(\mathrm{ppm}): 7.45$ - 7.36 (2H, m, 2', $\left.6^{\prime}-\mathrm{HAr}\right)$, $7.39-7.28$ (3H, m, 3', 5', 4'-HAr), 7.11 (2H, d, $J=8.7 \mathrm{~Hz}, 3$ ', 5'-HAr), 7.05 (1H, dd, $J=8.5,1.5 \mathrm{~Hz}, 7-\mathrm{H}), 6.88(2 \mathrm{H}, \mathrm{d}, J=8.7 \mathrm{~Hz}, 2 ", 6 "-H A r), 6.65$ (1H, dd, $J=2.2,1.1$ Hz, 5-H), 6.39 (1H, d, $J=8.5 \mathrm{~Hz}, 8-\mathrm{H}), 4.09(2 \mathrm{H}, \mathrm{d}, J=9.9 \mathrm{~Hz}, 4-\mathrm{H}, \mathrm{N}-\mathrm{H}), 3.82$ $(3 \mathrm{H}, \mathrm{s}, 4$ "'-OCH 3$), 3.68(1 \mathrm{H}, \mathrm{d}, J=10.9 \mathrm{~Hz}, 2-\mathrm{H}), 2.14(1 \mathrm{H}, \mathrm{td}, J=10.8,10.4,6.5$ $\mathrm{Hz}, 3-\mathrm{H}), 0.55\left(3 \mathrm{H}, \mathrm{d}, J=6.5 \mathrm{~Hz}, 3-\mathrm{CH}_{3}\right) ;{ }^{13} \mathrm{C} \mathrm{NMR}\left(101 \mathrm{MHz}, \mathrm{CDCl}_{3}\right), \delta(\mathrm{ppm})$ : $158.4,144.1,142.5,135.7,132.5,130.4$ (2), 129.7, 128.7 (2), 128.1, 127.9 (3), 115.2, $114.1(2), 109.1,63.9,55.3,51.5,41.3,16.5$; GC-MS (EI) $\left(t_{R}=13.75 \mathrm{~min}\right)$, $\mathrm{m} / \mathrm{z} 407.0\left(\mathrm{M}^{+}\right)$; Anal. calcd for $\mathrm{C}_{23} \mathrm{H}_{22} \mathrm{BrNO}$ : $\mathrm{C}, 67.65 ; \mathrm{H}, 5.43 ; \mathrm{N}, 3.43$, found: $\mathrm{C}$, 66.94; H, 5.39; N, 3.40.

Cis-6-chloro-4-(4-methoxyphenyl)-3-methyl-2-phenyl-1,2,3,4-

tetrahydroquinoline (4f): light yellow solid $(0.26 \mathrm{~g}, 0.71 \mathrm{mmol}, 78 \%) \mathrm{R}_{f}$ [hexaneEtOAc 15:1] $=0.30$; m.p. $215-216^{\circ} \mathrm{C}$; IR $(\mathrm{KBr}) v_{\max }=3366,2894,2955,1591$, 1511, 1491, $1240 \mathrm{~cm}^{-1} ;{ }^{1} \mathrm{H}$ NMR (400 MHz, $\left.\mathrm{CDCl}_{3}\right), \delta(\mathrm{ppm}): 7.44-7.40(2 \mathrm{H}, \mathrm{m}$, 2',6'-HAr), 7.37 (2H, t, $\left.J=7.1 \mathrm{~Hz}, 3^{\prime}, 5^{\prime}-\mathrm{HAr}\right), 7.37$ - 7.28 (1H, m, 4'-HAr), 7.11 $(2 \mathrm{H}, \mathrm{d}, J=8.7 \mathrm{~Hz}, 3$ ", 5"-HAr), $6.92(1 \mathrm{H}, \mathrm{dd}, J=8.6,1.8 \mathrm{~Hz}, 7-\mathrm{H}), 6.88(2 \mathrm{H}, \mathrm{d}, J=$ $8.7 \mathrm{~Hz}, 2$ ", 6"-HAr), $6.53(1 \mathrm{H}, \mathrm{dd}, J=2.3,1.0 \mathrm{~Hz}, 5-\mathrm{H}), 6.44(1 \mathrm{H}, \mathrm{d}, J=8.5 \mathrm{~Hz}, 8-$ 
H), $4.09(2 \mathrm{H}, \mathrm{d}, J=9.9 \mathrm{~Hz}, 4-\mathrm{H}, \mathrm{N}-\mathrm{H}), 3.83\left(3 \mathrm{H}, \mathrm{s}, 4\right.$ "- $\left.-\mathrm{OCH}_{3}\right), 3.68(1 \mathrm{H}, \mathrm{d}, J=11.0$

$\mathrm{Hz}, 2-\mathrm{H}), 2.15(1 \mathrm{H}, \mathrm{td}, J=10.5,6.5 \mathrm{~Hz}, 3-\mathrm{H}), 0.56(3 \mathrm{H}, \mathrm{d}, J=6.5 \mathrm{~Hz}, 3-\mathrm{H}) ;{ }^{13} \mathrm{C}$

NMR (101 MHz, $\left.\mathrm{CDCl}_{3}\right), \delta(\mathrm{ppm}): 158.4,143.7,142.5,135.7,130.4$ (2), 129.7, 128.7 (2), 128.1, 127.9 (2), 127.4, 126.9, 121.9, 114.8, 114.1 (2), 63.9, 55.3, 51.5, 41.4, 16.5; GC-MS (EI) $\left(\mathrm{t}_{\mathrm{R}}=12.45 \mathrm{~min}\right), \mathrm{m} / \mathrm{z} 363.1(\mathrm{M}+)$; Anal. calcd for $\mathrm{C}_{23} \mathrm{H}_{22} \mathrm{ClNO}$ : C, 75.92; H, 6.09; N, 3.85, found: C, 75.47; H, 5.98; N, 3.83.

\section{Cis-4-(4-methoxyphenyl)-3-methyl-6-nitro-2-phenyl-1,2,3,4-tetrahydroquinoline}

(4g): yellow solid (0.34 g, $0.91 \mathrm{mmol}, 98 \%) \mathrm{R}_{f}$ [hexane-EtOAc 15:1] = 0.20; m.p. $175-180^{\circ} \mathrm{C}$; IR $(\mathrm{KBr}) v_{\max }=3339,2836,2956,1603,1504,1290,1242 \mathrm{~cm}^{-1} ;{ }^{1} \mathrm{H}$ NMR (400 MHz, $\left.\mathrm{CDCl}_{3}\right), \delta(\mathrm{ppm}): 7.90(1 \mathrm{H}, \mathrm{dd}, J=8.6,2.3 \mathrm{~Hz}, 7-\mathrm{H}), 7.47(1 \mathrm{H}, \mathrm{dd}$, $J=2.6,1.3 \mathrm{~Hz}, 5-\mathrm{H}), 7.42-7.34$ (5H, m, 2', 3', 4', '5',6'-HAr), 7.15 - 7.06 (2H, m, 3",5"-HAr), 6.90 (2H, d, $J=8.7 \mathrm{~Hz}, 2$ ",6"-HAr), 6.44 (1H, d, $J=8.9 \mathrm{~Hz}, 8-\mathrm{H}), 4.88$ (s, N-H), $4.23(1 \mathrm{H}, \mathrm{d}, J=10.0 \mathrm{~Hz}, 4-\mathrm{H}), 3.83(3 \mathrm{H}, \mathrm{s}, 4$ "'-OCH $), 3.70(1 \mathrm{H}, \mathrm{d}, J=$ $11.2 \mathrm{~Hz}, 2-\mathrm{H}), 2.15(1 \mathrm{H}, \mathrm{ddd}, J=11.2,10.0,6.5 \mathrm{~Hz}, 3-\mathrm{H}), 0.58(3 \mathrm{H}, \mathrm{d}, J=6.5 \mathrm{~Hz}$, 3- $\left.\mathrm{CH}_{3}\right) ;{ }^{13} \mathrm{C}$ NMR (101 MHz, $\left.\mathrm{CDCl}_{3}\right), \delta(\mathrm{ppm}): 158.7,150.3,141.3,138.0,133.9$, $130.2,129.0$ (3), 128.6, 127.8 (2), 126.7, 124.9, 124.4, 114.4 (2), 112.3, 63.7, 55.3, 50.7, 40.2, 16.3; GC-MS (EI) $\left(\mathrm{t}_{\mathrm{R}}=18.50 \mathrm{~min}\right), \mathrm{m} / \mathrm{z} 390.1\left(\mathrm{M}^{+}\right)$; Anal. calcd for $\mathrm{C}_{23} \mathrm{H}_{22} \mathrm{~N}_{2} \mathrm{O}_{3}:$ C, 73.78; H, 5.92; N, 7.48, found: C, 73.70; H, 5.41; N, 7.40.

\section{Cis-4-(4-methoxyphenyl)-3,8-dimethyl-2-phenyl-1,2,3,4-tetrahydroquinoline}

(4h): yellow-orange solid (0.19 g, $0.54 \mathrm{mmol}, 59 \%) \mathrm{R}_{f}$ [hexane-EtOAc 15:1] = 0.43 ; m.p. $123-125^{\circ} \mathrm{C}$; IR (KBr) $v_{\max }=3394,2896,2952,1595,1512,1470,1242$ $\mathrm{cm}^{-1} ;{ }^{1} \mathrm{H}$ NMR (400 MHz, $\mathrm{CDCl}_{3}$ ), $\delta$ (ppm): 7.52 - 7.45 (2H, m, 2', $\left.6^{\prime}-\mathrm{HAr}\right), 7.45-$ 7.26 (3H, m, 3', 4', 5'-HAr), $7.18-7.10$ (2H, m, 3", 5'-HAr), 6.92 (1H, d, $J=4.8 \mathrm{~Hz}$, 
6-H), $6.92-6.84$ (2H, m, 2",6"-HAr), $6.55-6.44(2 \mathrm{H}, \mathrm{m}, 5,7-\mathrm{H}), 4.17(1 \mathrm{H}, \mathrm{d}, J=$ $10.0 \mathrm{~Hz}, 4-\mathrm{H}), 3.94(\mathrm{~s}, \mathrm{~N}-\mathrm{H}), 3.82(3 \mathrm{H}, \mathrm{s}, 4$ "'-OCH 3$), 3.79(1 \mathrm{H}, \mathrm{d}, J=11.0 \mathrm{~Hz}, 2-\mathrm{H})$,

$2.24-2.17(1 \mathrm{H}, \mathrm{m}, 3-\mathrm{H}), 2.12\left(3 \mathrm{H}, \mathrm{s}, 8-\mathrm{CH}_{3}\right), 0.58\left(3 \mathrm{H}, \mathrm{d}, J=6.5 \mathrm{~Hz}, 3-\mathrm{CH}_{3}\right) ;{ }^{13} \mathrm{C}$ NMR (101 MHz, $\left.\mathrm{CDCl}_{3}\right), \delta(\mathrm{ppm}):$ 158.2, 143.2, 137.0, 130.4 (2), 128.7 (2), 128.1

(2), 128.1 (2), 128.0, 125.3, 120.5, 116.8, 113.8 (2), 113.6, 64.1, 55.3, 51.9, 41.5, 17.5, 16.6; GC-MS (EI) $\left(\mathrm{t}_{\mathrm{R}}=9.37 \mathrm{~min}\right), \mathrm{m} / \mathrm{z} 343.2\left(\mathrm{M}^{+}\right)$; Anal. calcd for $\mathrm{C}_{24} \mathrm{H}_{25} \mathrm{NO}$ : C, 83.93; H, 7.34; N, 4.08, found: C, 83.80; H, 7.29; N, 4.01.

Cis-8-methoxy-4-(4-methoxyphenyl)-3-methyl-2-phenyl-1,2,3,4-

tetrahydroquinoline (4i): white solid $(0.13 \mathrm{~g}, 0.36 \mathrm{mmol}, 39 \%) \mathrm{R}_{f}$ [hexane-EtOAc 15:1] $=0.40$; m.p. $209-210^{\circ} \mathrm{C}$; IR $(\mathrm{KBr}) v_{\max }=3404,2832,2954,1610,1509$, $1249 \mathrm{~cm}^{-1} ;{ }^{1} \mathrm{H}$ NMR (400 MHz, $\left.\mathrm{CDCl}_{3}\right), \delta$ (ppm): 7.51 - 7.44 (2H, m, 2',6'-HAr), 7.39 (2H, t, $\left.J=7.2 \mathrm{~Hz}, 3^{\prime}, 5^{\prime}-\mathrm{HAr}\right), 7.37-7.30$ (1H, m, 4'-HAr), 7.15 (2H, d, $J=8.6$ Hz, 3",5"-HAr), 6.87 (2H, d, $J=8.7 \mathrm{~Hz}, 2$ ",6"-HAr), 6.64 (1H, d, $J=8.6 \mathrm{~Hz}, 7-\mathrm{H})$, $6.53(1 \mathrm{H}, \mathrm{t}, J=7.9 \mathrm{~Hz}, 6-\mathrm{H}), 6.25(1 \mathrm{H}, \mathrm{dt}, J=7.8,1.1 \mathrm{~Hz}, 5-\mathrm{H}), 4.59(\mathrm{~s}, \mathrm{~N}-\mathrm{H}), 4.09$ $(1 \mathrm{H}, \mathrm{d}, J=9.9 \mathrm{~Hz}, 4-\mathrm{H}), 3.82\left(6 \mathrm{H}, \mathrm{d}, J=1.8 \mathrm{~Hz}, 4 ", 8-\mathrm{OCH}_{3}\right), 3.78(1 \mathrm{H}, \mathrm{d}, J=10.9$ $\mathrm{Hz}, 2-\mathrm{H}), 2.22(1 \mathrm{H}, \mathrm{ddd}, J=11.4,10.3,6.9 \mathrm{~Hz}, 3-\mathrm{H}), 0.59(3 \mathrm{H}, \mathrm{d}, J=6.5 \mathrm{~Hz}, 3-$ $\left.\mathrm{CH}_{3}\right) ;{ }^{13} \mathrm{C}$ NMR (101 MHz, $\left.\mathrm{CDCl}_{3}\right), \delta(\mathrm{ppm}): 158.2,145.8,142.9,137.0,135.2$, 130.4 (2), 128.6 (2), 128.1 (2), 127.9, 125.9, 122.2, 116.3, 113.8 (2), 107.3, 63.6, 55.5, 55.3, 51.7, 41.9, 16.6; GC-MS (EI) ( $\left.\mathrm{t}_{\mathrm{R}}=11.34 \mathrm{~min}\right), \mathrm{m} / \mathrm{z} 359.2\left(\mathrm{M}^{+}\right)$; Anal. calcd for $\mathrm{C}_{24} \mathrm{H}_{25} \mathrm{NO}_{2}$ : C, 80.19; H, 7.01; N, 3.90, found: C, 79.96; H, 6.97; N, 3.87.

\section{Cis-8-bromo-4-(4-methoxyphenyl)-3-methyl-2-phenyl-1,2,3,4-}

tetrahydroquinoline (4j): white solid $(0.19 \mathrm{~g}, 0.45 \mathrm{mmol}, 49 \%) \mathrm{R}_{f}$ [hexane-EtOAc $15: 1]=0.46$; m.p. $165-166^{\circ} \mathrm{C}$; IR $(\mathrm{KBr}) v_{\max }=3396,2831,2955,1608,1511$, 
1479, $1245 \mathrm{~cm}^{-1} ;{ }^{1} \mathrm{H}$ NMR (400 MHz, $\left.\mathrm{CDCl}_{3}\right), \delta$ (ppm): 7.50 - 7.43 (2H, m, 2',6'HAr), 7.45 - 7.36 (2H, m, 3', 5'-HAr), 7.37 - 7.32 (1H, m, 4'-HAr), 7.30 - 7.22 (1H, m, 7-H), 7.12 (2H, d, $J=8.7 \mathrm{~Hz}, 3$ 3",5"-HAr), 6.88 (2H, d, $J=8.7 \mathrm{~Hz}, 2$ ",6"-HAr), $6.51(1 \mathrm{H}, \mathrm{d}, J=7.6 \mathrm{~Hz}, 6-\mathrm{H}), 6.40(1 \mathrm{H}, \mathrm{t}, J=7.8 \mathrm{~Hz}, 5-\mathrm{H}), 4.70(\mathrm{~s}, \mathrm{~N}-\mathrm{H}), 4.17(1 \mathrm{H}$, d, $J=10.0 \mathrm{~Hz}, 4-\mathrm{H}), 3.82(3 \mathrm{H}, \mathrm{s}, 4$ "'-OCH 3$), 3.75(1 \mathrm{H}, \mathrm{d}, \mathrm{J}=11.0 \mathrm{~Hz}, 2-\mathrm{H}), 2.16$ $(1 \mathrm{H}, \mathrm{ddd}, J=11.0,10.0,6.5 \mathrm{~Hz}, 3-\mathrm{H}), 0.57\left(3 \mathrm{H}, \mathrm{d}, J=6.5 \mathrm{~Hz}, 3-\mathrm{CH}_{3}\right) ;{ }^{13} \mathrm{C}$ NMR (101 MHz, $\left.\mathrm{CDCl}_{3}\right), \delta(\mathrm{ppm}): 158.4,142.4,142.2,136.1,130.4$ (2), 130.3, 129.3, 128.8 (2), 128.2, 128.0 (2), 127.4, 117.5, 114.0 (2), 108.3, 63.9, 55.3, 51.8, 41.4, 16.5; GC-MS (EI) ( $\left.\mathrm{t}_{\mathrm{R}}=12.26 \mathrm{~min}\right), \mathrm{m} / \mathrm{z} 407.1\left(\mathrm{M}^{+}\right)$; Anal. calcd for $\mathrm{C}_{23} \mathrm{H}_{22} \mathrm{BrNO}$ : $\mathrm{C}$, 67.65; H, 5.43; N, 3.43, found: C, 67.59; H, 5.34; N, 3.39.

\section{Cis-8-chloro-4-(4-methoxyphenyl)-3-methyl-2-phenyl-1,2,3,4-}

tetrahydroquinoline (4k): white solid $(0.22 \mathrm{~g}, 0.60 \mathrm{mmol}, 66 \%) \mathrm{R}_{f}$ [hexane-EtOAc $15: 1]=0.50$; m.p. $139-140^{\circ} \mathrm{C} ; \mathrm{IR}(\mathrm{KBr}) v_{\max }=3396,2831,2951,1597,1511$, 1481, $1243 \mathrm{~cm}^{-1} ;{ }^{1} \mathrm{H}$ NMR (400 MHz, $\left.\mathrm{CDCl}_{3}\right), \delta(\mathrm{ppm}): 7.47(2 \mathrm{H}, \mathrm{dd}, J=8.2,1.5$ Hz, 2',6'-HAr), $7.45-7.33$ (3H, m, 3',4',5'-HAr), 7.12 (3H, dd, $J=8.7,2.1 \mathrm{~Hz}$, 3",5"-HAr, 7-H), 6.88 (2H, d, $J=8.6 \mathrm{~Hz}, 2$ ",6"-HAr), $6.51-6.44$ (2H, m, 5,6-H), $4.69(\mathrm{~s}, \mathrm{~N}-\mathrm{H}), 4.15(1 \mathrm{H}, \mathrm{d}, J=10.0 \mathrm{~Hz}, 4-\mathrm{H}), 3.80\left(3 \mathrm{H}, \mathrm{s}, 4\right.$ "'- $\left.-\mathrm{OCH}_{3}\right), 3.73(1 \mathrm{H}, \mathrm{d}, J=$ $11.1 \mathrm{~Hz}, 2-\mathrm{H}), 2.21-2.11(1 \mathrm{H}, \mathrm{m}, 3-\mathrm{H}), 0.58\left(3 \mathrm{H}, \mathrm{d}, J=6.5 \mathrm{~Hz}, 3-\mathrm{CH}_{3}\right) ;{ }^{13} \mathrm{C}$ NMR (101 MHz, $\left.\mathrm{CDCl}_{3}\right), \delta(\mathrm{ppm}): 158.4,142.4,141.2,136.1,130.4$ (2), 129.0, 128.7 (2), $128.5,128.2,128.0(2), 127.3,127.0,116.9,114.0(2), 63.7,55.3,51.7,41.3,16.5$; GC-MS (EI) $\left(\mathrm{t}_{\mathrm{R}}=10.75 \mathrm{~min}\right), \mathrm{m} / \mathrm{z} 363.1\left(\mathrm{M}^{+}\right)$; Anal. calcd for $\mathrm{C}_{23} \mathrm{H}_{22} \mathrm{ClNO}: \mathrm{C}$, 75.92; H, 6.09; N, 3.85, found: C, 75.77; H, 5.95; N, 3.79 . 
Cis-4-(4-methoxyphenyl)-3-methyl-8-nitro-2-phenyl-1,2,3,4-tetrahydroquinoline

(4l): yellow solid (0.26 g, $0.70 \mathrm{mmol}, 77 \%) \mathrm{R}_{f}[$ hexane-EtOAc 15:1] = 0.27; m.p. $155-156^{\circ} \mathrm{C}$; IR $(\mathrm{KBr}) v_{\max }=3330,2832,2952,1608,1492,1333,1246 \mathrm{~cm}^{-1} ;{ }^{1} \mathrm{H}$

NMR (400 MHz, $\left.\mathrm{CDCl}_{3}\right), \delta(\mathrm{ppm}): 8.50(\mathrm{~s}, \mathrm{~N}-\mathrm{H}), 8.01(1 \mathrm{H}, \mathrm{ddd}, J=8.6,1.6,1.0$ Hz, 7-H), 7.46 - 7.33 (5H, m, 2', 3', 4', 5',6'-H), 7.13 - 7.07 (2H, m, 3', ,'”-HAr), 6.95 -6.87 (2H, m, 2",6"-HAr), $6.75(1 \mathrm{H}, \mathrm{dd}, J=7.3,0.7 \mathrm{~Hz}, 5-\mathrm{H}), 6.44(1 \mathrm{H}, \mathrm{dd}, J=8.7$, $7.3 \mathrm{~Hz}, 6-\mathrm{H}), 4.32(1 \mathrm{H}, \mathrm{d}, J=10.1 \mathrm{~Hz}, 4-\mathrm{H}), 3.83(3 \mathrm{H}, \mathrm{s}, 4$ ”'-OCH 3$), 3.73(1 \mathrm{H}, \mathrm{d}, J=$ $11.5 \mathrm{~Hz}, 2-\mathrm{H}), 2.15(1 \mathrm{H}, \mathrm{ddd}, J=11.5,10.2,6.4 \mathrm{~Hz}, 3-\mathrm{H}), 0.60(3 \mathrm{H}, \mathrm{d}, J=6.4 \mathrm{~Hz}$, 3- $\left.\mathrm{CH}_{3}\right) ;{ }^{13} \mathrm{C}$ NMR (101 MHz, $\left.\mathrm{CDCl}_{3}\right), \delta(\mathrm{ppm}): 158.7,143.2,141.3,136.1,134.4$, $130.9,130.3$ (2), 129.8, 129.0 (2), 128.6, 127.7 (2), 124.9, 114.8, 114.3 (2), 63.5, 55.3, 51.3, 39.3, 16.5; GC-MS (EI) $\left(t_{R}=14.93 \mathrm{~min}\right), \mathrm{m} / \mathrm{z} 374.1\left(\mathrm{M}^{+}\right)$; Anal. calcd for $\mathrm{C}_{23} \mathrm{H}_{22} \mathrm{~N}_{2} \mathrm{O}_{3}$ : C, 73.78; H, 5.92; N, 7.48, found: C, 73.25; H, 5.84; N, 7.41.

\section{Cis-7-chloro-4-(4-methoxyphenyl)-3-methyl-2-phenyl-1,2,3,4-tetrahydroquinoline}

(4m): white solid (0.23 g, $0.62 \mathrm{mmol}, 68 \%) \mathrm{R}_{f}[$ hexane-EtOAc 15:1] $=0.33$; m.p.= $157-158^{\circ} \mathrm{C} ; \mathrm{IR}(\mathrm{KBr}) v_{\max }=3396,2835,2955,1600,1238 \mathrm{~cm}^{-1} ;{ }^{1} \mathrm{H}$ NMR (400 $\left.\mathrm{MHz} \mathrm{CDCl}_{3}\right), \delta(\mathrm{ppm}): 7.45-7.29\left(5 \mathrm{H}, \mathrm{m}, 2^{\prime}, 3^{\prime}, 4^{\prime}, 5^{\prime}, 6^{\prime}-\mathrm{HAr}\right), 7.15-7.08(2 \mathrm{H}, \mathrm{m}$, 3",5"-HAr), 6.91 - 6.84 (2H, m, 2",6"-HAr), $6.52-6.43$ (3H, m, 5,6,8-H), 4.11 (2H, d, $J=9.9 \mathrm{~Hz}, 4-\mathrm{H}, \mathrm{N}-\mathrm{H}), 3.82\left(3 \mathrm{H}, \mathrm{s}, 4\right.$ "'- $\left.\mathrm{OCH}_{3}\right), 3.67(1 \mathrm{H}, \mathrm{d}, J=10.0 \mathrm{~Hz}, 2-\mathrm{H})$, $2.21-2.07(1 \mathrm{H}, \mathrm{m}, 3-\mathrm{H}), 0.56\left(3 \mathrm{H}, \mathrm{d}, J=6.5 \mathrm{~Hz}, 3-\mathrm{CH}_{3}\right) ;{ }^{13} \mathrm{C}$ NMR $(101 \mathrm{MHz}$ $\left.\mathrm{CDCl}_{3}\right), \delta(\mathrm{ppm}): 158.4,146.0,142.4,136.0,132.4,131.3,130.4$ (2), 128.7 (2), 128.2, 127.9 (2), 124.2, 117.2, 114.0 (2), 113.0, 63.8, 55.3, 51.1, 41.4, 16.4; GC-MS (EI) $\left(\mathrm{t}_{\mathrm{R}}=12.82 \mathrm{~min}\right), \mathrm{m} / \mathrm{z} 363.1\left(\mathrm{M}^{+}\right)$; Anal. calcd for $\mathrm{C}_{23} \mathrm{H}_{22} \mathrm{ClNO}$ : C, 75.92; $\mathrm{H}$, $6.09 ; \mathrm{N}, 3.85$, found: C, 75.87; H, 6.98; N, 3.82. 
Cis-4-(4-methoxyphenyl)-3-methyl-7-nitro-2-phenyl-1,2,3,4-tetrahydroquinoline

(4n): yellow solid (0.10 g, 0.27 mmol, $28 \%) \mathrm{R}_{f}[$ hexane-EtOAc 15:1] = 0.23; m.p. 172- $174^{\circ} \mathrm{C}$; IR $\left.(\mathrm{KBr}) v_{\max }=3373\right), 2841,2959,1607,1509,1335,1245 \mathrm{~cm}^{-1} ;{ }^{1} \mathrm{H}$ NMR (400 MHz, $\left.\mathrm{CDCl}_{3}\right), \delta(\mathrm{ppm}): 7.42$ - 7.34 (5H, m, 2', '3',4',5',6'-HAr), 7.33 $7.30(2 \mathrm{H}, \mathrm{m}, 5,6-\mathrm{H}), 7.14-7.07$ (2H, m, 3",5”-HAr), $6.93-6.86$ (2H, m, 2",6"HAr), $6.66(1 \mathrm{H}, \mathrm{d}, J=8.2 \mathrm{~Hz}, 8-\mathrm{H}), 4.39(\mathrm{~s}, \mathrm{~N}-\mathrm{H}), 4.17(1 \mathrm{H}, \mathrm{d}, J=10.0 \mathrm{~Hz}, 4-\mathrm{H})$, $3.82(3 \mathrm{H}, \mathrm{s}, 4$ "'-OCH 3$), 3.74(1 \mathrm{H}, \mathrm{d}, J=11.1 \mathrm{~Hz}, 2-\mathrm{H}), 2.17(1 \mathrm{H}, \mathrm{ddd}, J=11.0,10.0$, $6.5 \mathrm{~Hz}, 3-\mathrm{H}), 0.59\left(3 \mathrm{H}, \mathrm{d}, J=6.5 \mathrm{~Hz}, 3-\mathrm{CH}_{3}\right) ;{ }^{13} \mathrm{C} \mathrm{NMR}\left(101 \mathrm{MHz}, \mathrm{CDCl}_{3}\right), \delta$ (ppm): 158.6, 147.2, 145.5, 141.8, 135.0, 132.6, 130.7, 130.3, 128.9 (2), 128.4, $127.8(2), 114.2(2), 111.8,107.7,63.7,55.3,51.5,40.8,16.4 ;$ GC-MS (EI) $\left(t_{R}=\right.$ 17.77 $\mathrm{min}), \mathrm{m} / \mathrm{z} 374.1\left(\mathrm{M}^{+}\right)$; Anal. calcd for $\mathrm{C}_{23} \mathrm{H}_{22} \mathrm{~N}_{2} \mathrm{O}_{3}: \mathrm{C}, 73.78 ; \mathrm{H}, 5.92 ; \mathrm{N}, 7.48$, found: C, 73.65; H, 5.87; N, 7.46.

\section{Cis-4-(4-methoxyphenyl)-3-methyl-5-nitro-2-phenyl-1,2,3,4-tetrahydroquinoline}

(4o): yellow solid (0.18 g, $0.50 \mathrm{mmol}, 55 \%) \mathrm{R}_{f}[$ hexane-EtOAc 15:1] = 0.13; m.p. $140-143^{\circ} \mathrm{C}$; IR $(\mathrm{KBr}) v_{\max }=3386,2823,2915,1605,1524,1363,1240 \mathrm{~cm}^{-1} ;{ }^{1} \mathrm{H}$ NMR (400 MHz, $\left.\mathrm{CDCl}_{3}\right), \delta(\mathrm{ppm}): 7.41$ - 7.31 (5H, m, 2', 3', 4', 5', 6'-HAr), 7.08 (1H, t, $J=7.9 \mathrm{~Hz}, 7-\mathrm{H}), 7.01-6.95$ (2H, m, 3",5”-HAr), 6.93 (1H, dd, $J=7.8,1.3 \mathrm{~Hz}, 6-$ H), 6.79 - 6.72 (3H, m, 8-H, 2",6”-HAr), 4.36 (s, N-H), $4.21(1 \mathrm{H}, \mathrm{d}, J=10.2 \mathrm{~Hz}, 4-$ H), $4.01(1 \mathrm{H}, \mathrm{d}, J=10.2 \mathrm{~Hz}, 2-\mathrm{H}), 3.75(3 \mathrm{H}, \mathrm{s}, 4$ "'-OCH $), 2.08-1.97(1 \mathrm{H}, \mathrm{m}, 3-\mathrm{H})$, $0.67\left(3 \mathrm{H}, \mathrm{d}, J=6.5 \mathrm{~Hz}, 3-\mathrm{CH}_{3}\right) ;{ }^{13} \mathrm{C} \mathrm{NMR}\left(101 \mathrm{MHz}, \mathrm{CDCl}_{3}\right), \delta(\mathrm{ppm}): 158.1$, $152.4,148.0,141.3,135.2,130.5$ (2), 128.8 (2), 128.3, 127.9 (2), 127.2, 119.5, 118.2, 114.2, $113.5(2), 62.7,55.2,48.1,45.1,16.2$; GC-MS (EI) $\left(t_{R}=13.68 \mathrm{~min}\right)$, 
m/z 374.1 $\left(\mathrm{M}^{+}\right)$; Anal. calcd for $\mathrm{C}_{23} \mathrm{H}_{22} \mathrm{~N}_{2} \mathrm{O}_{3}: \mathrm{C}, 73.78 ; \mathrm{H}, 5.92 ; \mathrm{N}, 7.48$, found: $\mathrm{C}$, 73.66; H, 5.87; N, 7.45.

Cis-2,4-bis(4-methoxyphenyl)-3,6-dimethyl-1,2,3,4-tetrahydroquinoline

(4p):

yellow solid (0.15 g, 0.48 mmol, $43 \%) \mathrm{R}_{f}[$ hexane-EtOAc 15:1] = 0.20; m.p. $207-$ $210^{\circ} \mathrm{C} ; \mathrm{IR}(\mathrm{KBr}) v_{\max }=3383,2832,2956,1608,1504,1458,1242 \mathrm{~cm}^{-1} ;{ }^{1} \mathrm{H}$ NMR (400 MHz, $\left.\mathrm{CDCl}_{3}\right), \delta(\mathrm{ppm}): 7.39-7.32(2 \mathrm{H}, \mathrm{m}, 3$ 3",5"-HAr), $7.18-7.11(2 \mathrm{H}, \mathrm{m}$, 2",6"'-HAr), $6.94-6.85$ (4H, m, 2',3',5',6'-HAr), $6.81(1 \mathrm{H}, \mathrm{dd}, J=8.1,2.0 \mathrm{~Hz}, 7-$ H), $6.46(1 \mathrm{H}, \mathrm{d}, J=8.0 \mathrm{~Hz}, 8-\mathrm{H}), 6.40(1 \mathrm{H}, \mathrm{s}, 5-\mathrm{H}), 4.04(1 \mathrm{H}, \mathrm{d}, J=9.9 \mathrm{~Hz}, 4-\mathrm{H})$, $3.92(\mathrm{~s}, \mathrm{~N}-\mathrm{H}), 3.83\left(6 \mathrm{H}, \mathrm{d}, J=1.5 \mathrm{~Hz}, 4^{\prime}, 4\right.$ '- $\left.-\mathrm{OCH}_{3}\right), 3.71(1 \mathrm{H}, \mathrm{d}, J=10.9 \mathrm{~Hz}, 2-\mathrm{H})$, $2.20-2.10(1 \mathrm{H}, \mathrm{m}, 3-\mathrm{H}), 2.10\left(3 \mathrm{H}, \mathrm{s}, 6-\mathrm{CH}_{3}\right), 0.56\left(3 \mathrm{H}, \mathrm{d}, J=6.5 \mathrm{~Hz}, 3-\mathrm{CH}_{3}\right) ;{ }^{13} \mathrm{C}$ NMR (101 MHz, $\left.\mathrm{CDCl}_{3}\right), \delta(\mathrm{ppm}): 158.3,158.2,143.0,137.1,135.2,130.5,130.4$ (2), 129.0 (2), 127.6, 126.7, 125.9, 113.9 (2), 113.8 (2), 63.5, 55.4, 55.3, 51.9, 42.2, 20.6, 16.6; GC-MS (EI) ( $\left.\mathrm{t}_{\mathrm{R}}=13.63 \mathrm{~min}\right), \mathrm{m} / \mathrm{z}$ 373.1( $\left.\mathrm{M}^{+}\right)$; Anal. calcd for $\mathrm{C}_{25} \mathrm{H}_{27} \mathrm{NO}_{2}$ : C, 80.40; H, 7.29; N, 3.75, found: C, 80.35; H, 7.21; N, 7.14.

Cis-4-(4-methoxyphenyl)-3,6-dimethyl-2-(2-nitrophenyl)-1,2,3,4-

tetrahydroquinoline (4q): orange solid $(0.12 \mathrm{~g}, 0.30 \mathrm{mmol}, 33 \%) \mathrm{R}_{f}$ [hexane-EtOAc $15: 1]=0.17$; m.p. $130-132^{\circ} \mathrm{C} ; \mathrm{IR}(\mathrm{KBr}) v_{\max }=3362,2835,2911,1610,1505$, 1349, $1243 \mathrm{~cm}^{-1} ;{ }^{1} \mathrm{H}$ NMR (400 MHz, $\left.\mathrm{CDCl}_{3}\right), \delta(\mathrm{ppm}): 7.85(1 \mathrm{H}, \mathrm{dd}, J=8.0,1.4 \mathrm{~Hz}$, 3'-H), $7.72\left(1 \mathrm{H}, \mathrm{dd}, J=8.2,1.3 \mathrm{~Hz}, 6^{\prime}-\mathrm{H}\right), 7.61\left(1 \mathrm{H}, \mathrm{td}, J=7.6,1.3 \mathrm{~Hz}, 4^{\prime}-\mathrm{H}\right), 7.47$ - $7.38(1 \mathrm{H}, \mathrm{m}, 5$ '-H), $7.16-7.07$ (2H, m, 3",5"-HAr), $6.92-6.79$ (2H, m, 2",6"HAr), $6.51(1 \mathrm{H}, \mathrm{d}, J=8.0 \mathrm{~Hz}, 8-\mathrm{H}), 6.41(1 \mathrm{H}, \mathrm{s}, 5-\mathrm{H}), 4.65(1 \mathrm{H}, \mathrm{d}, J=9.9 \mathrm{~Hz}, 4-\mathrm{H})$, $3.82(3 \mathrm{H}, \mathrm{s}, 4$ "'-OCH 3$), 3.78-3.70(2 \mathrm{H}, \mathrm{m}, 2-\mathrm{H}, \mathrm{N}-\mathrm{H}), 2.32-2.21(1 \mathrm{H}, \mathrm{m}, 3-\mathrm{H})$, $2.10\left(3 \mathrm{H}, \mathrm{s}, 6-\mathrm{CH}_{3}\right), 0.58\left(3 \mathrm{H}, \mathrm{d}, J=6.6 \mathrm{~Hz}, 3-\mathrm{CH}_{3}\right) ;{ }^{13} \mathrm{C} \mathrm{NMR}\left(101 \mathrm{MHz}, \mathrm{CDCl}_{3}\right)$, 
$\delta(\mathrm{ppm}): 158.4,150.6,148.0,142.3,136.3,131.0,130.4(2), 129.0(2), 127.9,127.6$ 126.0, $123.8(2), 114.2,114.0(2), 63.6,55.3,51.5,42.3,20.6,16.4$; GC-MS (EI) $\left(t_{R}\right.$ $=14.06 \mathrm{~min}), \mathrm{m} / \mathrm{z} 388.1\left(\mathrm{M}^{+}\right)$; Anal. calcd for $\mathrm{C}_{24} \mathrm{H}_{24} \mathrm{~N}_{2} \mathrm{O}_{3}: \mathrm{C}, 74.21 ; \mathrm{H}, 6.23 ; \mathrm{N}$, 7.21, found: C, 73.95; H, 6.17; N, 7.14.

Cis-4-(4-methoxyphenyl)-3,6-dimethyl-2-(3-nitrophenyl)-1,2,3,4-

tetrahydroquinoline (4r): orange solid (0.12 g, $0.32 \mathrm{mmol}, 35 \%) \mathrm{R}_{f}$ [hexane-EtOAc $15: 1]=0.26$; m.p. $189-191^{\circ} \mathrm{C}$; IR $(\mathrm{KBr}) v_{\max }=3384,2835,2971,1610,1504$, 1349, $1245 \mathrm{~cm}^{-1}$; ${ }^{1} \mathrm{H}$ NMR (400 MHz, $\left.\mathrm{CDCl}_{3}\right), \delta(\mathrm{ppm}): 8.34\left(1 \mathrm{H}, \mathrm{t}, J=2.0 \mathrm{~Hz}, 2^{\prime}-\right.$ H), $8.18\left(1 \mathrm{H}, \mathrm{ddd}, J=8.2,2.4,1.1 \mathrm{~Hz}, 4^{\prime}-\mathrm{H}\right), 7.80\left(1 \mathrm{H}, \mathrm{dt}, J=7.7,1.4 \mathrm{~Hz}, 5^{\prime}-\mathrm{H}\right)$, $7.55\left(1 \mathrm{H}, \mathrm{t}, J=7.9 \mathrm{~Hz}, 6^{\prime}-\mathrm{H}\right), 7.17-7.11(2 \mathrm{H}, \mathrm{m}, 3$ 3",5"-HAr), $6.92-6.85(2 \mathrm{H}, \mathrm{m}$, 2",6"-HAr), 6.84 (1H, dd, $J=8.0,2.0 \mathrm{~Hz}, 7-\mathrm{H}), 6.51(1 \mathrm{H}, \mathrm{d}, J=8.1 \mathrm{~Hz}, 8-\mathrm{H}), 6.43$ $(1 \mathrm{H}, \mathrm{s}, 5-\mathrm{H}), 4.22(1 \mathrm{H}, \mathrm{d}, J=9.9 \mathrm{~Hz}, 4-\mathrm{H}), 4.01(1 \mathrm{H}, \mathrm{s}, \mathrm{N}-\mathrm{H}), 3.83\left(3 \mathrm{H}, \mathrm{s}, 4{ }^{\prime}-\right.$ $\left.\mathrm{OCH}_{3}\right), 3.74(1 \mathrm{H}, \mathrm{d}, J=10.8 \mathrm{~Hz}, 2-\mathrm{H}), 2.27-2.14(1 \mathrm{H}, \mathrm{m}, 3-\mathrm{H}), 2.11(3 \mathrm{H}, \mathrm{s}, 6-$ $\left.\mathrm{CH}_{3}\right), 0.58\left(3 \mathrm{H}, \mathrm{d}, J=6.6 \mathrm{~Hz}, 3-\mathrm{CH}_{3}\right) ;{ }^{13} \mathrm{C} \mathrm{NMR}\left(101 \mathrm{MHz}, \mathrm{CDCl}_{3}\right), \delta(\mathrm{ppm})$ : $158.4,151.0,148.0,142.3,137.0,131.0,130.4$ (2), 128.9 (2), 128.0, 128.6, 126.0, $124.0(2), 114.2,114.0(2), 64.6,55.3,51.5,42.3,20.6,16.5 ; G C-M S(E I)\left(t_{R}=\right.$ $15.86 \mathrm{~min}), \mathrm{m} / \mathrm{z}$ 388.1( $\left(\mathrm{M}^{+}\right)$; Anal. calcd for $\mathrm{C}_{24} \mathrm{H}_{24} \mathrm{~N}_{2} \mathrm{O}_{3}: \mathrm{C}, 74.21 ; \mathrm{H}, 6.23 ; \mathrm{N}, 7.21$, found: $\mathrm{C}, 74.11 ; \mathrm{H}, 6.12 ; \mathrm{N}, 7.10$.

Cis-4-(4-methoxyphenyl)-3,6-dimethyl-2-(4-nitrophenyl)-1,2,3,4-

tetrahydroquinoline (4s): orange solid $(0.14 \mathrm{~g}, 0.36 \mathrm{mmol}, 38 \%) \mathrm{R}_{f}[$ hexane-EtOAc $15: 1]=0.17$; m.p. $180-183^{\circ} \mathrm{C} ; \mathrm{IR}(\mathrm{KBr}) v_{\max }=3360,2832,2916,1605,1504$, 1341, $1246 \mathrm{~cm}^{-1} ;{ }^{1} \mathrm{H}$ NMR $\left(400 \mathrm{MHz}, \mathrm{CDCl}_{3}\right), \delta(\mathrm{ppm}): 8.22(2 \mathrm{H}, \mathrm{d}, J=8.7 \mathrm{~Hz}$, 3',5'-H), 7.62 (2H, d, J = 8.7 Hz, 2',6'-H), 7.16 - 7.08 (2H, m, 3', 5'-HAr), $6.91-$ 
$6.85(2 \mathrm{H}, \mathrm{m}, 2$ "), $"-\mathrm{HAr}), 6.51(1 \mathrm{H}, \mathrm{d}, J=8.0 \mathrm{~Hz}, 8-\mathrm{H}), 6.42(1 \mathrm{H}, \mathrm{s}, 5-\mathrm{H}), 4.22(1 \mathrm{H}$, d, $J=9.8 \mathrm{~Hz}, 4-\mathrm{H}), 3.82\left(4 \mathrm{H}, \mathrm{s}, 4{ }^{\prime}-\mathrm{OCH}_{3}, \mathrm{~N}-\mathrm{H}\right), 3.73(1 \mathrm{H}, \mathrm{d}, J=10.8 \mathrm{~Hz}, 2-\mathrm{H})$, $2.22-2.11(1 \mathrm{H}, \mathrm{m}, 3-\mathrm{H}), 2.11\left(3 \mathrm{H}, \mathrm{s}, 6-\mathrm{CH}_{3}\right), 0.57\left(3 \mathrm{H}, \mathrm{d}, J=6.5 \mathrm{~Hz}, 3-\mathrm{CH}_{3}\right) ;{ }^{13} \mathrm{C}$ NMR (101 MHz, $\left.\mathrm{CDCl}_{3}\right), \delta(\mathrm{ppm}): 158.3,150.6,147.7,142.2,136.3,130.6,130.4$ (2), 128.9 (2), 127.8, 127.5, 125.8, 123.8 (2), 114.2, 113.9 (2), 63.6, 55.3, 51.5, 42.3, 20.6, 16.4; GC-MS (EI) $\left(t_{R}=16.62 \mathrm{~min}\right), \mathrm{m} / \mathrm{z} 388.1\left(\mathrm{M}^{+}\right) ;$Anal. calcd for $\mathrm{C}_{24} \mathrm{H}_{24} \mathrm{~N}_{2} \mathrm{O}_{3}$ : C, 74.21; H, 6.23; N, 7.21, found: C, 74.14; H, 6.20; N, 7.15.

Cis-4-(4-methoxyphenyl)-3,6-dimethyl-2-(naphthalen-2-yl)-1,2,3,4-

tetrahydroquinoline (4t): light yellow solid (0.21 g, $0.54 \mathrm{mmol}, 59 \%) \mathrm{R}_{f}$ [hexaneEtOAc $15: 1]=0.30 ;$ m.p. $166-170^{\circ} \mathrm{C}$; IR $(\mathrm{KBr}) v_{\max }=3362,2832,2959,1610$, 1505, $1240 \mathrm{~cm}^{-1} ;{ }^{1} \mathrm{H}$ NMR $\left(400 \mathrm{MHz}, \mathrm{CDCl}_{3}\right), \delta(\mathrm{ppm}): 7.91-7.46(7 \mathrm{H}, \mathrm{m}$, AllHArnaft), 7.18 (2H, d, $J=8.6 \mathrm{~Hz}, 3$ 3",5"-HAr), 6.90 (2H, d, $J=8.6 \mathrm{~Hz}, 2$ 2",6"-HAr), $6.84(1 \mathrm{H}, \mathrm{dd}, J=7.8,1.8 \mathrm{~Hz}, 7-\mathrm{H}), 6.50(1 \mathrm{H}, \mathrm{d}, J=8.0 \mathrm{~Hz}, 8-\mathrm{H}), 6.44(1 \mathrm{H}, \mathrm{s}, 5-\mathrm{H})$, $4.26(1 \mathrm{H}, \mathrm{d}, J=9.9 \mathrm{~Hz}, 4-\mathrm{H}), 4.04(1 \mathrm{H}, \mathrm{s}, \mathrm{N}-\mathrm{H}), 3.83\left(3 \mathrm{H}, \mathrm{s}, 4\right.$ "' $\left.-\mathrm{CH}_{3}\right), 3.77(1 \mathrm{H}, \mathrm{d}, J$ $=10.9 \mathrm{~Hz}, 2-\mathrm{H}), 2.37-2.24(1 \mathrm{H}, \mathrm{m}, 3-\mathrm{H}), 2.12\left(3 \mathrm{H}, \mathrm{s}, 6-\mathrm{CH}_{3}\right), 0.59(3 \mathrm{H}, \mathrm{d}, J=6.5$ $\left.\mathrm{Hz}, 3-\mathrm{CH}_{3}\right) ;{ }^{13} \mathrm{C} \mathrm{NMR}\left(101 \mathrm{MHz}, \mathrm{CDCl}_{3}\right), \delta(\mathrm{ppm}): 158.2,142.9,140.4,136.9$, $133.4,133.3,130.6,130.5(2), 128.4,127.9,127.8,127.6,127.2,126.9,126.2$, 126.0, 125.9, 125.6, 113.9, 113.9 (2), 64.3, 55.3, 51.8, 42.0, 20.6, 16.7; GC-MS (EI) $\left(t_{R}=19.076 \mathrm{~min}\right), \mathrm{m} / \mathrm{z} 393.2\left(\mathrm{M}^{+}\right)$; Anal. calcd for $\mathrm{C}_{28} \mathrm{H}_{27} \mathrm{NO}: \mathrm{C}, 85.46 ; \mathrm{H}, 6.92 ; \mathrm{N}$, 3.56, found: C, 85.35; H, 6.84; N, 3.51 .

Cis-4-(4-methoxyphenyl)-3-methyl-2-(naphthalen-2-yl)-6-nitro-1,2,3,4-

tetrahydroquinoline (4u): yellow solid (0.33 g, $0.77 \mathrm{mmol}, 84 \%) \mathrm{R}_{f}$ [hexane-EtOAc $15: 1]=0.16 ;$ m.p. $118-119{ }^{\circ} \mathrm{C} ; \mathrm{IR}(\mathrm{KBr}) v_{\max }=3365,2834,2954,1605,1503$, 
1348, $1239 \mathrm{~cm}^{-1} ;{ }^{1} \mathrm{H}$ NMR (400 MHz, $\left.\mathrm{CDCl}_{3}\right), \delta(\mathrm{ppm}): 7.98-7.44(7 \mathrm{H}, \mathrm{m}$, AllHAr), 7.13 (2H, d, $J=8.7 \mathrm{~Hz}, 3$ ", 5"-HAr), 6.91 (2H, d, $J=8.7 \mathrm{~Hz}, 2$ ", 6"-HAr), 6.46 $(1 \mathrm{H}, \mathrm{d}, J=8.9 \mathrm{~Hz}, 8-\mathrm{H}), 4.94(1 \mathrm{H}, \mathrm{s}, \mathrm{N}-\mathrm{H}), 4.40(1 \mathrm{H}, \mathrm{d}, J=10.0 \mathrm{~Hz}, 4-\mathrm{H}), 3.83(3 \mathrm{H}$, s, 4"- $\left.-\mathrm{OCH}_{3}\right), 3.74(1 \mathrm{H}, \mathrm{d}, J=11.2 \mathrm{~Hz}, 2-\mathrm{H}), 2.27(1 \mathrm{H}, \mathrm{ddd}, J=11.2,10.0,6.4 \mathrm{~Hz}$, 3-H), $0.61\left(3 \mathrm{H}, \mathrm{d}, J=6.5 \mathrm{~Hz}, 3-\mathrm{CH}_{3}\right) ;{ }^{13} \mathrm{C} \mathrm{NMR}\left(101 \mathrm{MHz}, \mathrm{CDCl}_{3}\right), \delta(\mathrm{ppm}): 158.8$, $150.3,138.6,138.2,133.9,133.5,133.4,130.3(2), 129.0,127.9,127.9,127.4,126.7$, $126.6,126.5,125.0,124.9,124.4,114.5(2), 112.4,63.9,55.3,50.8,40.1,16.4$; GCMS (EI) $\left(t_{R}=15.040 \mathrm{~min}\right), \mathrm{m} / \mathrm{z} 424.1\left(\mathrm{M}^{+}\right)$; Anal. calcd for $\mathrm{C}_{27} \mathrm{H}_{24} \mathrm{~N}_{2} \mathrm{O}_{3}$ : C, 76.40; $\mathrm{H}$, 5.70; N, 6.60, found: C, 76.38; H, 5.62; N, 6.52.

Cis-2-(furan-2-yl)-4-(4-methoxyphenyl)-3-methyl-6-nitro-1,2,3,4tetrahydroquinoline (4v): orange solid $(0.27 \mathrm{~g}, 0.74 \mathrm{mmol}, 81 \%) \mathrm{R}_{f}$ [hexane-EtOAc $15: 1]=0.33 ;$ m.p. $170-171^{\circ} \mathrm{C} ; \mathrm{IR}(\mathrm{KBr}) v_{\max }=3326,2836,2955,1601,1505$, 1295, $1239 \mathrm{~cm}^{-1} ;{ }^{1} \mathrm{H}$ NMR (400 MHz, $\left.\mathrm{CDCl}_{3}\right), \delta(\mathrm{ppm}): 7.93-6.81(7 \mathrm{H}, \mathrm{m}$, AllHAr), $6.49-6.35$ (3H, m, 2'-4'-Hfuran), $4.84(1 \mathrm{H}, \mathrm{d}, J=12.8 \mathrm{~Hz}, \mathrm{~N}-\mathrm{H}), 4.38(1 \mathrm{H}$, d, $J=10.2 \mathrm{~Hz}, 4-\mathrm{H}), 3.82(3 \mathrm{H}, \mathrm{s}, 4$ "'-OCH 3$), 3.65(1 \mathrm{H}, \mathrm{d}, J=11.8 \mathrm{~Hz}, 2-\mathrm{H}), 2.34$ $(1 \mathrm{H}, \mathrm{td}, J=11.0,6.4 \mathrm{~Hz}, 3-\mathrm{H}), 0.67\left(3 \mathrm{H}, \mathrm{d}, J=6.6 \mathrm{~Hz}, 3-\mathrm{CH}_{3}\right) ;{ }^{13} \mathrm{C}$ NMR $(101$ $\left.\mathrm{MHz}, \mathrm{CDCl}_{3}\right), \delta(\mathrm{ppm}): 158.8,153.6,149.7,142.6,138.3,133.7,130.2(2), 128.9$ (2), 126.6, 124.3, 114.5, 114.5, 112.5, 110.5, 108.4, 56.8, 55.3, 50.5, 38.0, 16.4; GCMS (EI) $\left(t_{R}=14.796 \mathrm{~min}\right), \mathrm{m} / \mathrm{z}$ 364.1 $\left(\mathrm{M}^{+}\right)$; Anal. calcd for $\mathrm{C}_{21} \mathrm{H}_{20} \mathrm{~N}_{2} \mathrm{O}_{4}: \mathrm{C}, 69.22 ; \mathrm{H}$, 5.53; N, 7.69, found: C, 69.11; H, 5.48; N, 7.60. 
2. Copies of MS, ${ }^{1} \mathrm{H}$ NMR and ${ }^{13} \mathrm{C}$ NMR of THQ compounds.

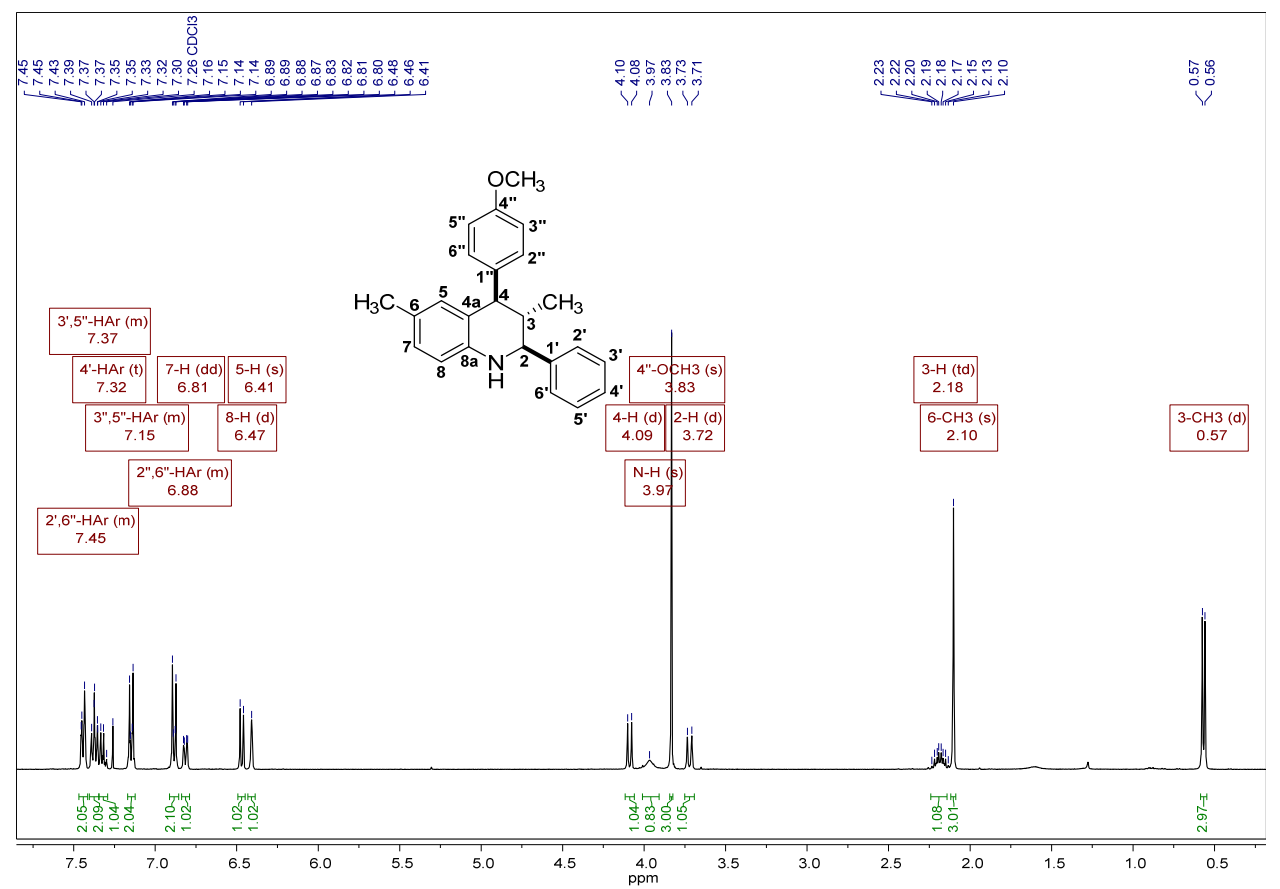

Figure S1. ${ }^{1}$ H-NMR spectra for cis-4-(4-methoxyphenyl)-3,6-dimethyl-2-phenyl-1,2,3,4tetrahydroquinoline (4a)

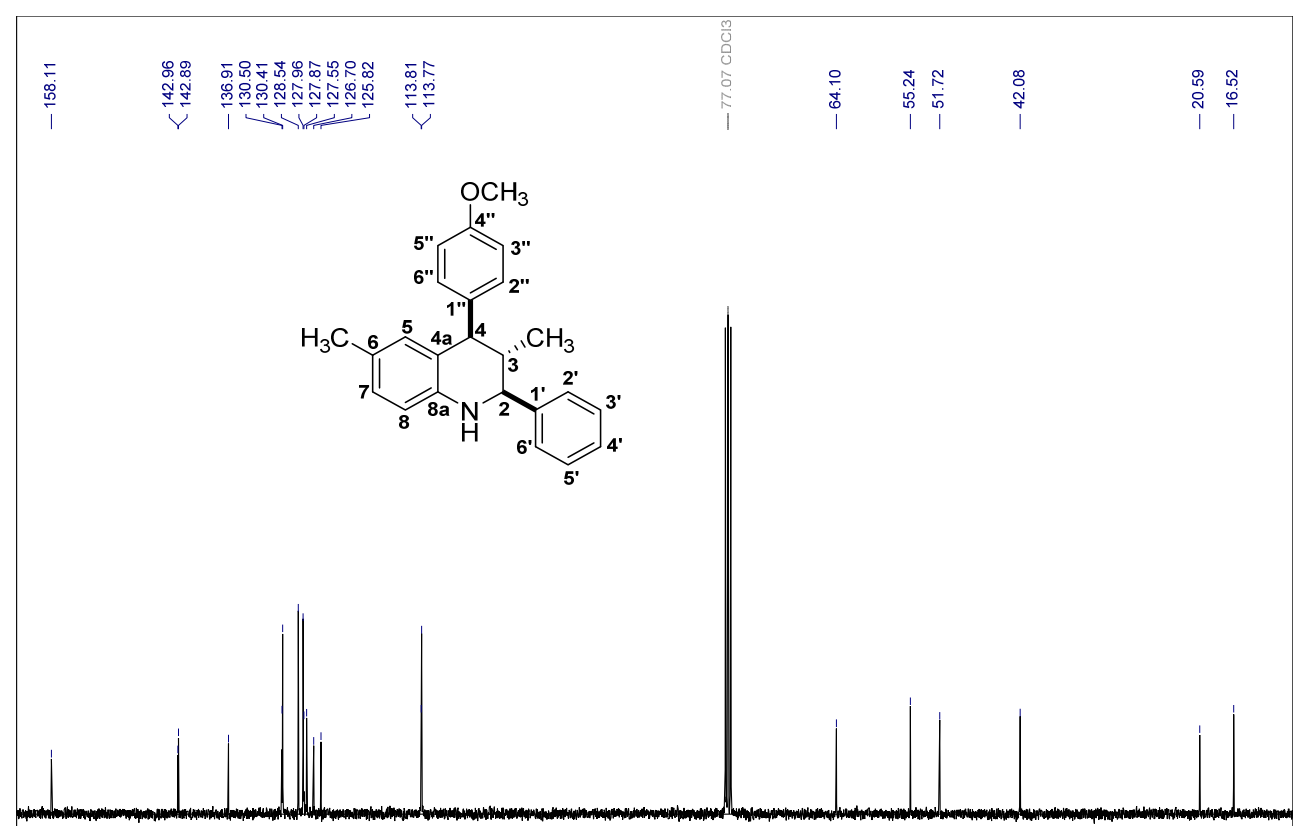

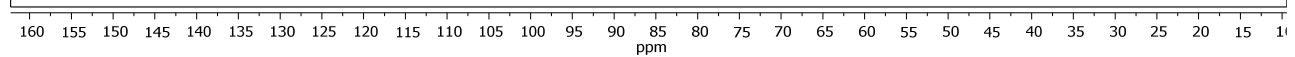

Figure S2. ${ }^{13} \mathrm{C}-\mathrm{NMR}$ spectra for cis-4-(4-methoxyphenyl)-3,6-dimethyl-2-phenyl-1,2,3,4tetrahydroquinoline (4a) 


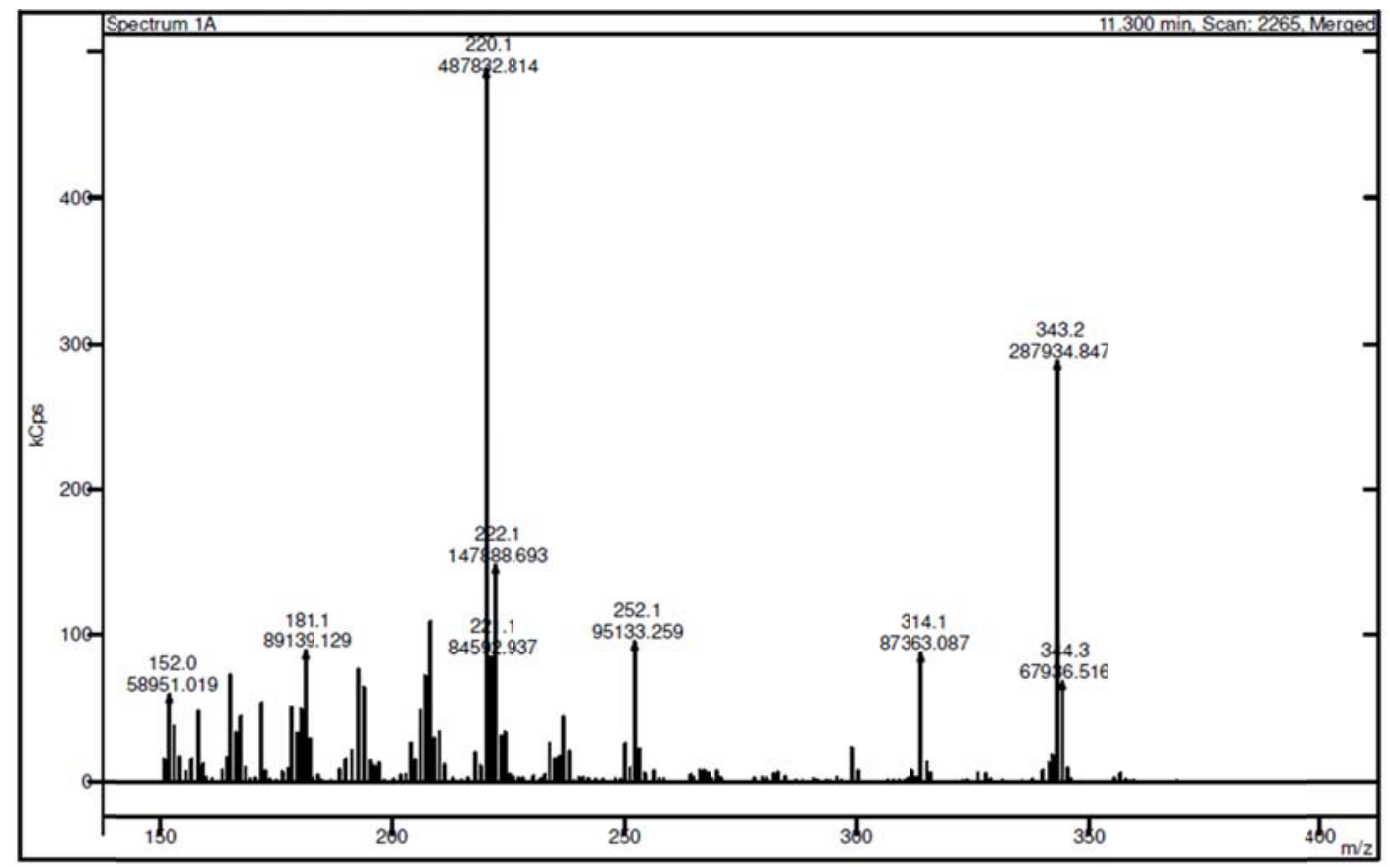

Figure S3. Mass spectra for cis-4-(4-methoxyphenyl)-3,6-dimethyl-2-phenyl-1,2,3,4tetrahydroquinoline (4a)

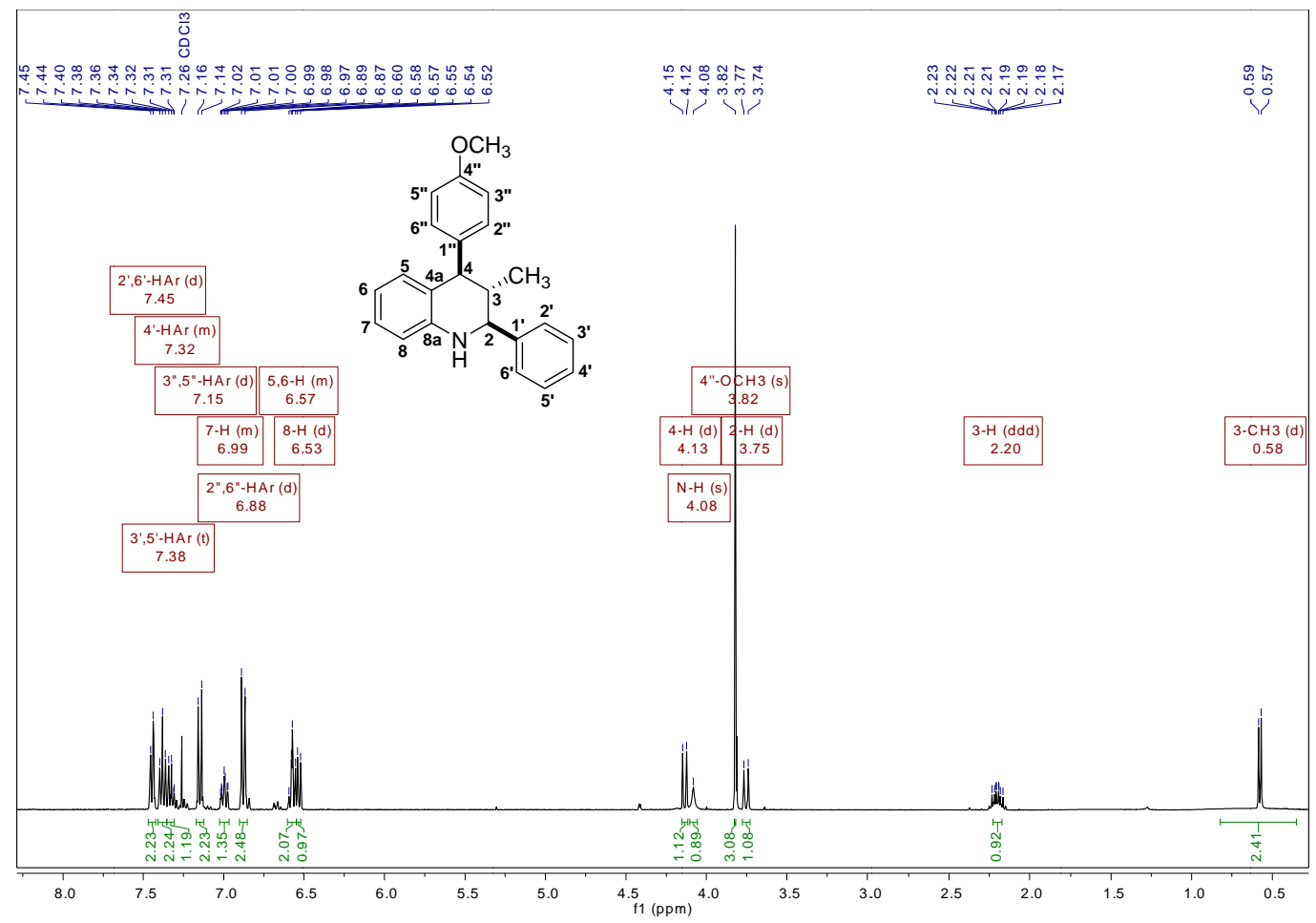

Figure S4. ${ }^{1} \mathrm{H}-\mathrm{NMR}$ spectra for cis-4-(4-methoxyphenyl)-3-methyl-2-phenyl-1,2,3,4tetrahydroquinoline (4b) 


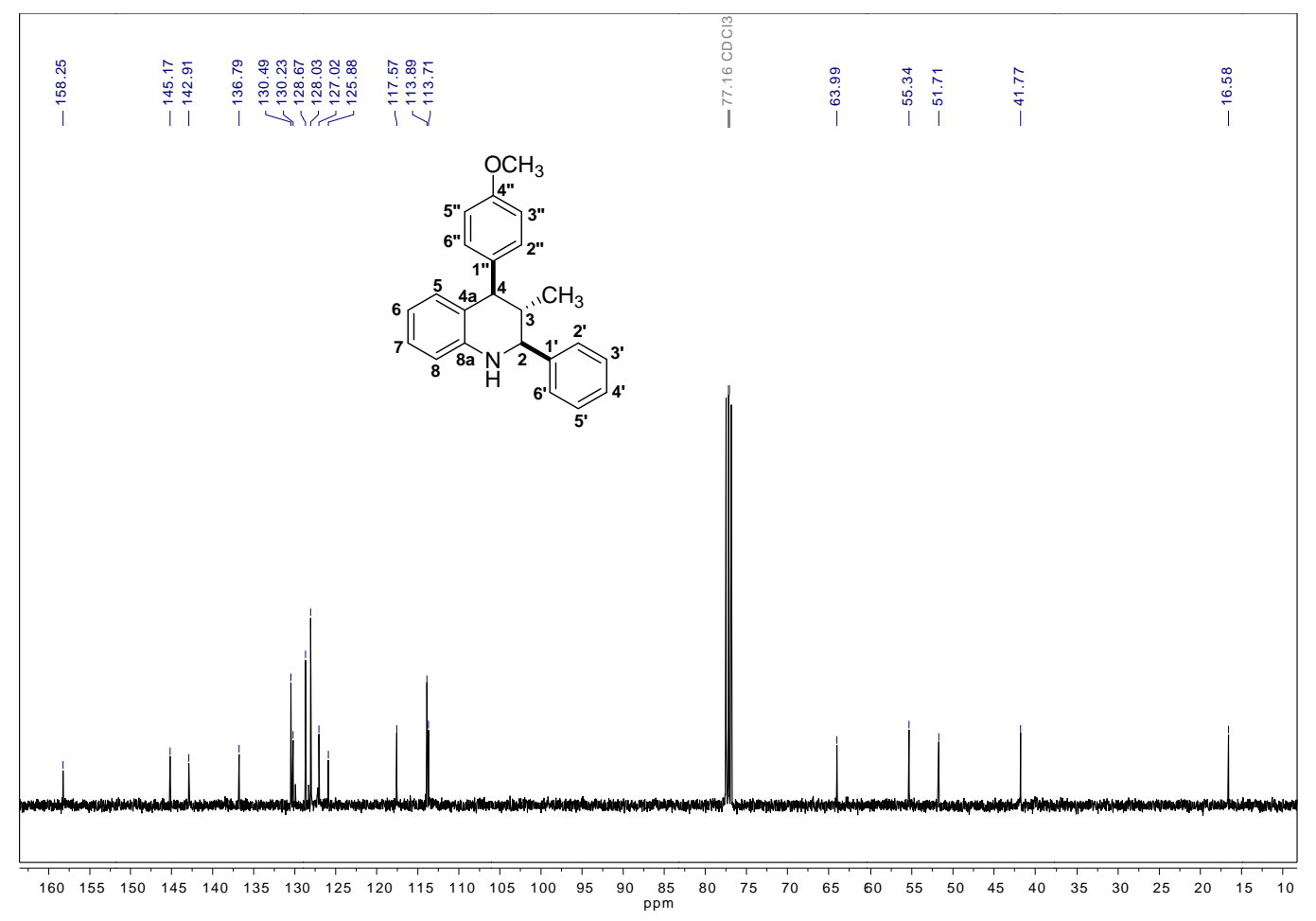

Figure S5. ${ }^{13} \mathrm{C}-\mathrm{NMR}$ spectra for cis-4-(4-methoxyphenyl)-3-methyl-2-phenyl-1,2,3,4tetrahydroquinoline (4b)

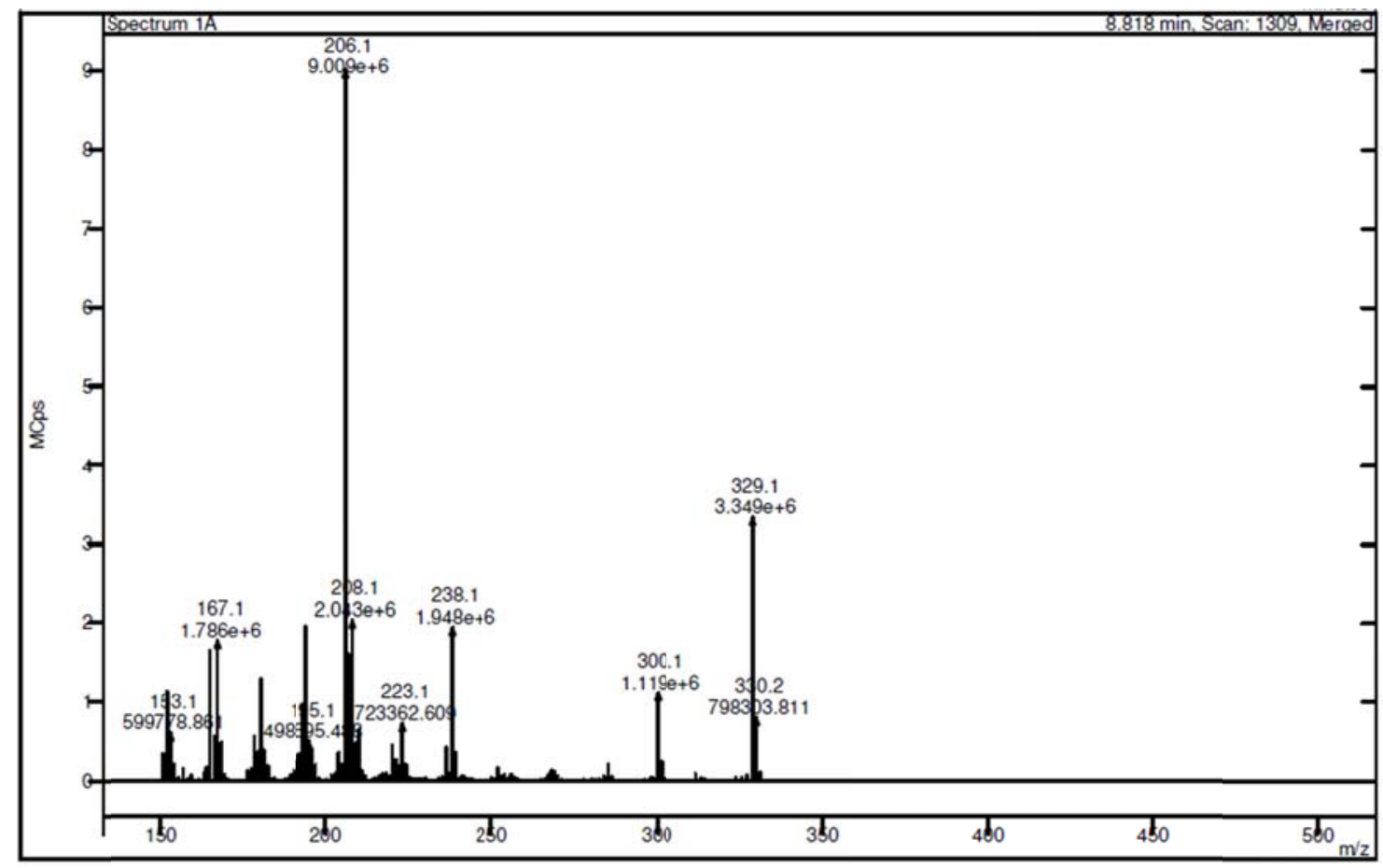

Figure S6. Mass spectra for cis-4-(4-methoxyphenyl)-3-methyl-2-phenyl-1,2,3,4tetrahydroquinoline (4b) 


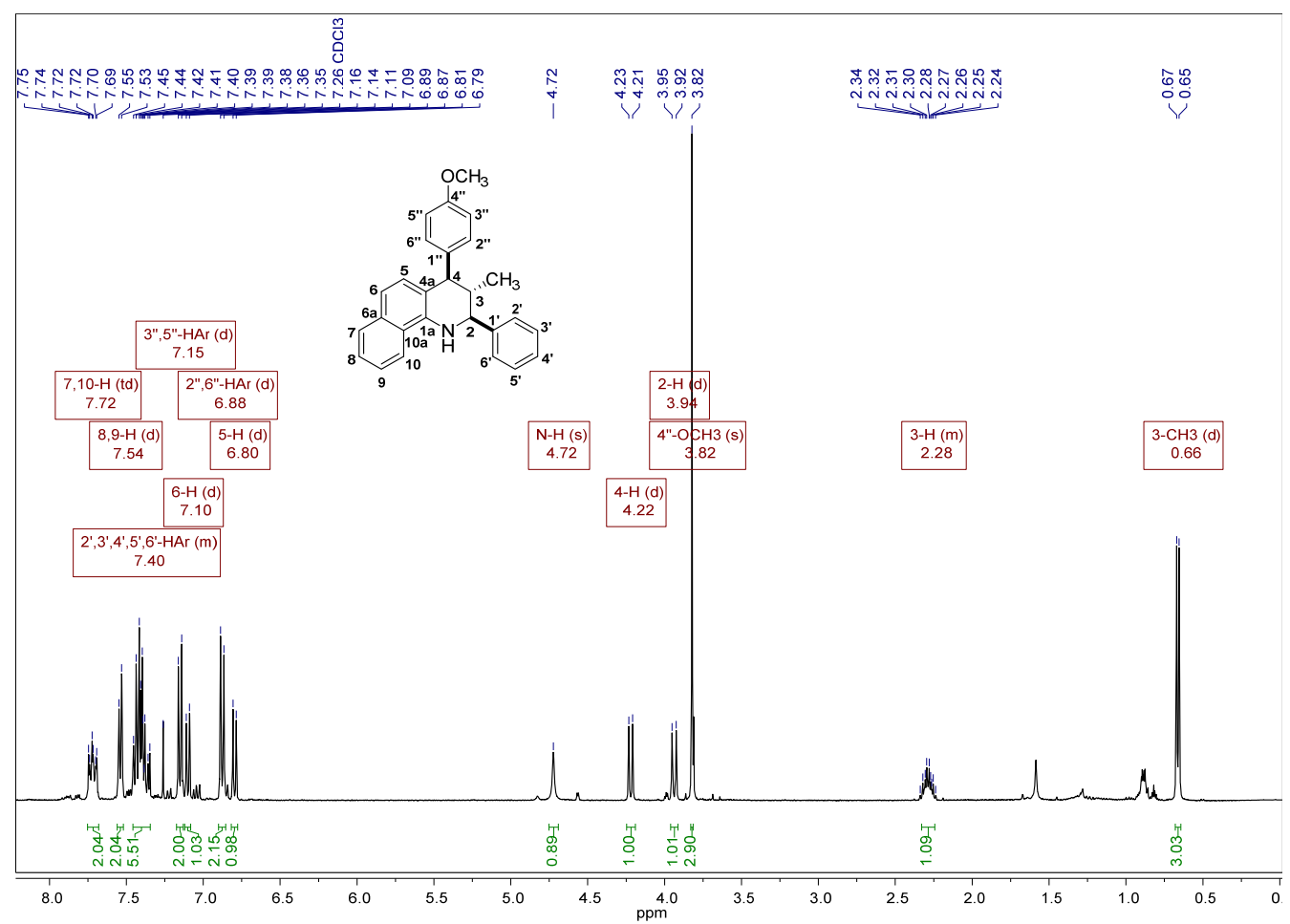

Figure S7. ${ }^{1} \mathrm{H}-\mathrm{NMR}$ spectra for cis-4-(4-methoxyphenyl)-3-methyl-2-phenyl-1,2,3,4tetrahydrobenzo[h]quinoline (4c)

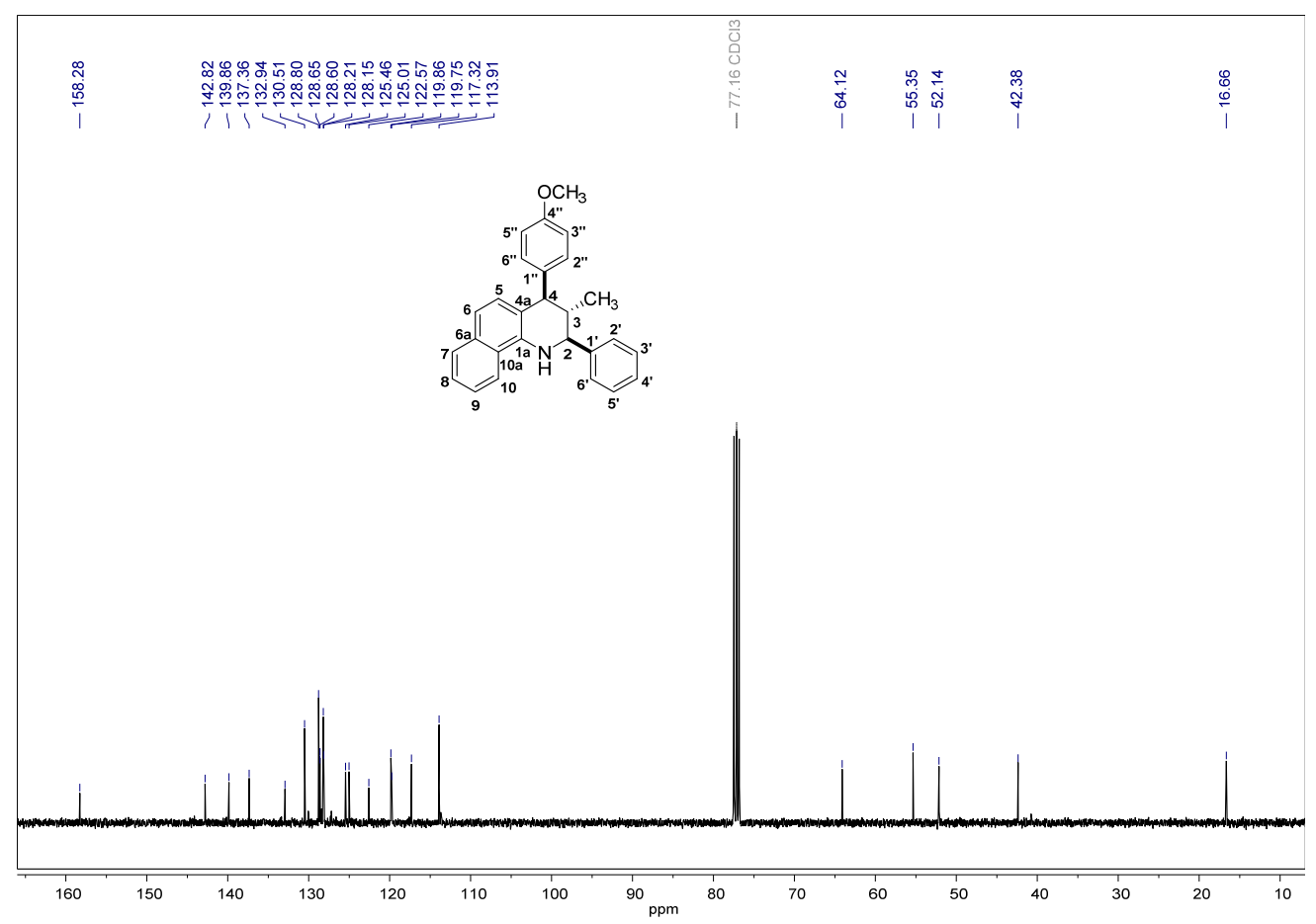

Figure S8. ${ }^{13} \mathrm{C}-\mathrm{NMR}$ spectra for cis-4-(4-methoxyphenyl)-3-methyl-2-phenyl-1,2,3,4tetrahydrobenzo[h]quinoline (4c) 


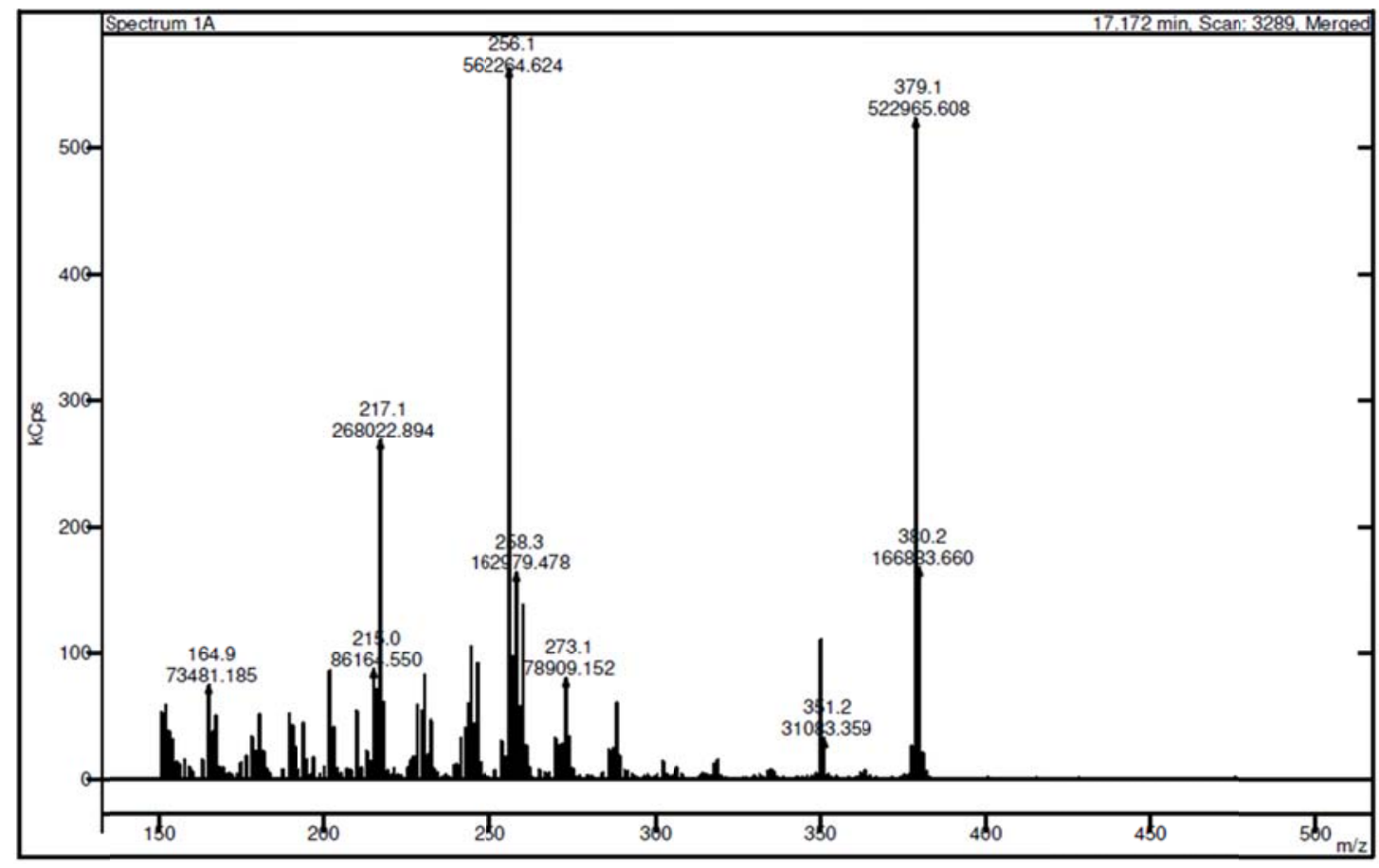

Figure S9. Mass spectra for cis-4-(4-methoxyphenyl)-3-methyl-2-phenyl-1,2,3,4tetrahydrobenzo[h]quinoline (4c)

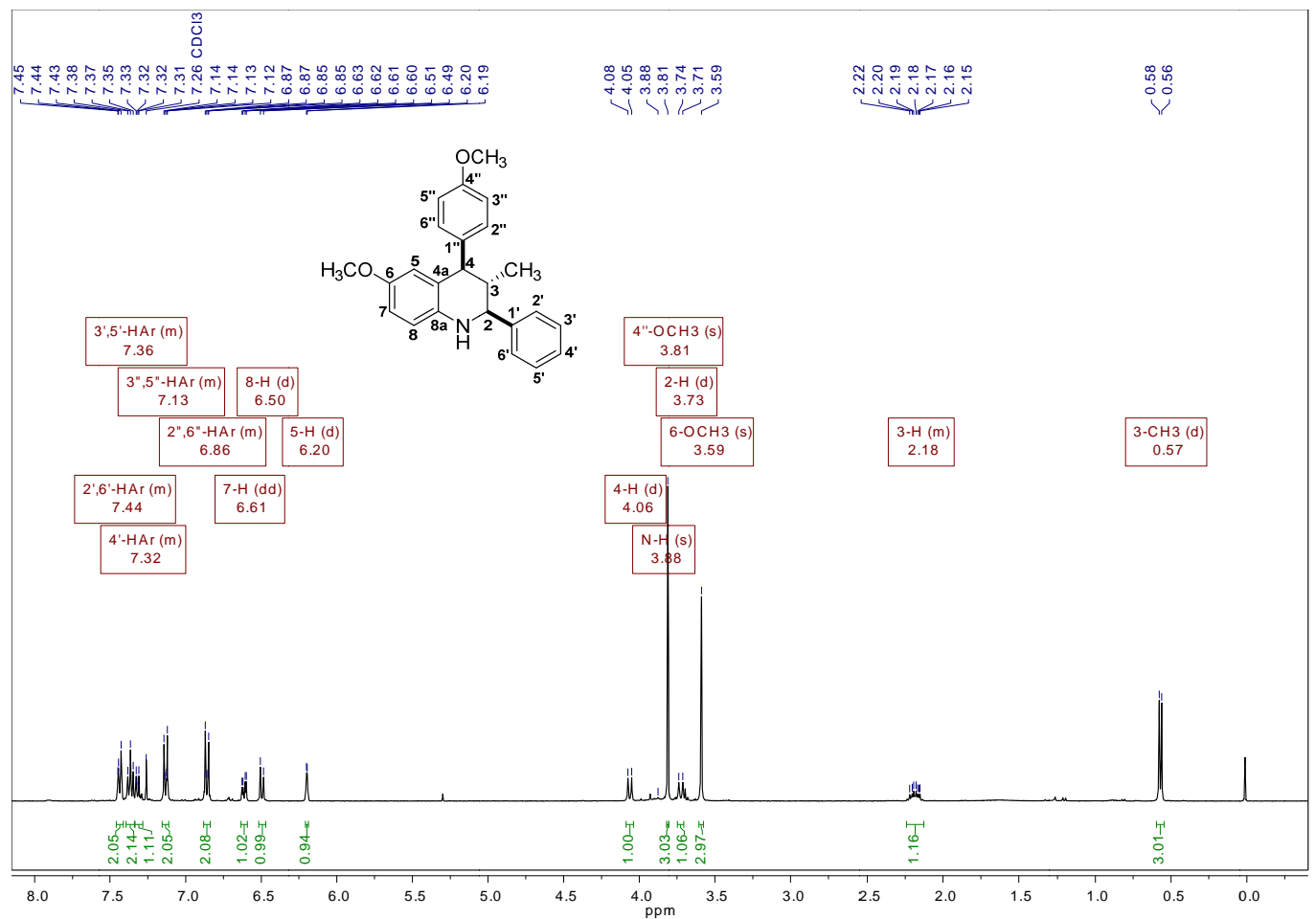

Figure S10. ${ }^{1}$ H-NMR spectra for cis-6-methoxy-4-(4-methoxyphenyl)-3-methyl-2-phenyl1,2,3,4-tetrahydroquinoline (4d) 


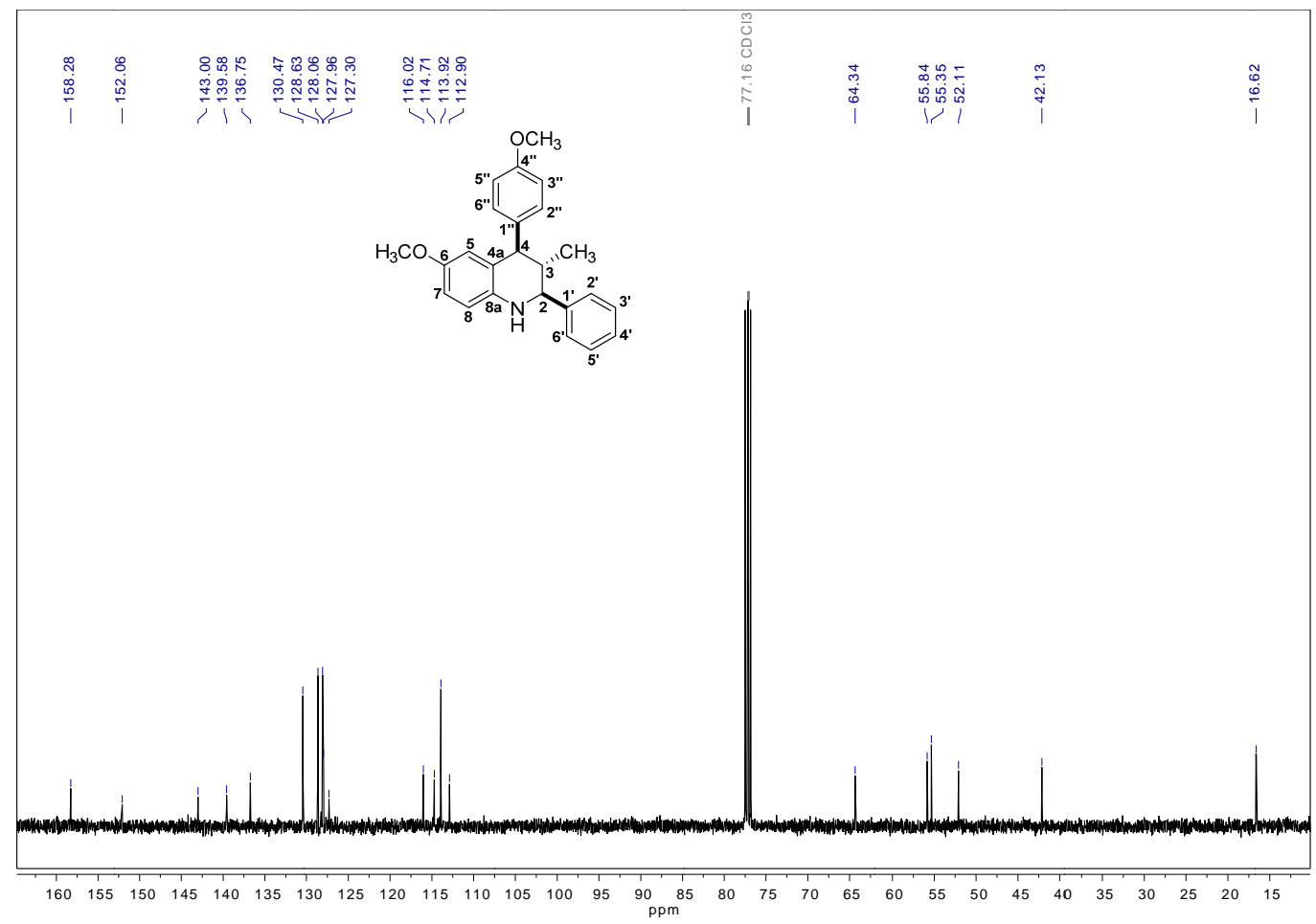

Figure S11. ${ }^{13} \mathrm{C}$-NMR spectra for cis-6-methoxy-4-(4-methoxyphenyl)-3-methyl-2-phenyl1,2,3,4-tetrahydroquinoline (4d)

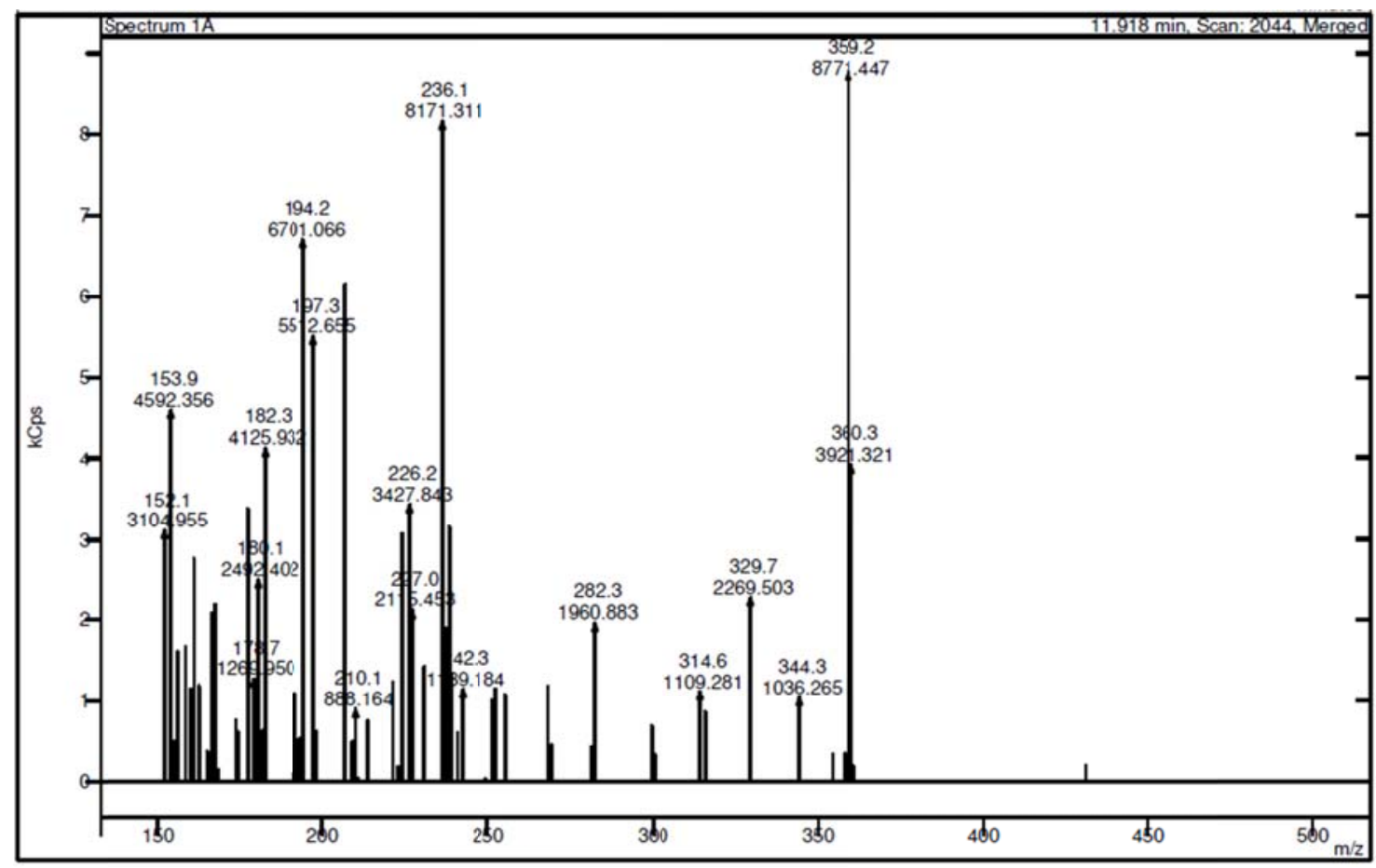

Figure S12. Mass spectra for cis-6-methoxy-4-(4-methoxyphenyl)-3-methyl-2-phenyl1,2,3,4-tetrahydroquinoline (4d) 


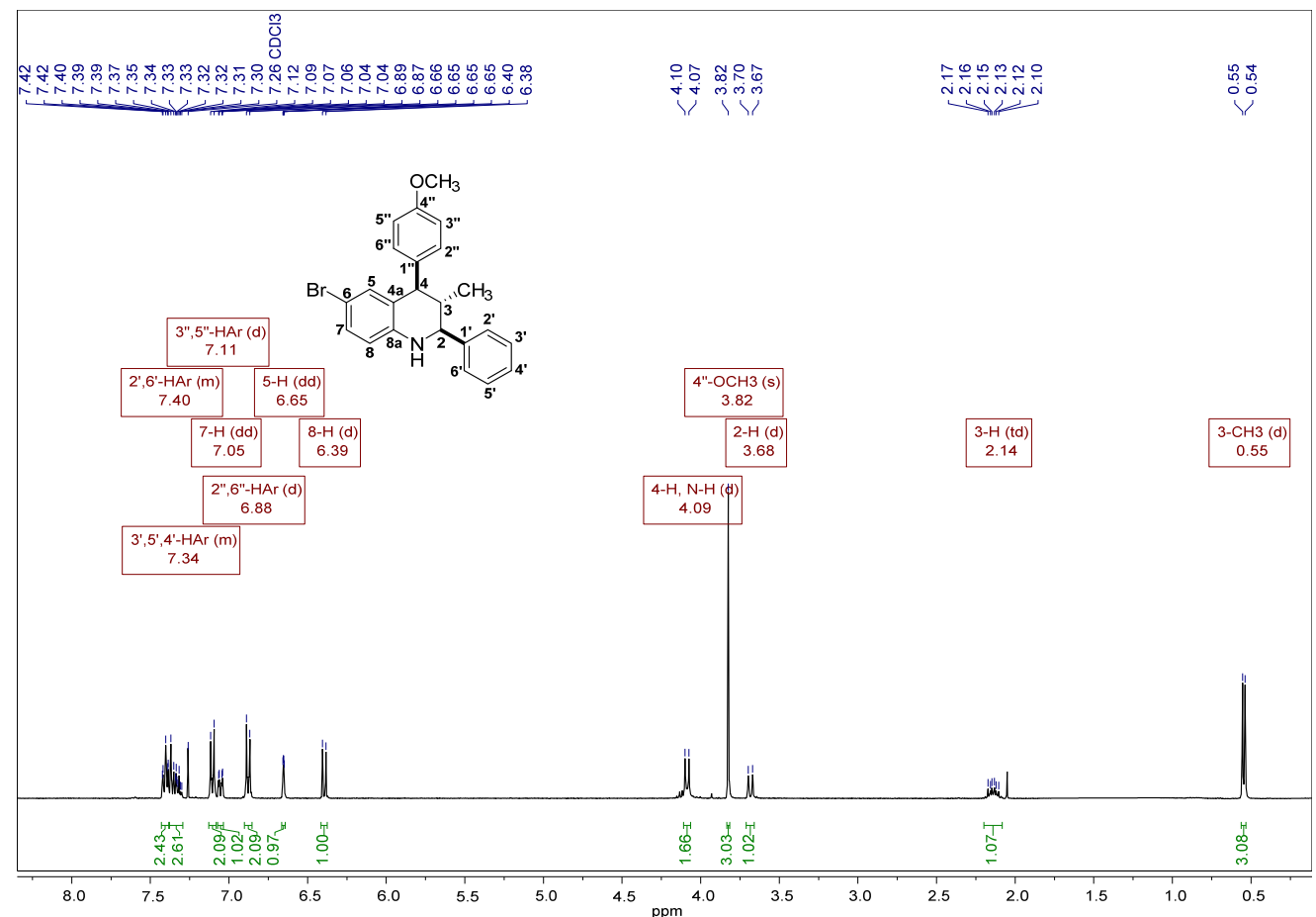

Figure S13. ${ }^{1} \mathrm{H}-\mathrm{NMR}$ spectra for cis-6-bromo-4-(4-methoxyphenyl)-3-methyl-2-phenyl1,2,3,4-tetrahydroquinoline (4e)

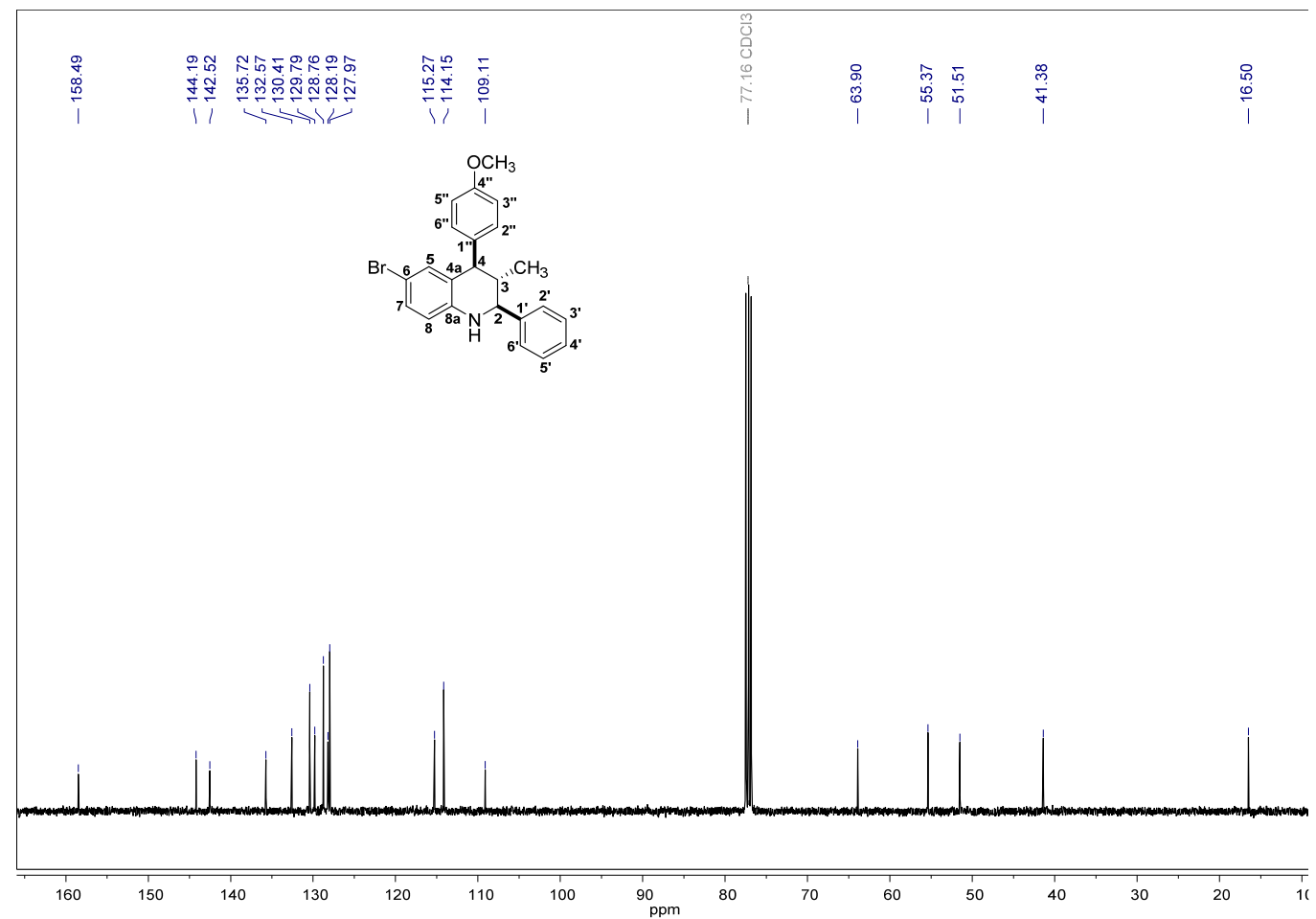

Figure S14. ${ }^{13} \mathrm{C}$-NMR spectra for cis-6-bromo-4-(4-methoxyphenyl)-3-methyl-2-phenyl1,2,3,4-tetrahydroquinoline (4e) 


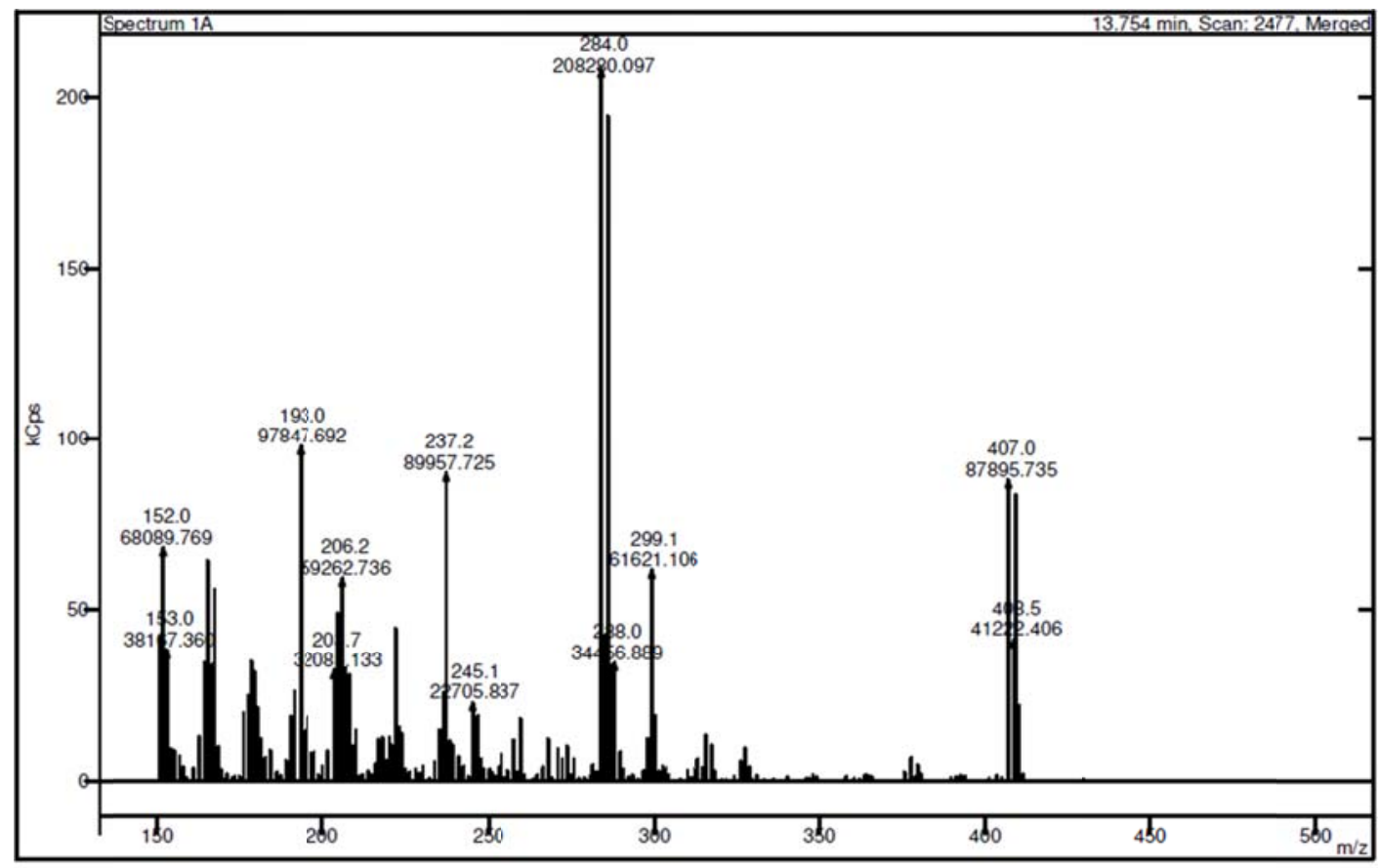

Figure S15. Mass spectra for cis-6-bromo-4-(4-methoxyphenyl)-3-methyl-2-phenyl1,2,3,4-tetrahydroquinoline (4e)

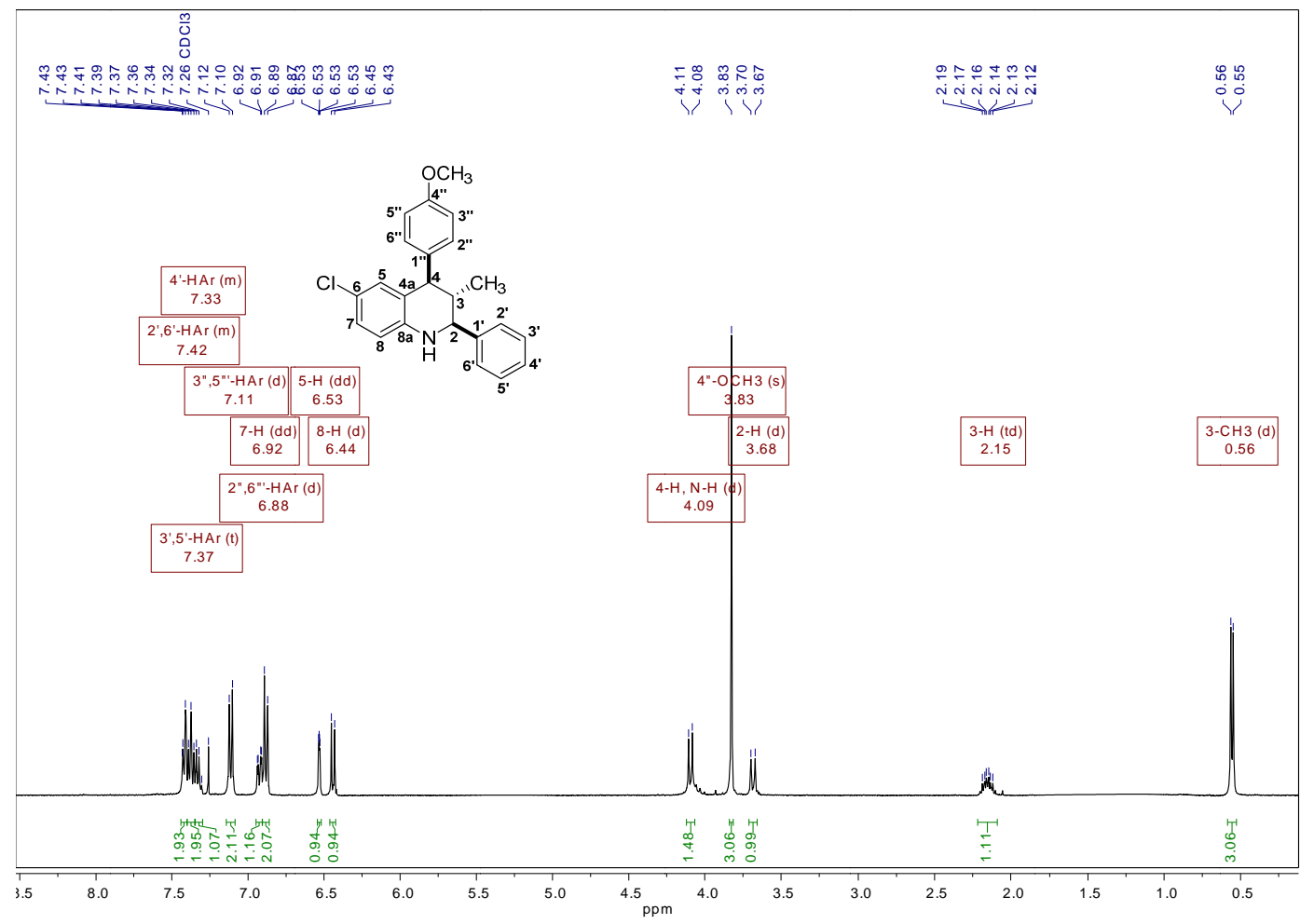

Figure S16. ${ }^{1}$ H-NMR spectra for cis-6-chloro-4-(4-methoxyphenyl)-3-methyl-2-phenyl1,2,3,4-tetrahydroquinoline (4f) 


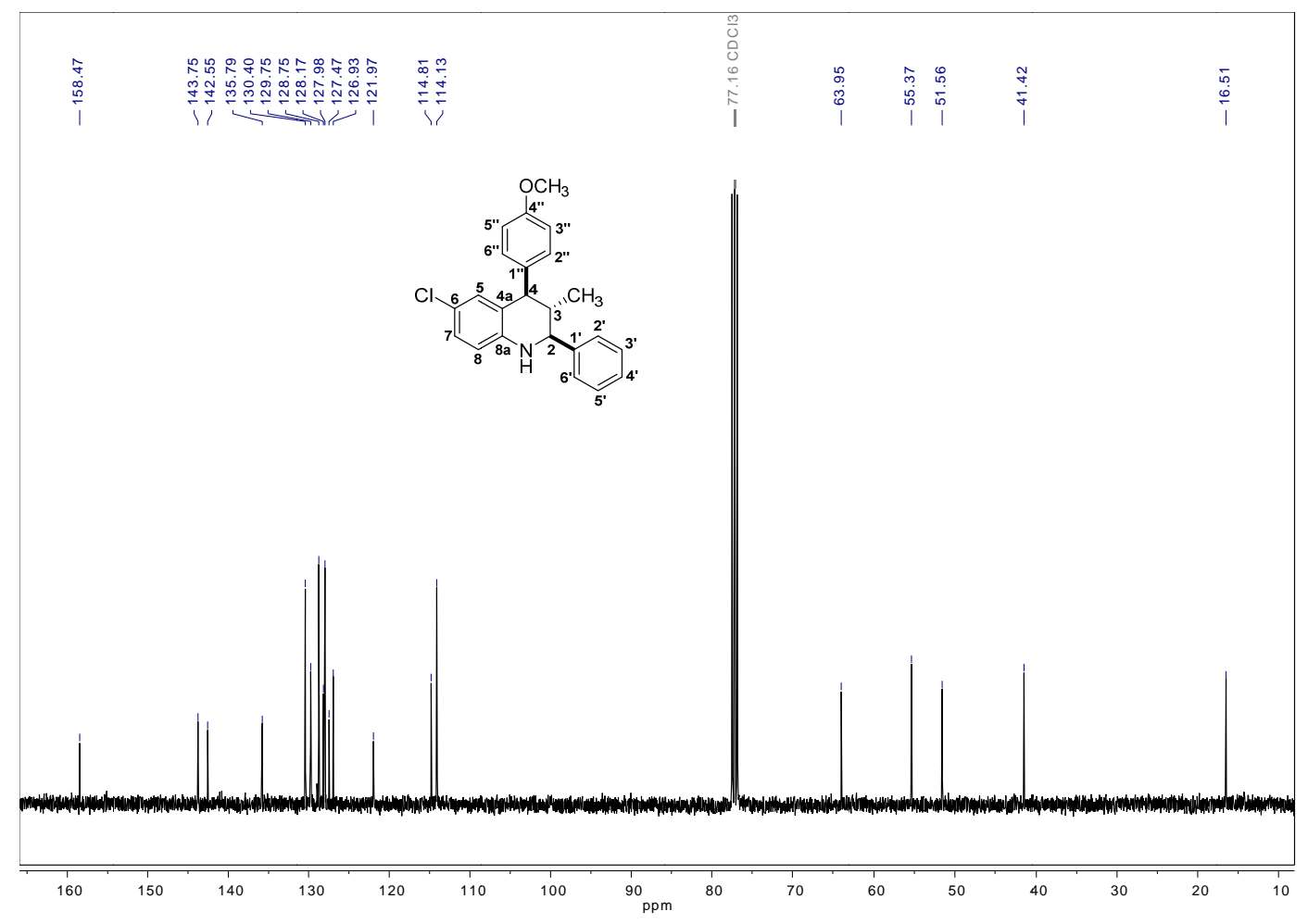

Figure S17. ${ }^{13} \mathrm{C}-\mathrm{NMR}$ spectra for cis-6-chloro-4-(4-methoxyphenyl)-3-methyl-2-phenyl1,2,3,4-tetrahydroquinoline (4f)

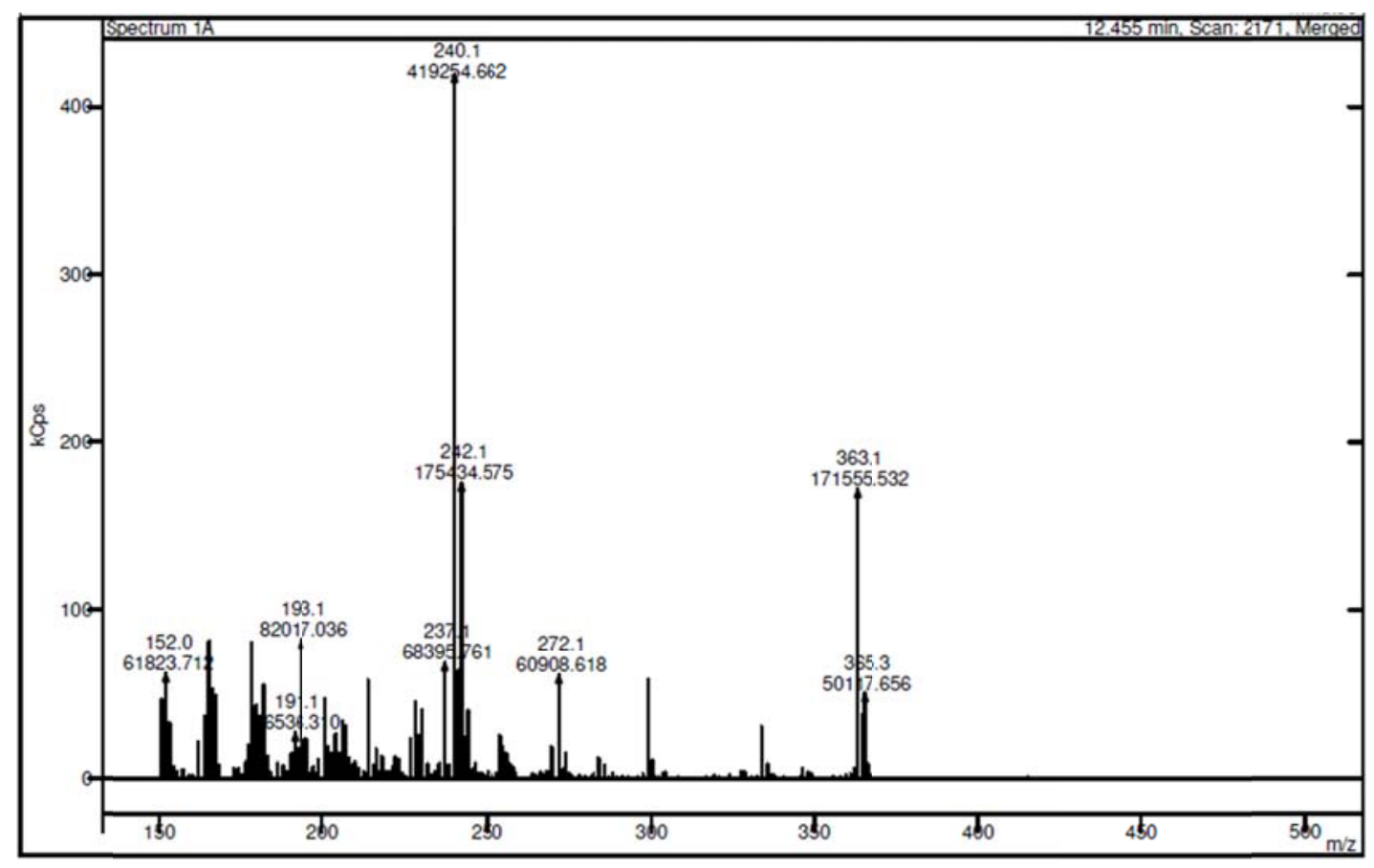

Figure S186. Mass spectra for cis-6-chloro-4-(4-methoxyphenyl)-3-methyl-2-phenyl1,2,3,4-tetrahydroquinoline (4f) 


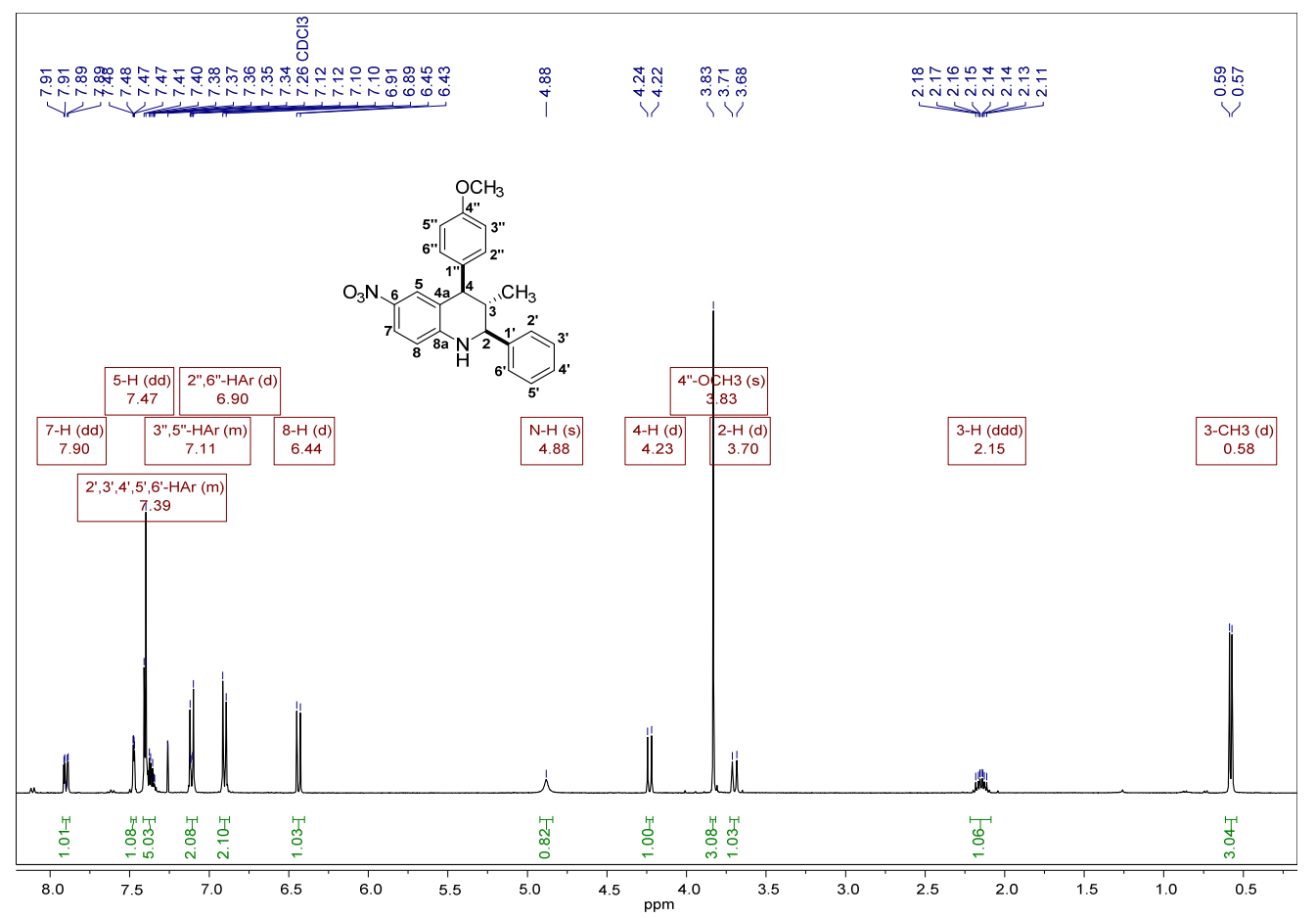

Figure S19. ${ }^{1}$ H-NMR spectra for cis-4-(4-methoxyphenyl)-3-methyl-6-nitro-2-phenyl1,2,3,4-tetrahydroquinoline (4g)

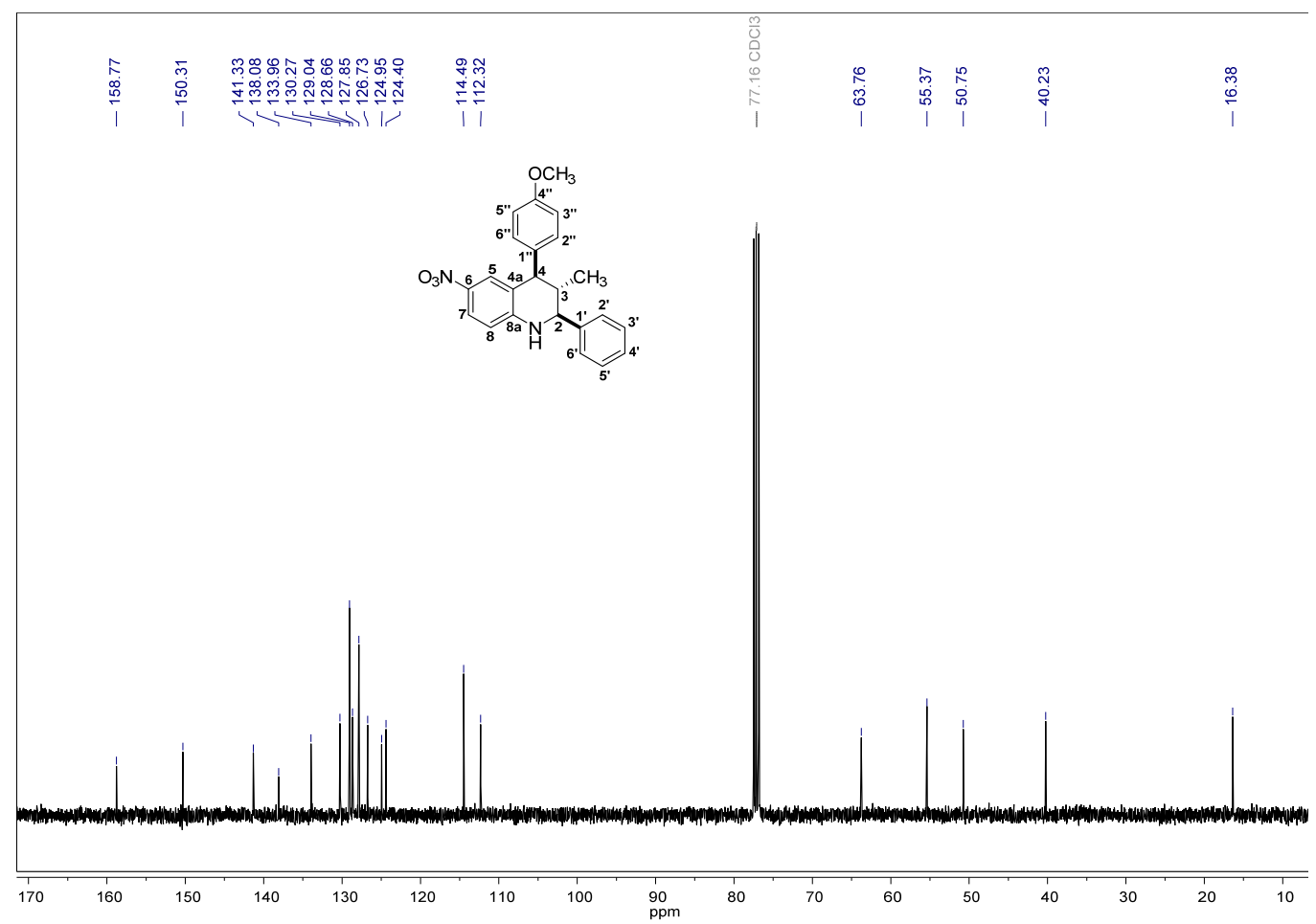

Figure S20. ${ }^{13}$ C-NMR spectra for cis-4-(4-methoxyphenyl)-3-methyl-6-nitro-2-phenyl1,2,3,4-tetrahydroquinoline (4g) 


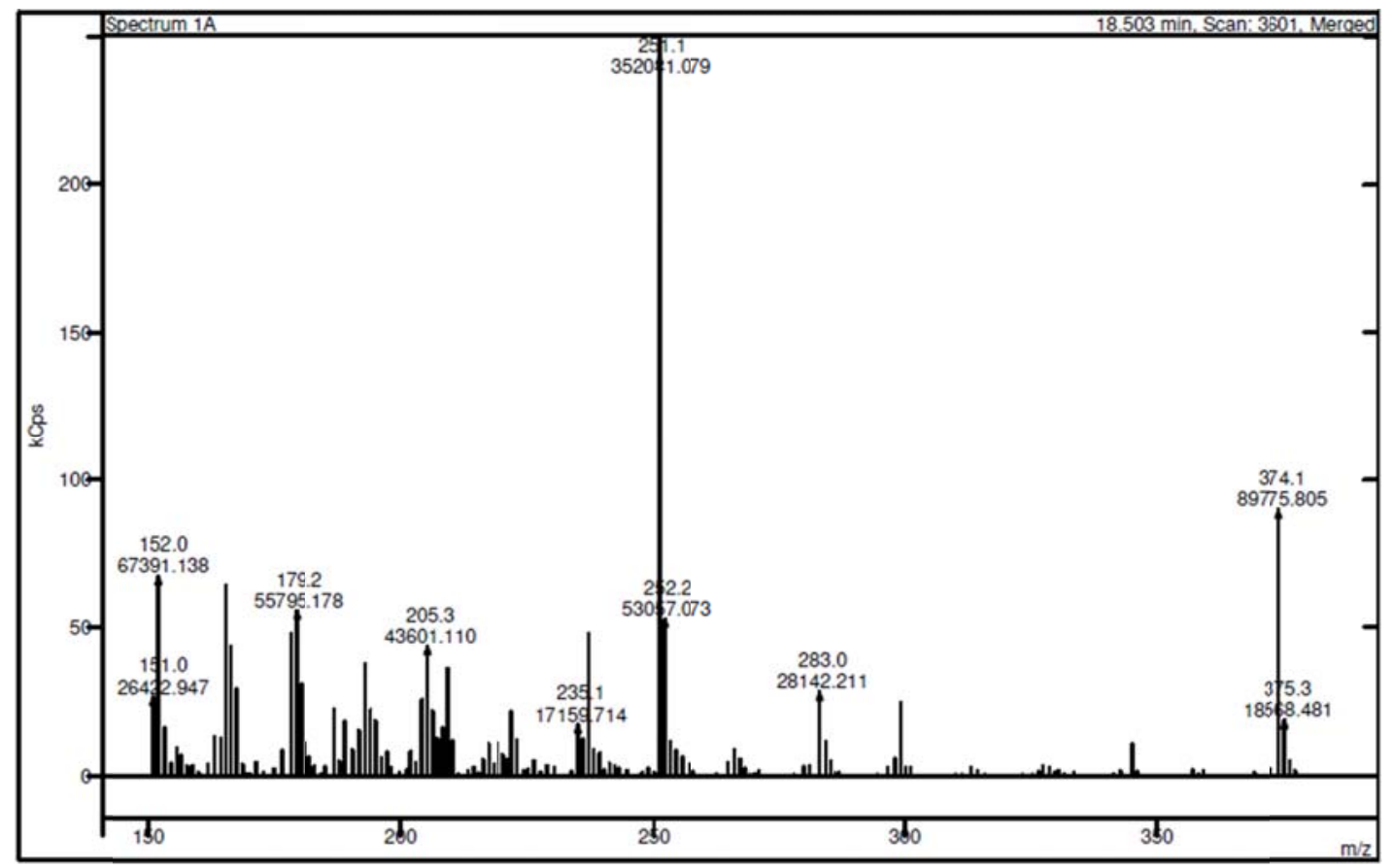

Figure S21. Mass spectra for cis-4-(4-methoxyphenyl)-3-methyl-6-nitro-2-phenyl-1,2,3,4tetrahydroquinoline (4g)

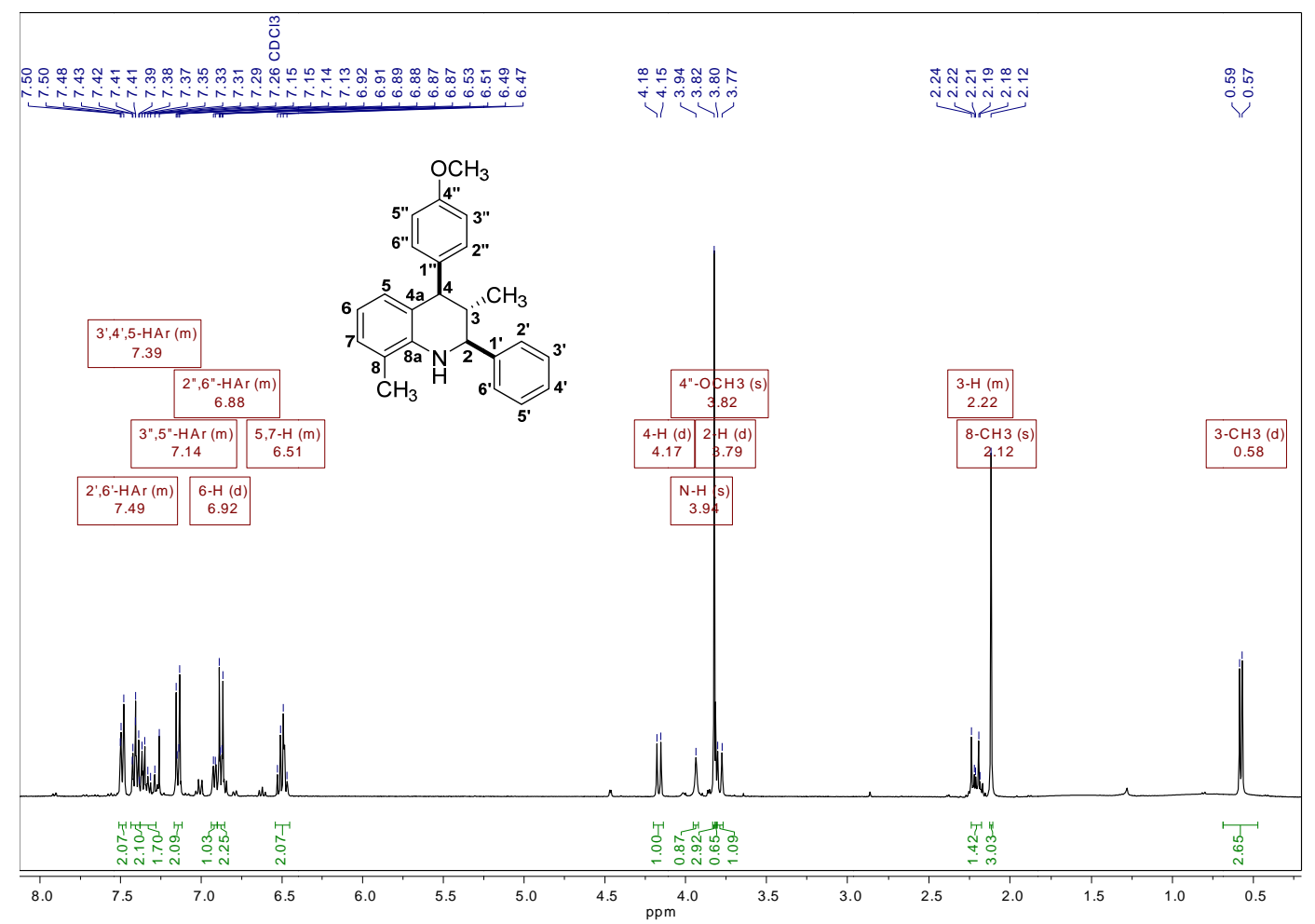

Figure S22. ${ }^{1} \mathrm{H}-\mathrm{NMR}$ spectra for cis-4-(4-methoxyphenyl)-3,8-dimethyl-2-phenyl-1,2,3,4tetrahydroquinoline (4h) 


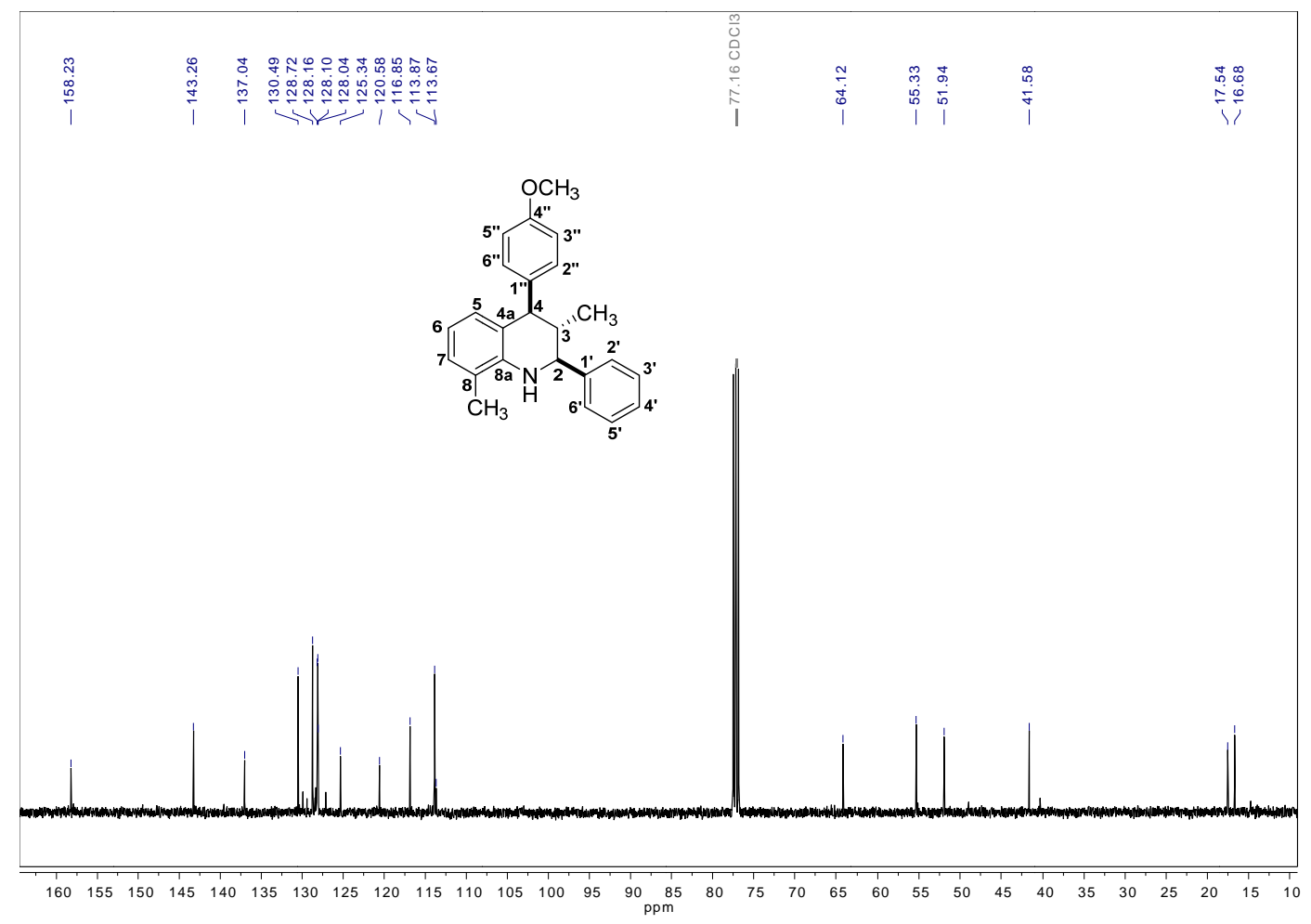

Figure S23. ${ }^{13} \mathrm{C}-\mathrm{NMR}$ spectra for cis-4-(4-methoxyphenyl)-3,8-dimethyl-2-phenyl-1,2,3,4tetrahydroquinoline (4h)

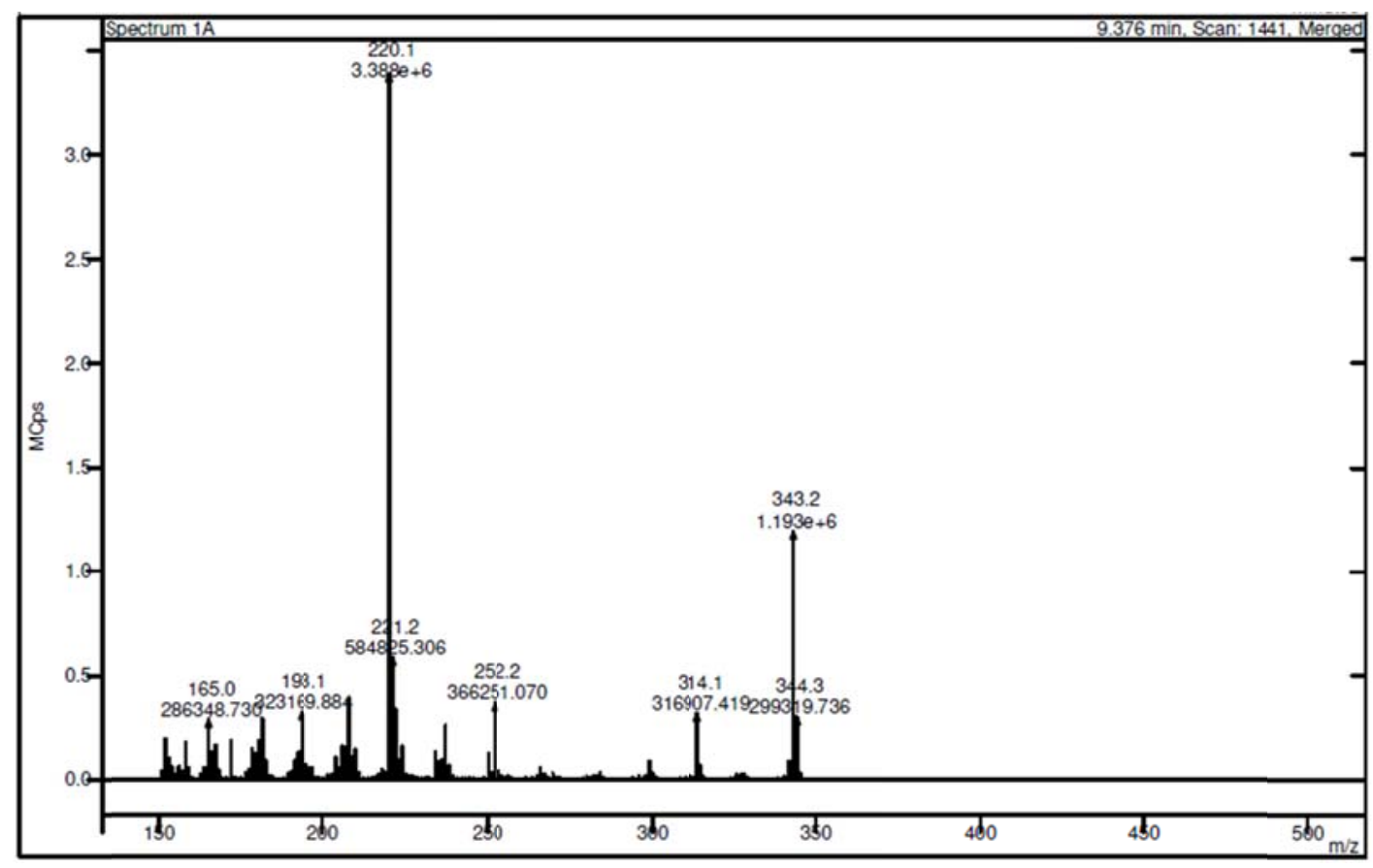

Figure S24. Mass spectra for cis-4-(4-methoxyphenyl)-3,8-dimethyl-2-phenyl-1,2,3,4tetrahydroquinoline (4h) 


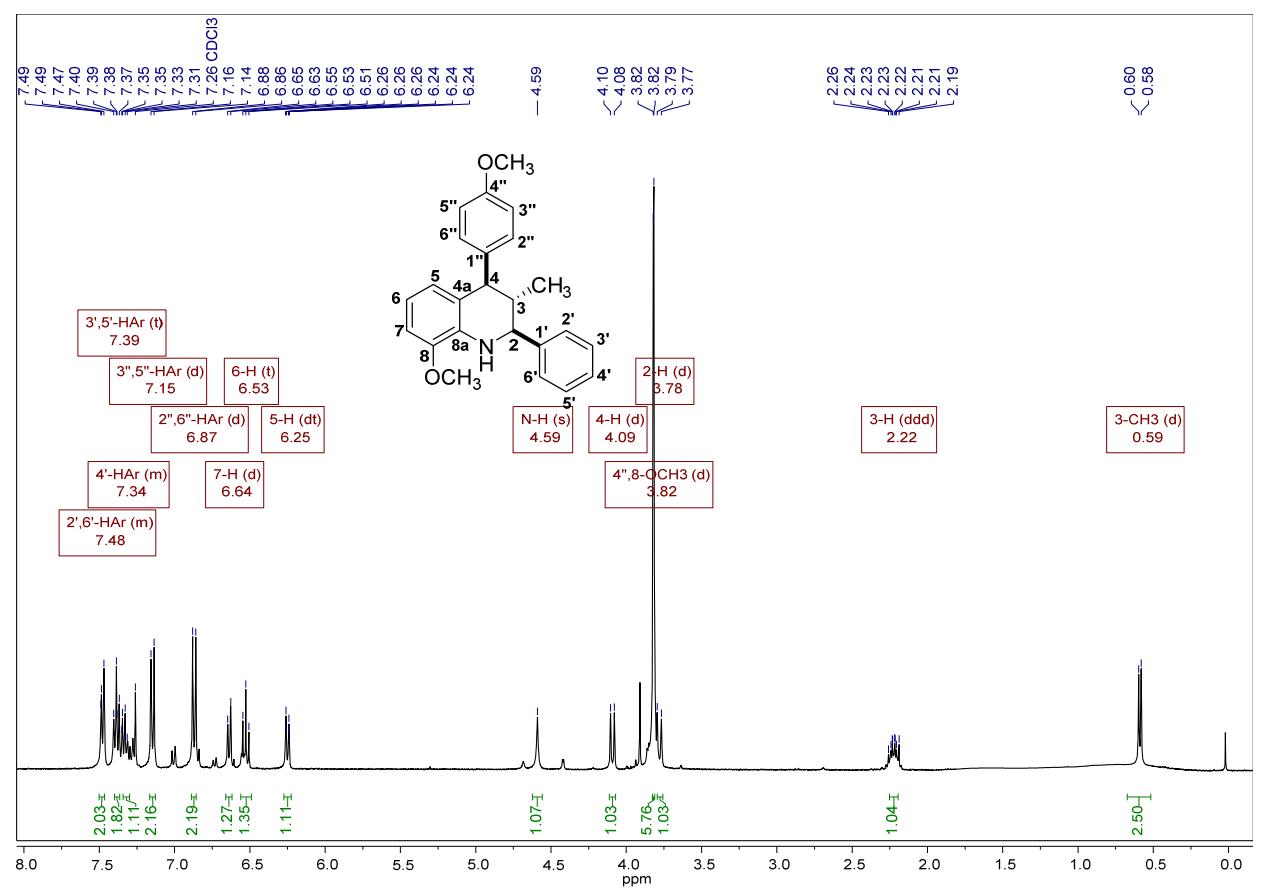

Figure S25. ${ }^{1} \mathrm{H}-\mathrm{NMR}$ spectra for cis-8-methoxy-4-(4-methoxyphenyl)-3-methyl-2-phenyl1,2,3,4-tetrahydroquinoline (4i)

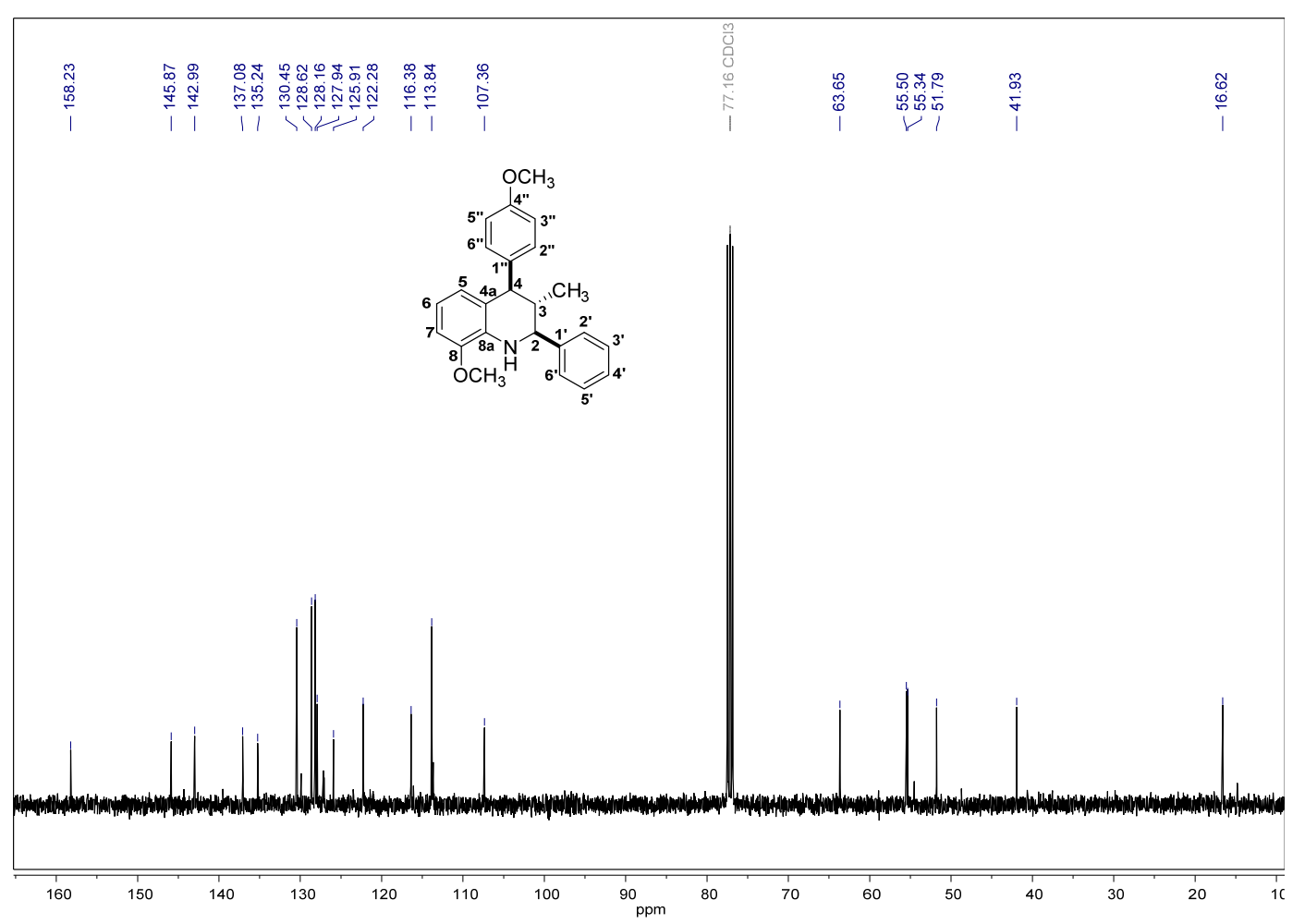

Figure S26. ${ }^{13}$ C-NMR spectra for cis-8-methoxy-4-(4-methoxyphenyl)-3-methyl-2-phenyl1,2,3,4-tetrahydroquinoline (4i) 


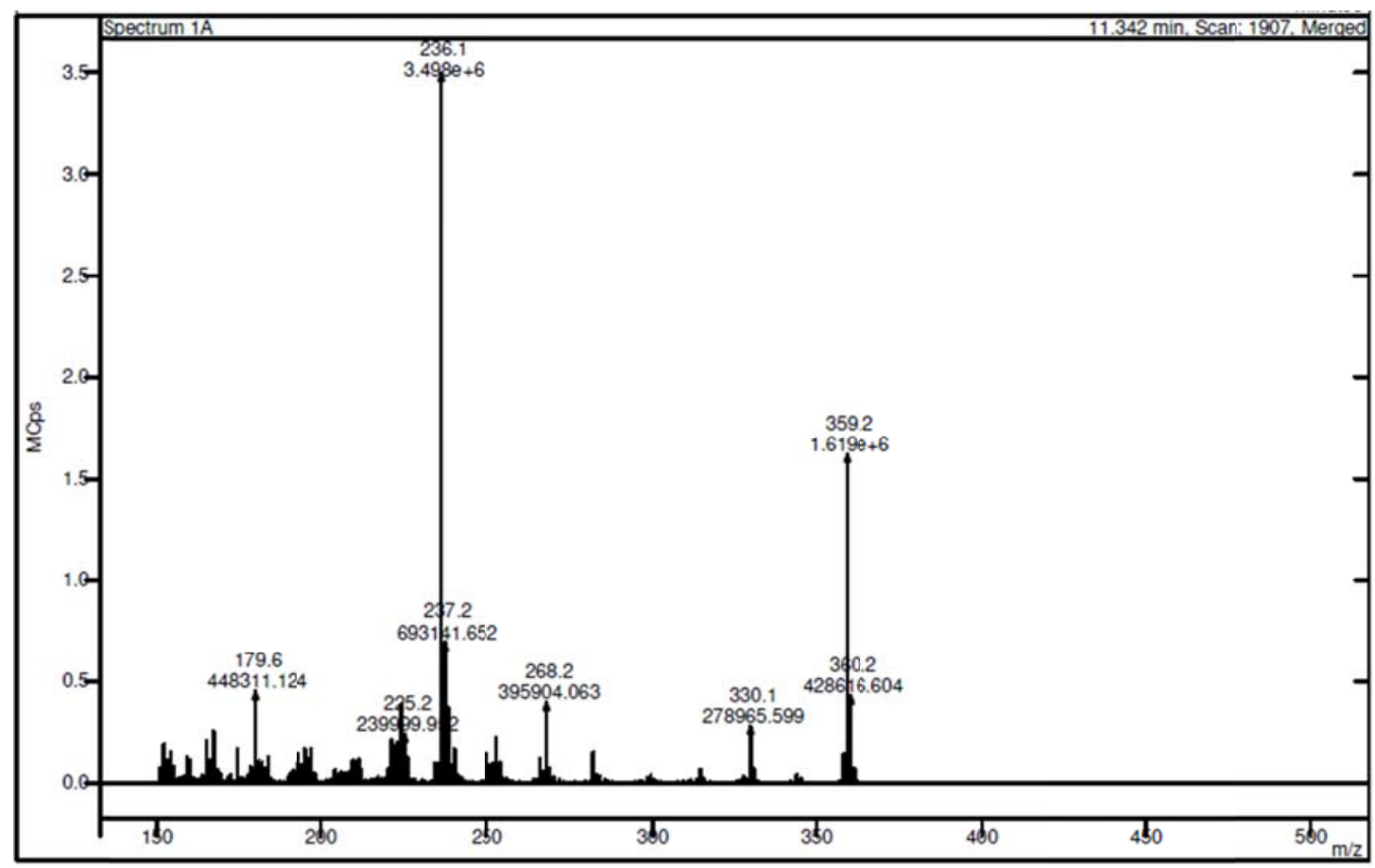

Figure S27. Mass spectra for cis-8-methoxy-4-(4-methoxyphenyl)-3-methyl-2-phenyl1,2,3,4-tetrahydroquinoline (4i)

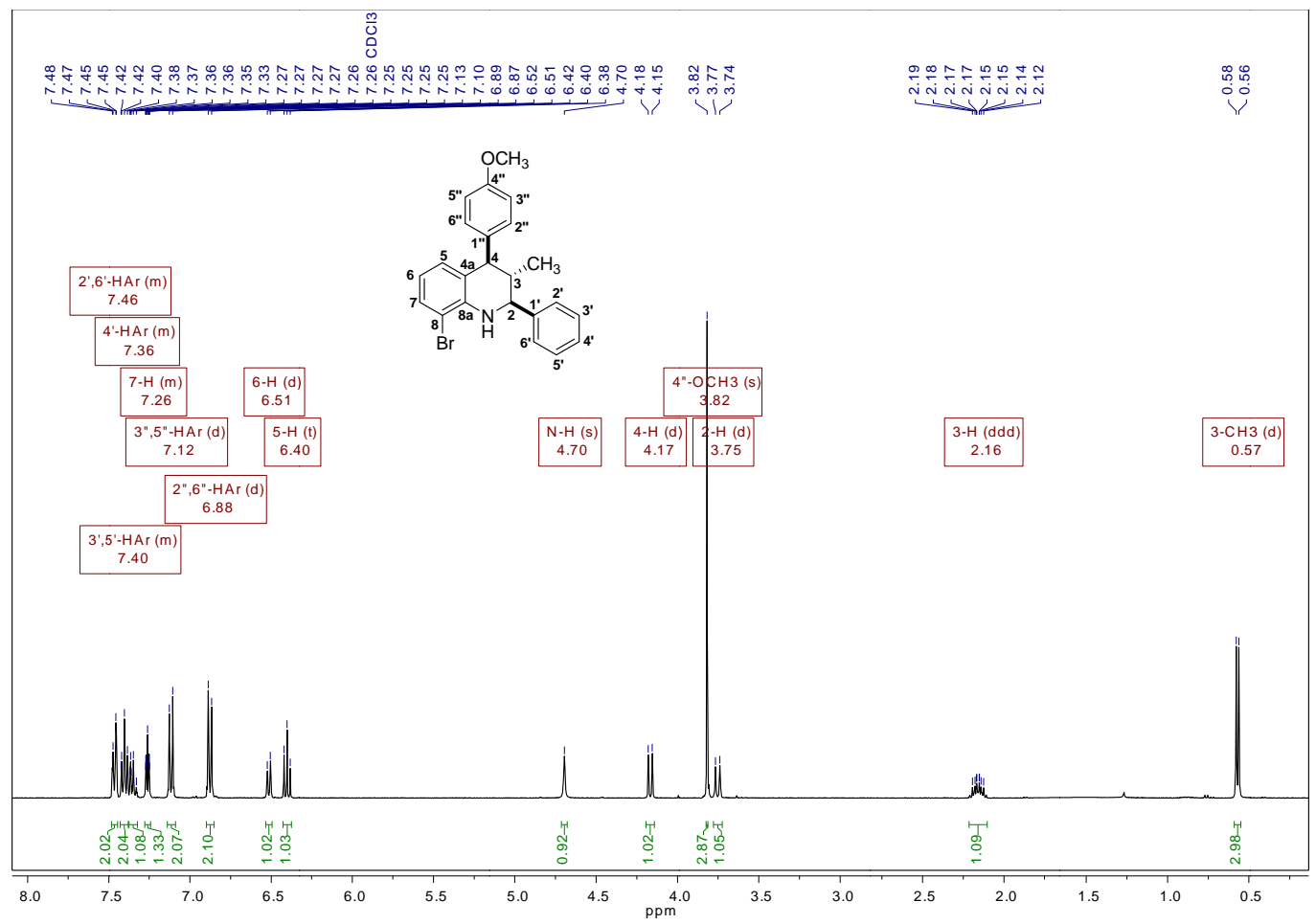

Figure S28. ${ }^{1} \mathrm{H}-\mathrm{NMR}$ spectra for cis-8-bromo-4-(4-methoxyphenyl)-3-methyl-2-phenyl1,2,3,4-tetrahydroquinoline $\mathbf{( 4 \mathbf { j } )}$ 


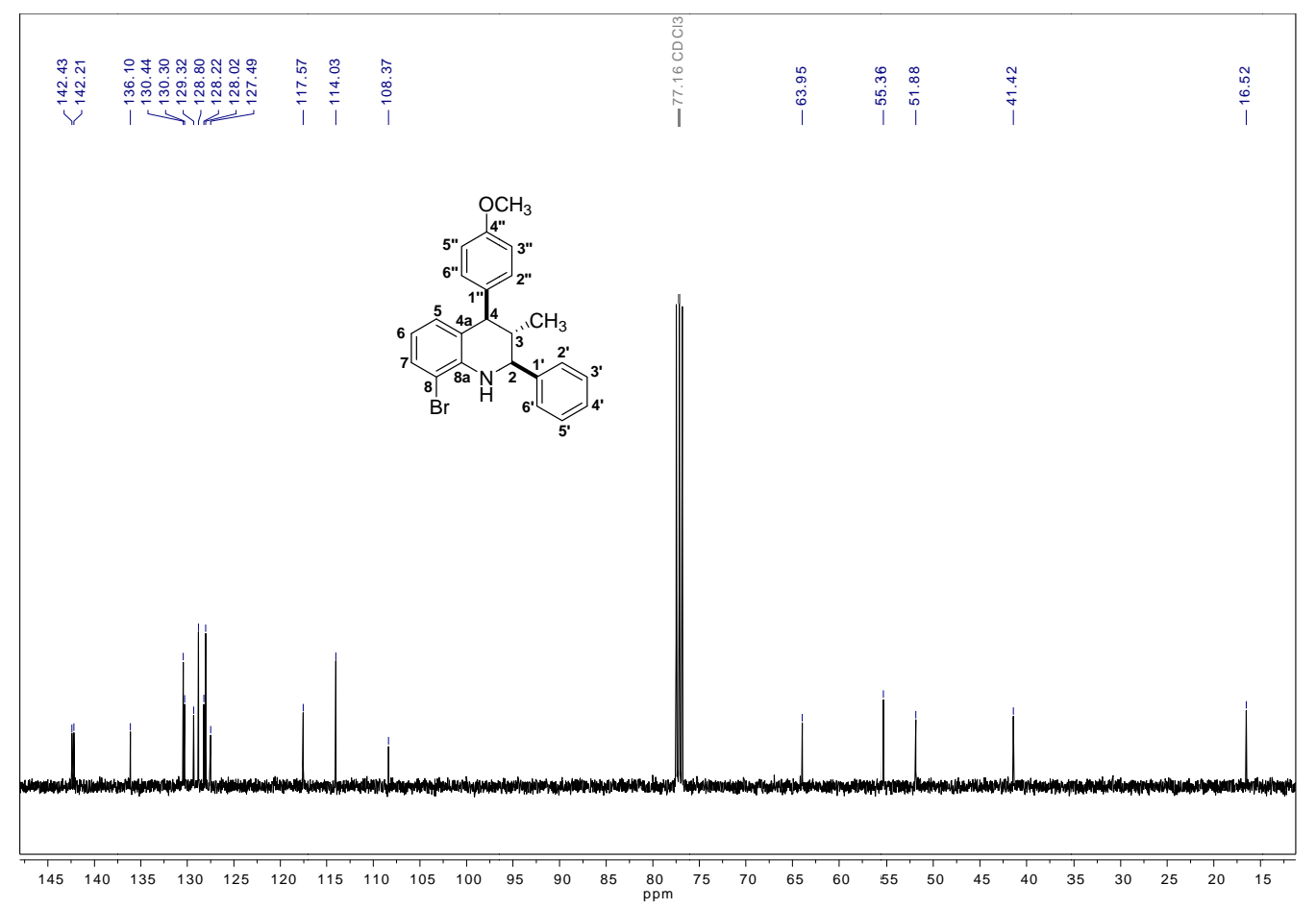

Figure S29. ${ }^{13}$ C-NMR spectra for cis-8-bromo-4-(4-methoxyphenyl)-3-methyl-2-phenyl1,2,3,4-tetrahydroquinoline $\mathbf{( 4 \mathbf { j } )}$

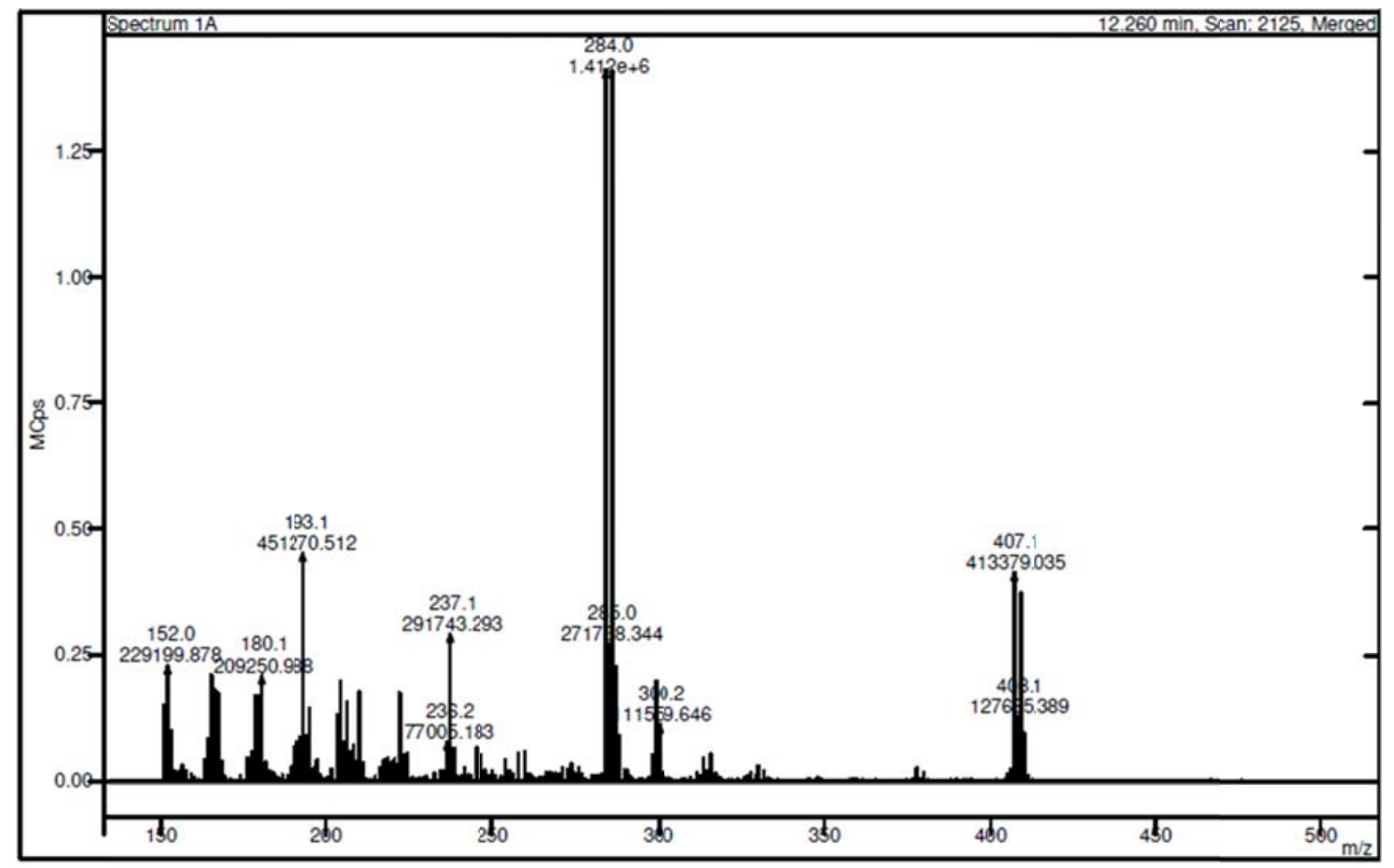

Figure S30. Mass spectra for cis-8-bromo-4-(4-methoxyphenyl)-3-methyl-2-phenyl1,2,3,4-tetrahydroquinoline (4j) 


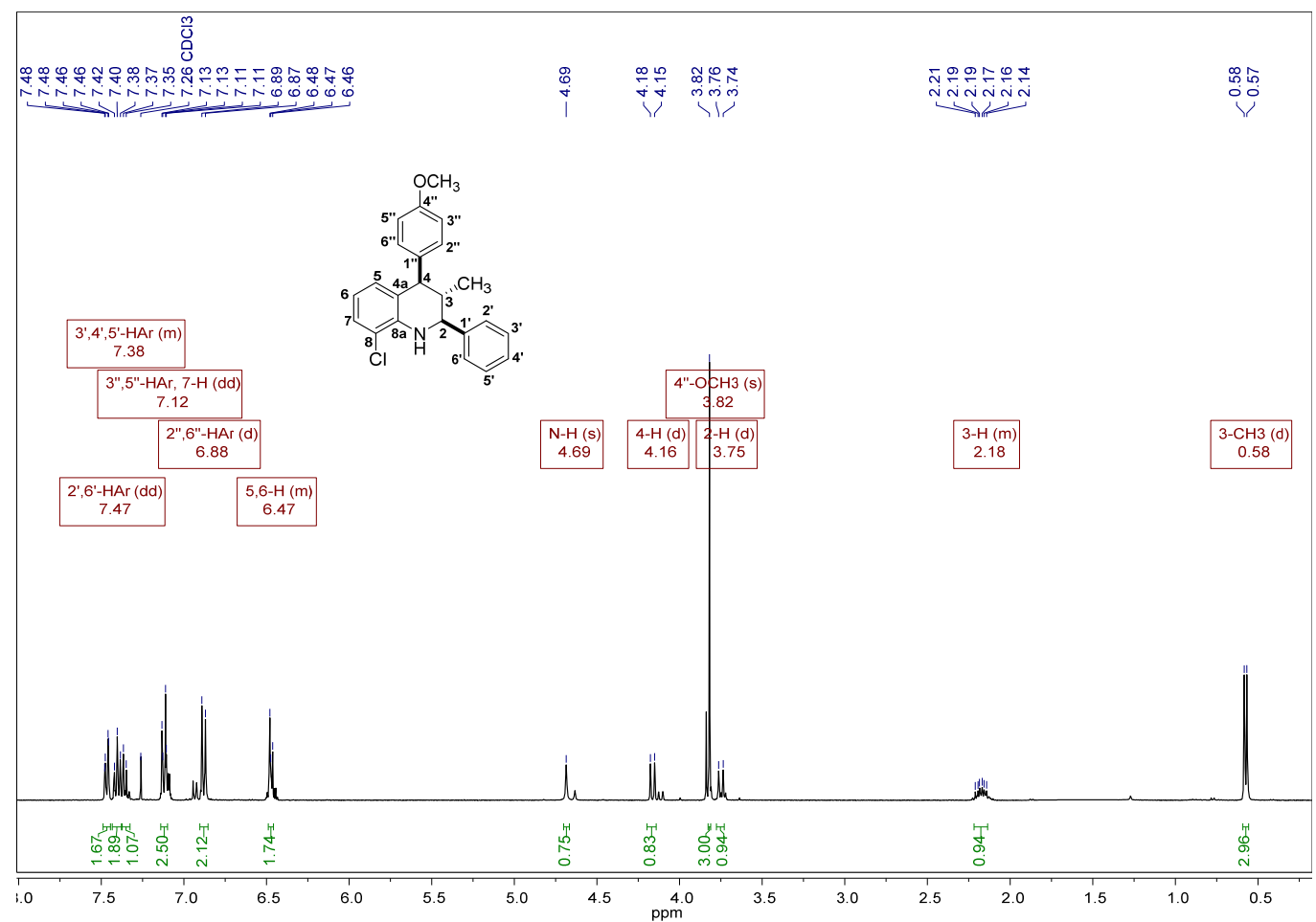

Figure S31. ${ }^{1}$ H-NMR spectra for cis-8-chloro-4-(4-methoxyphenyl)-3-methyl-2-phenyl1,2,3,4-tetrahydroquinoline (4k)

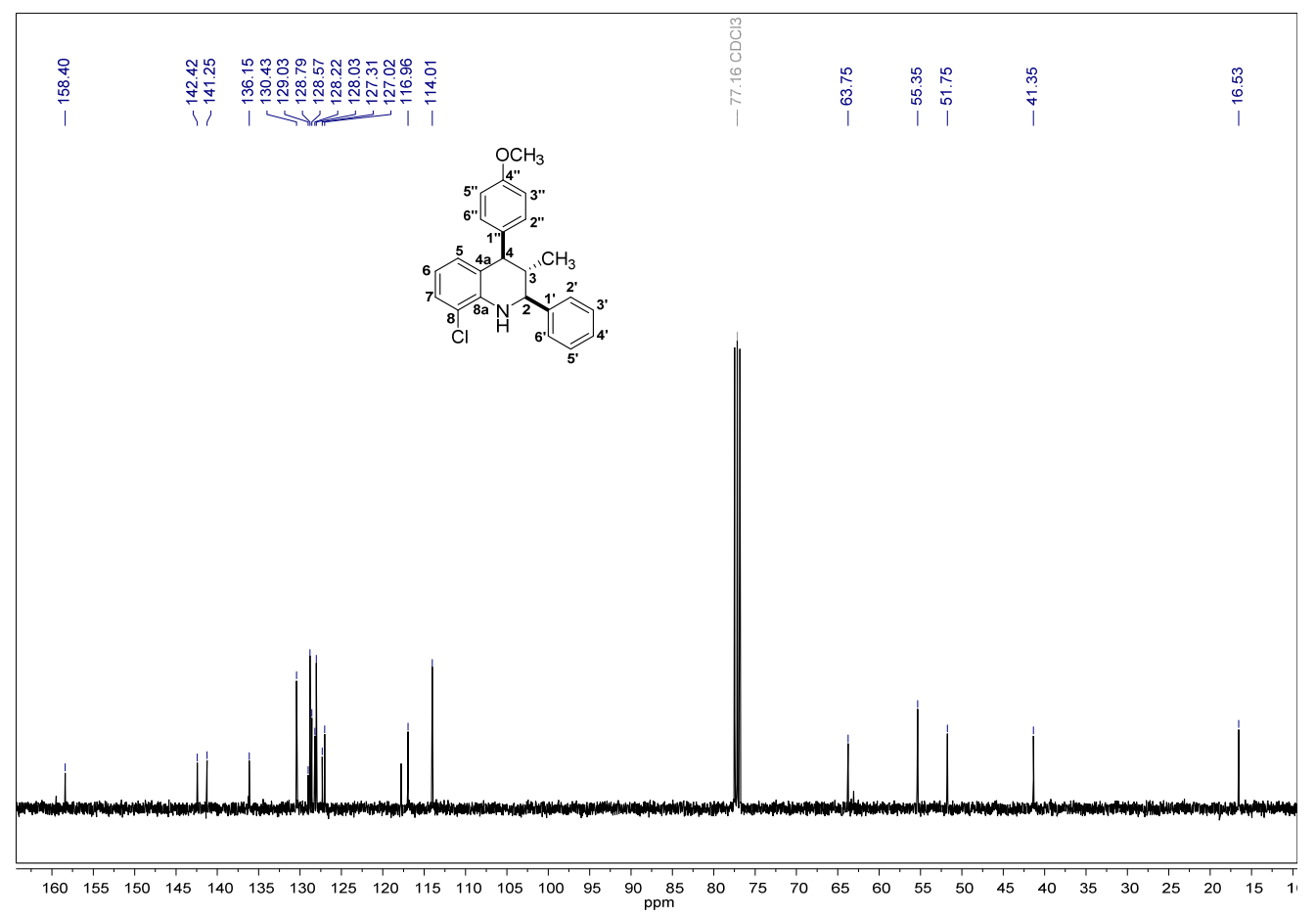

Figure S32. ${ }^{13}$ C-NMR spectra for cis-8-chloro-4-(4-methoxyphenyl)-3-methyl-2-phenyl1,2,3,4-tetrahydroquinoline (4k) 


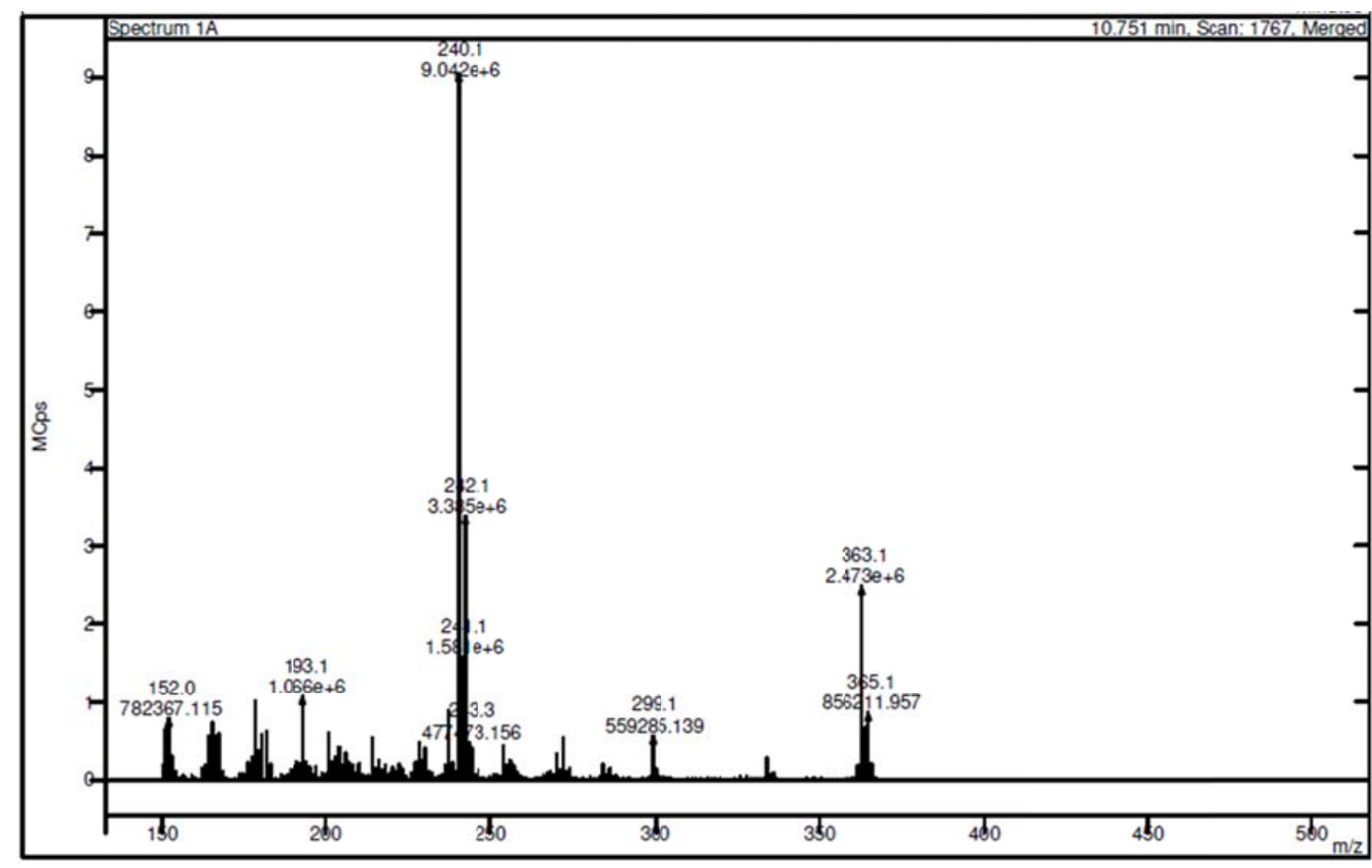

Figure S33. Mass spectra for cis-8-chloro-4-(4-methoxyphenyl)-3-methyl-2-phenyl1,2,3,4-tetrahydroquinoline (4k)

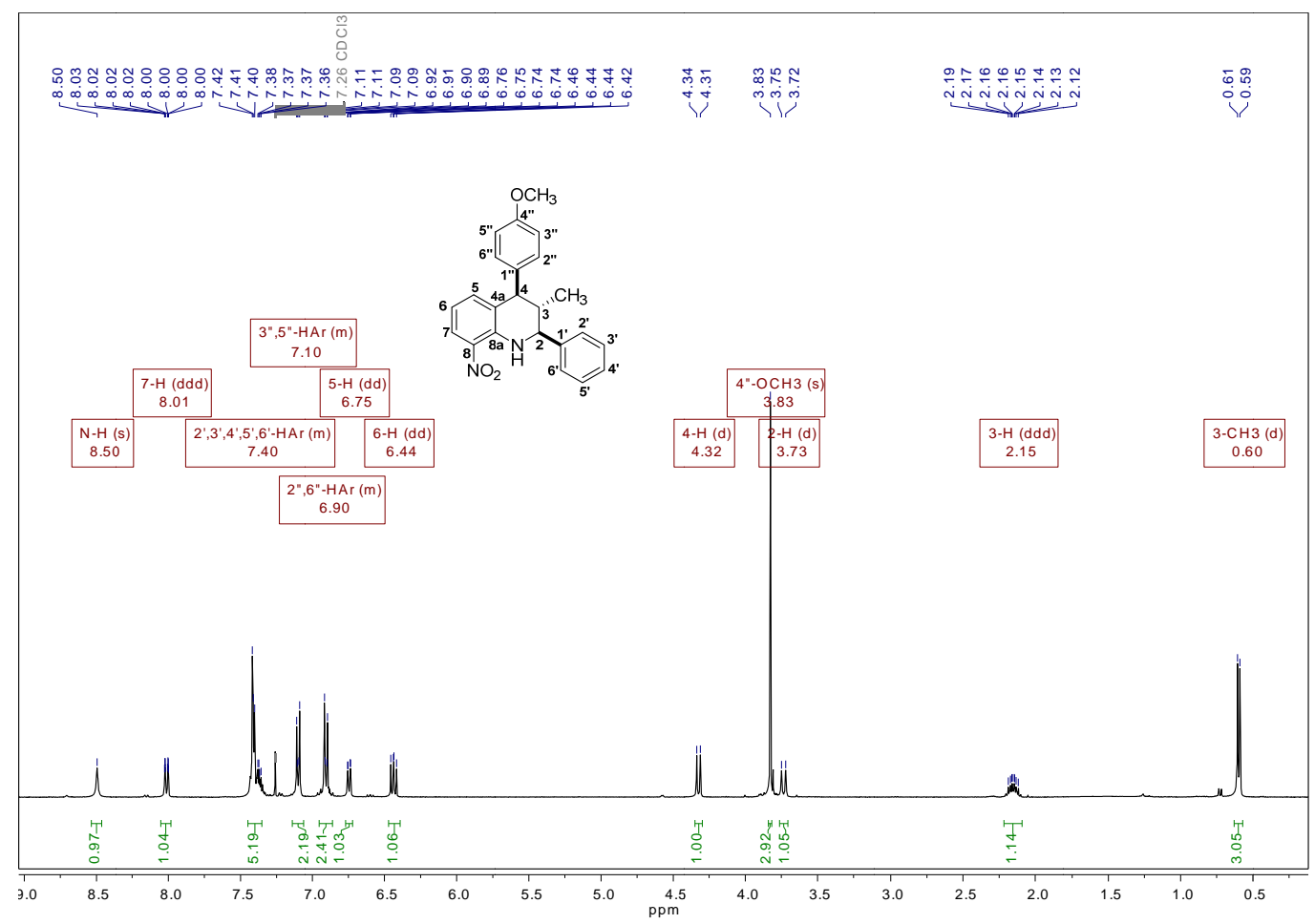

Figure S34. ${ }^{1}$ H-NMR spectra for cis-4-(4-methoxyphenyl)-3-methyl-8-nitro-2-phenyl1,2,3,4-tetrahydroquinoline (4l) 


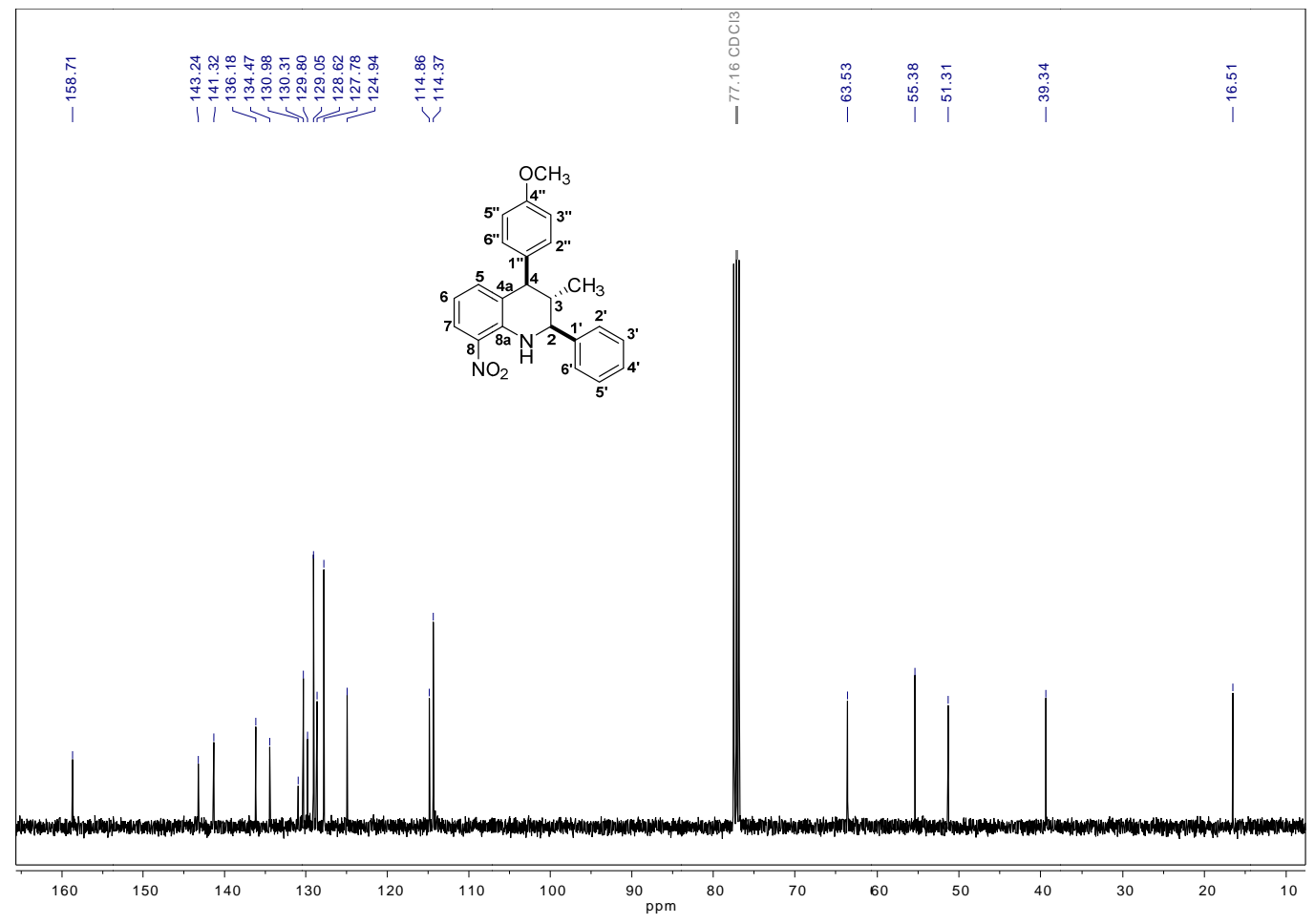

Figure S35. ${ }^{13} \mathrm{C}-\mathrm{NMR}$ spectra for cis-4-(4-methoxyphenyl)-3-methyl-8-nitro-2-phenyl1,2,3,4-tetrahydroquinoline (4l)

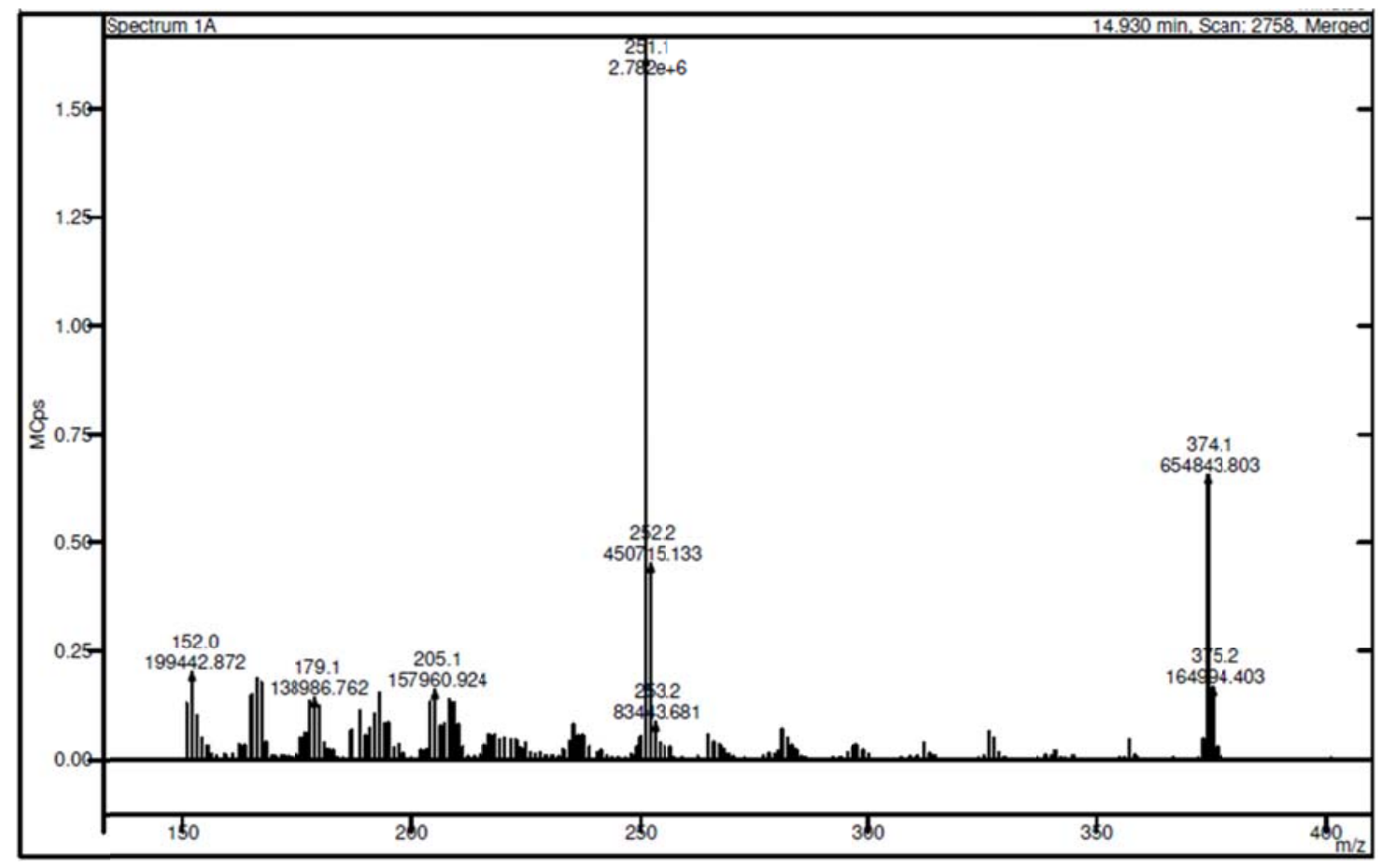

Figure S36. Mass spectra for cis-4-(4-methoxyphenyl)-3-methyl-8-nitro-2-phenyl-1,2,3,4tetrahydroquinoline (4l) 


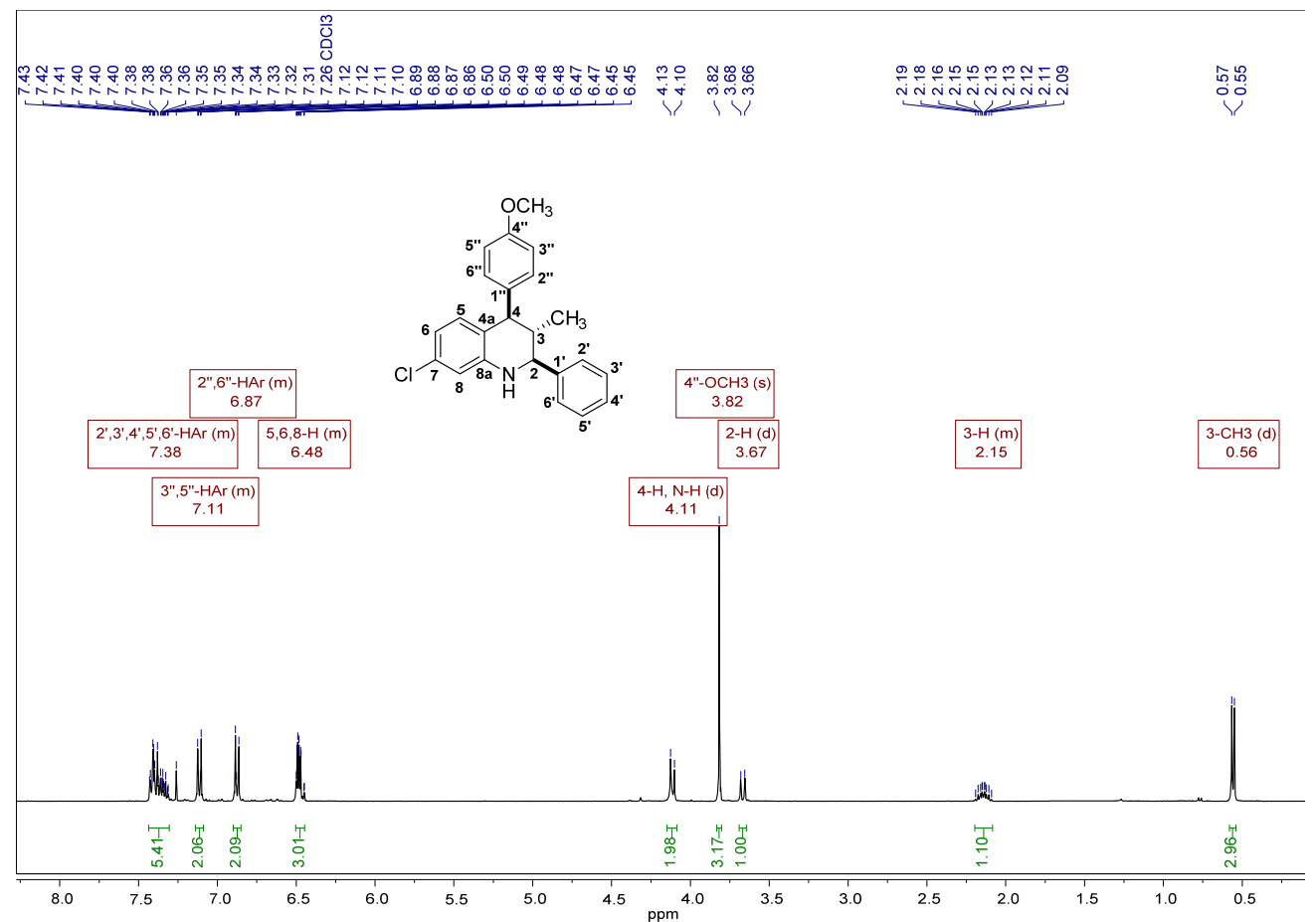

Figure S37. ${ }^{1}$ H-NMR spectra for cis-7-chloro-4-(4-methoxyphenyl)-3-methyl-2-phenyl1,2,3,4-tetrahydroquinoline (4m)

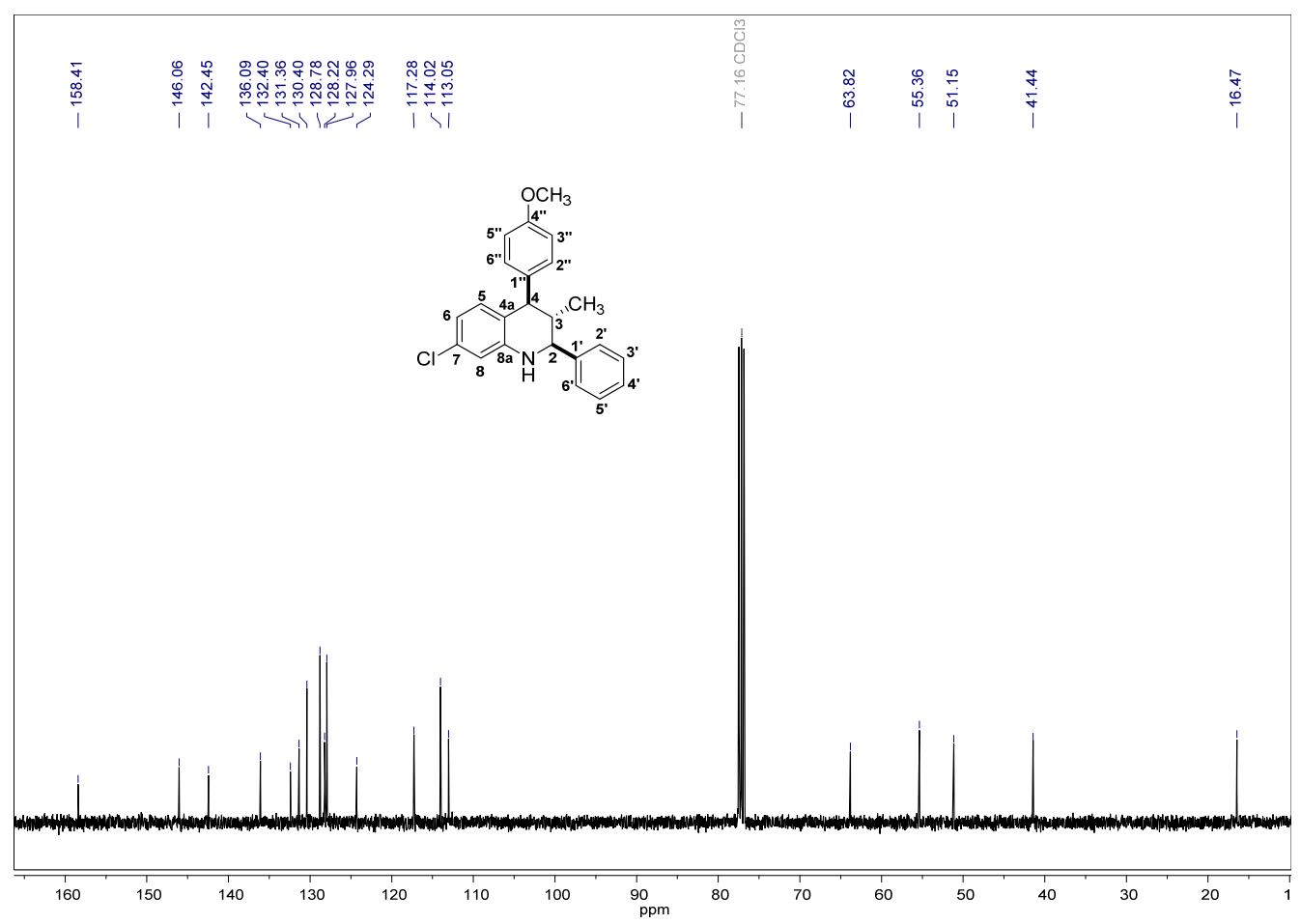

Figure S38 ${ }^{13} \mathrm{C}$-NMR spectra for cis-7-chloro-4-(4-methoxyphenyl)-3-methyl-2-phenyl1,2,3,4-tetrahydroquinoline $\mathbf{( 4 \mathbf { m } )}$ 


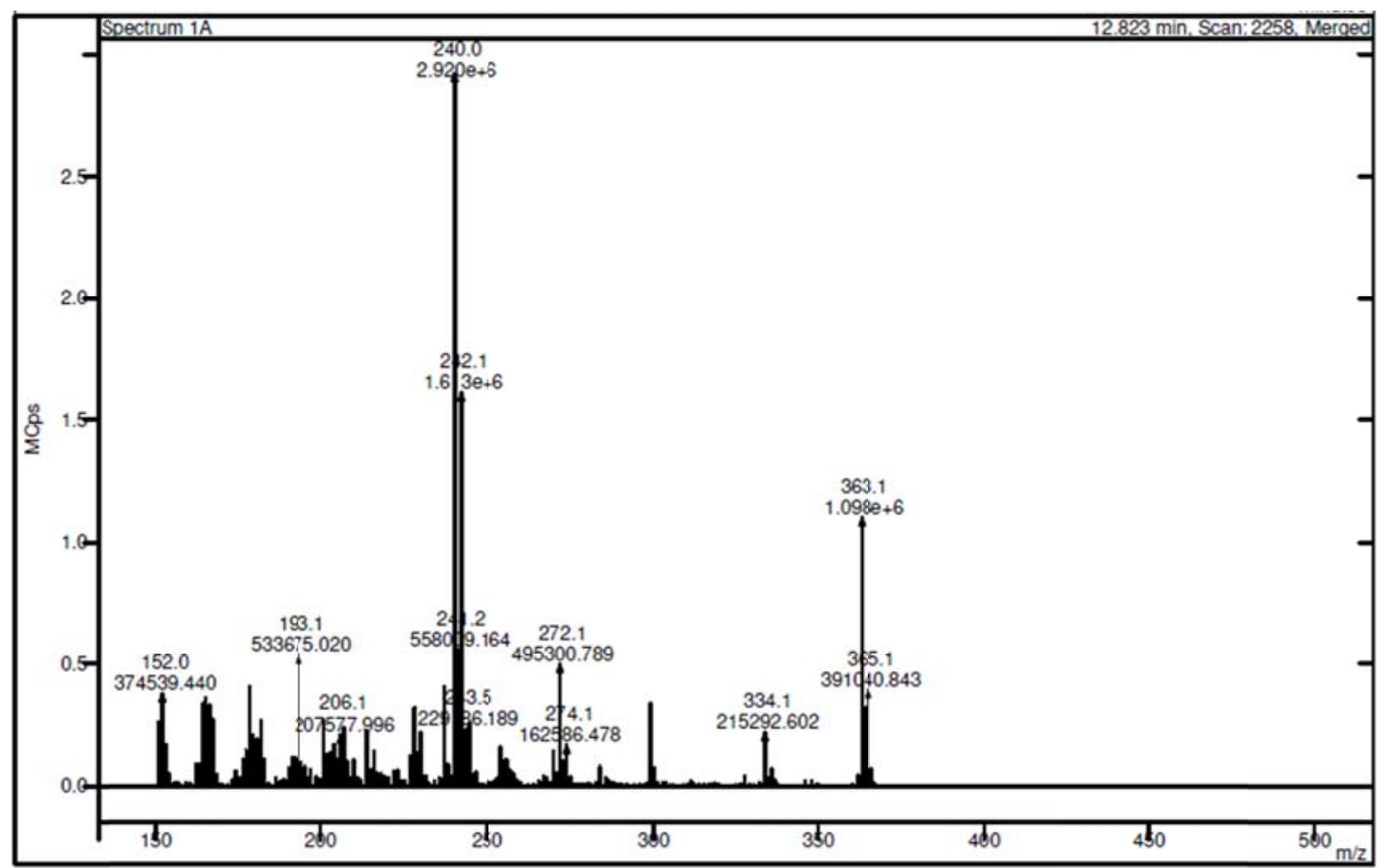

Figure S39. Mass spectra for cis-7-chloro-4-(4-methoxyphenyl)-3-methyl-2-phenyl1,2,3,4-tetrahydroquinoline (4m)

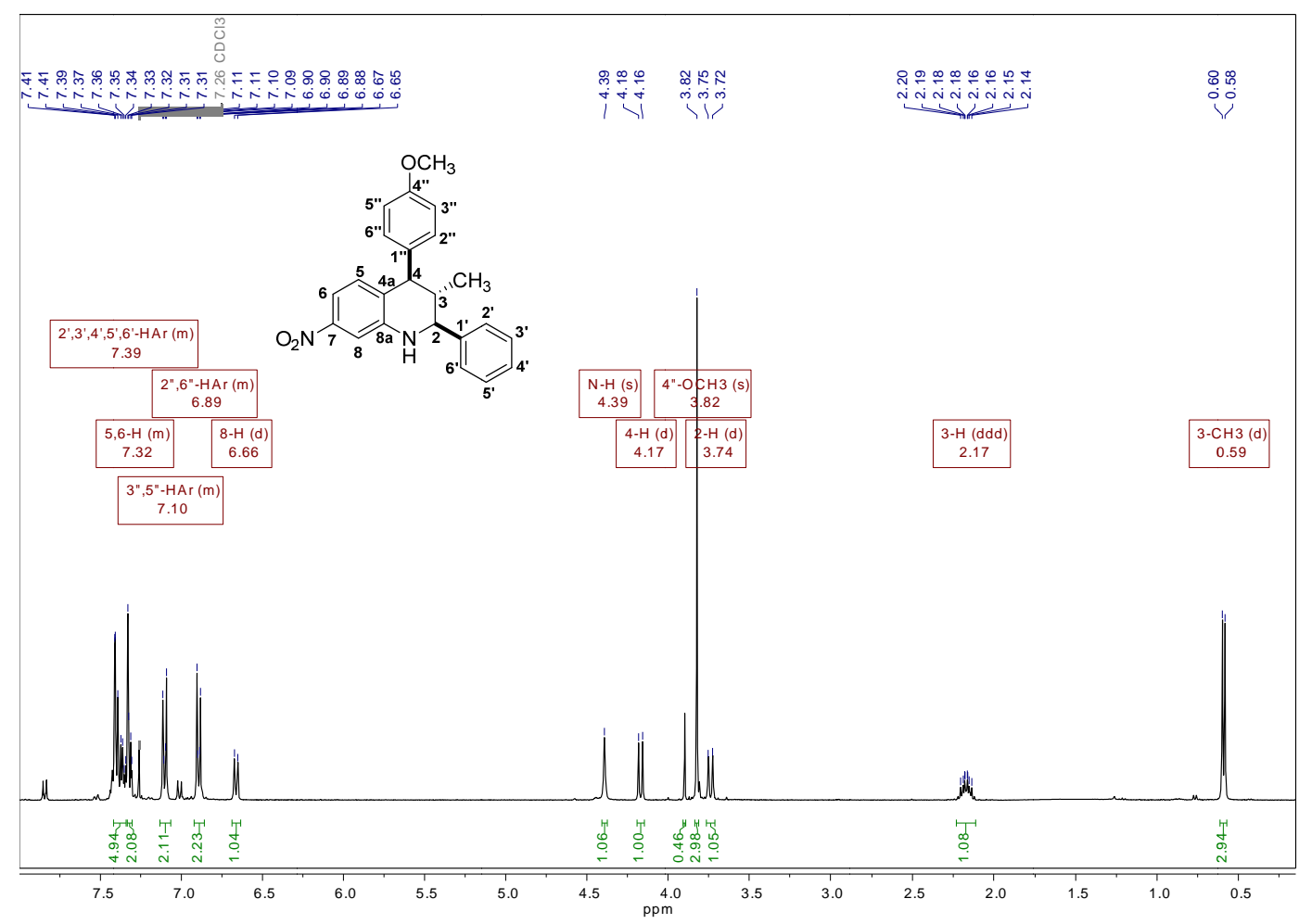

Figure S40. ${ }^{1} \mathrm{H}-\mathrm{NMR}$ spectra for cis-4-(4-methoxyphenyl)-3-methyl-7-nitro-2-phenyl1,2,3,4-tetrahydroquinoline (4n) 


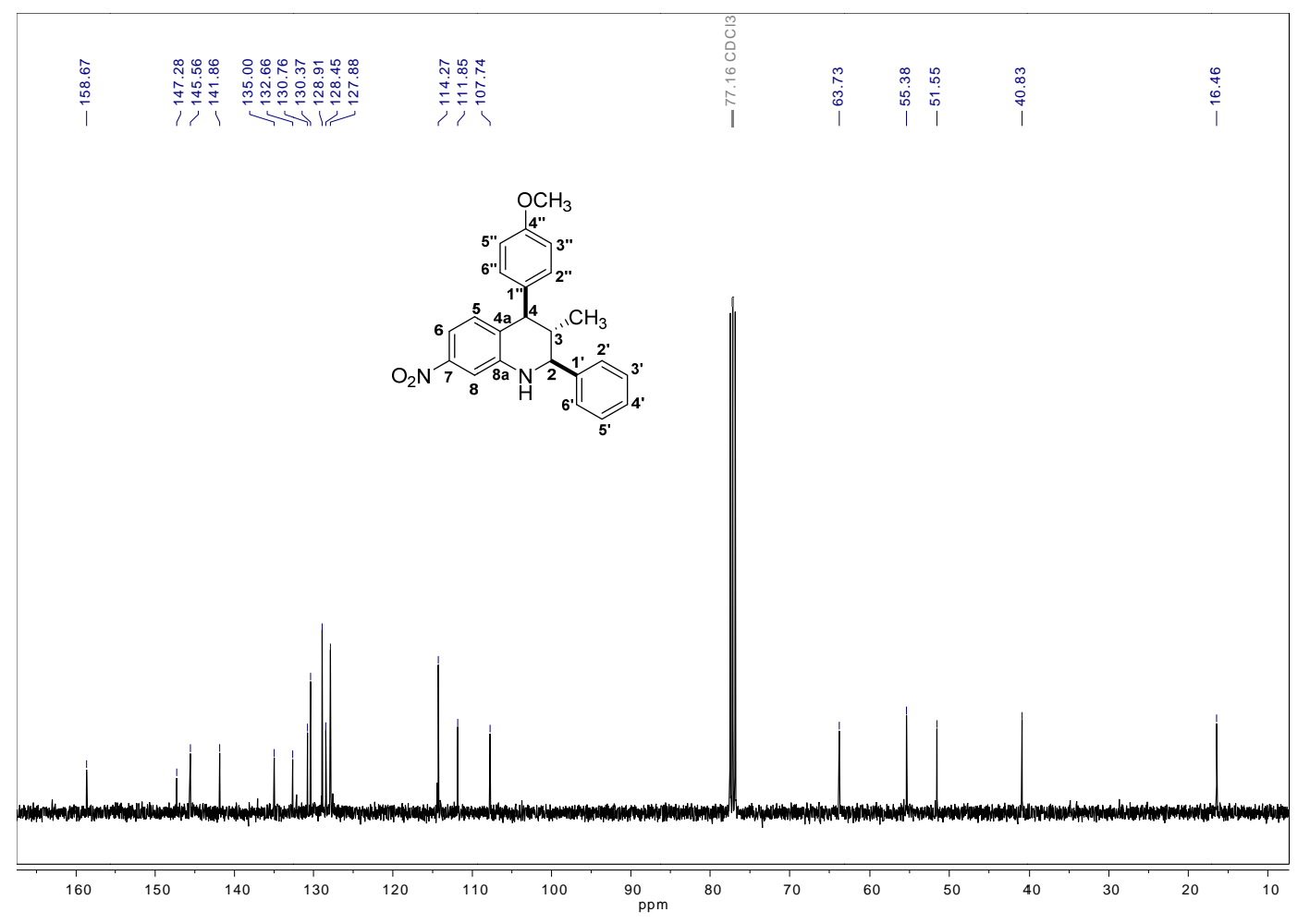

Figure S41 ${ }^{13} \mathrm{C}-\mathrm{NMR}$ spectra for cis-4-(4-methoxyphenyl)-3-methyl-7-nitro-2-phenyl1,2,3,4-tetrahydroquinoline (4n)

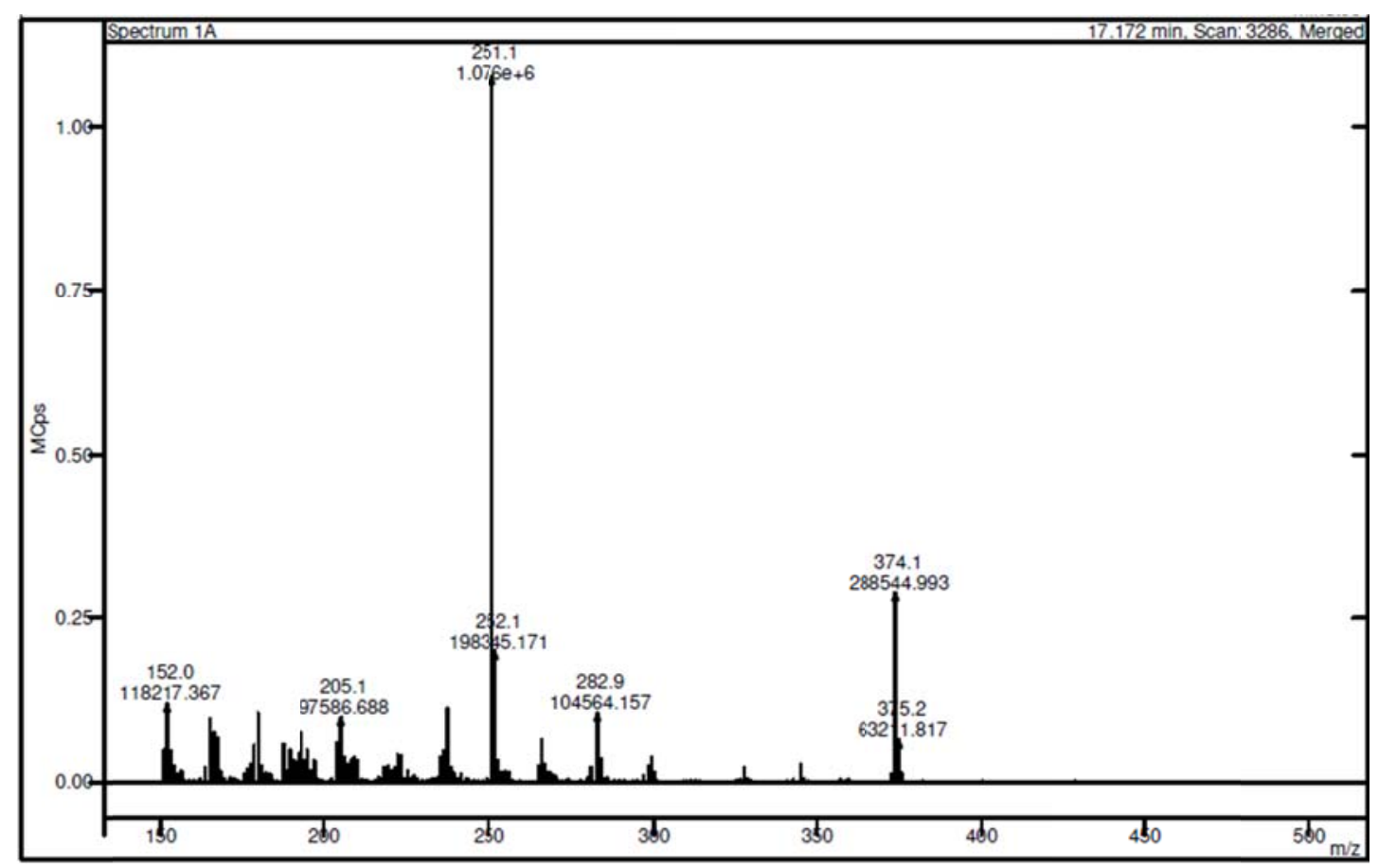

Figure S42. Mass spectra for cis-4-(4-methoxyphenyl)-3-methyl-7-nitro-2-phenyl-1,2,3,4tetrahydroquinoline (4n) 


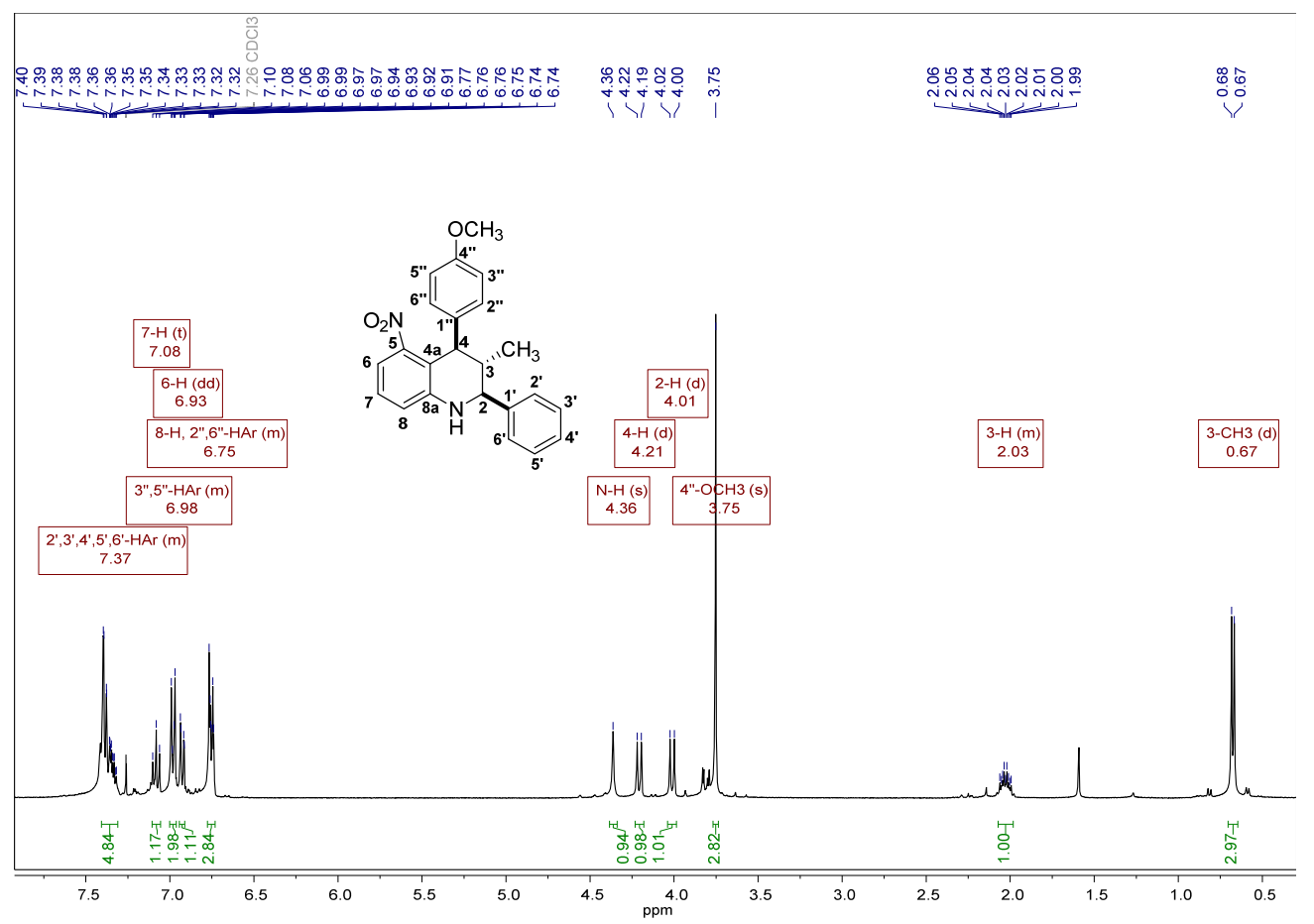

Figure S43. ${ }^{1}$ H-NMR spectra for cis-4-(4-methoxyphenyl)-3-methyl-5-nitro-2-phenyl1,2,3,4-tetrahydroquinoline (4o)

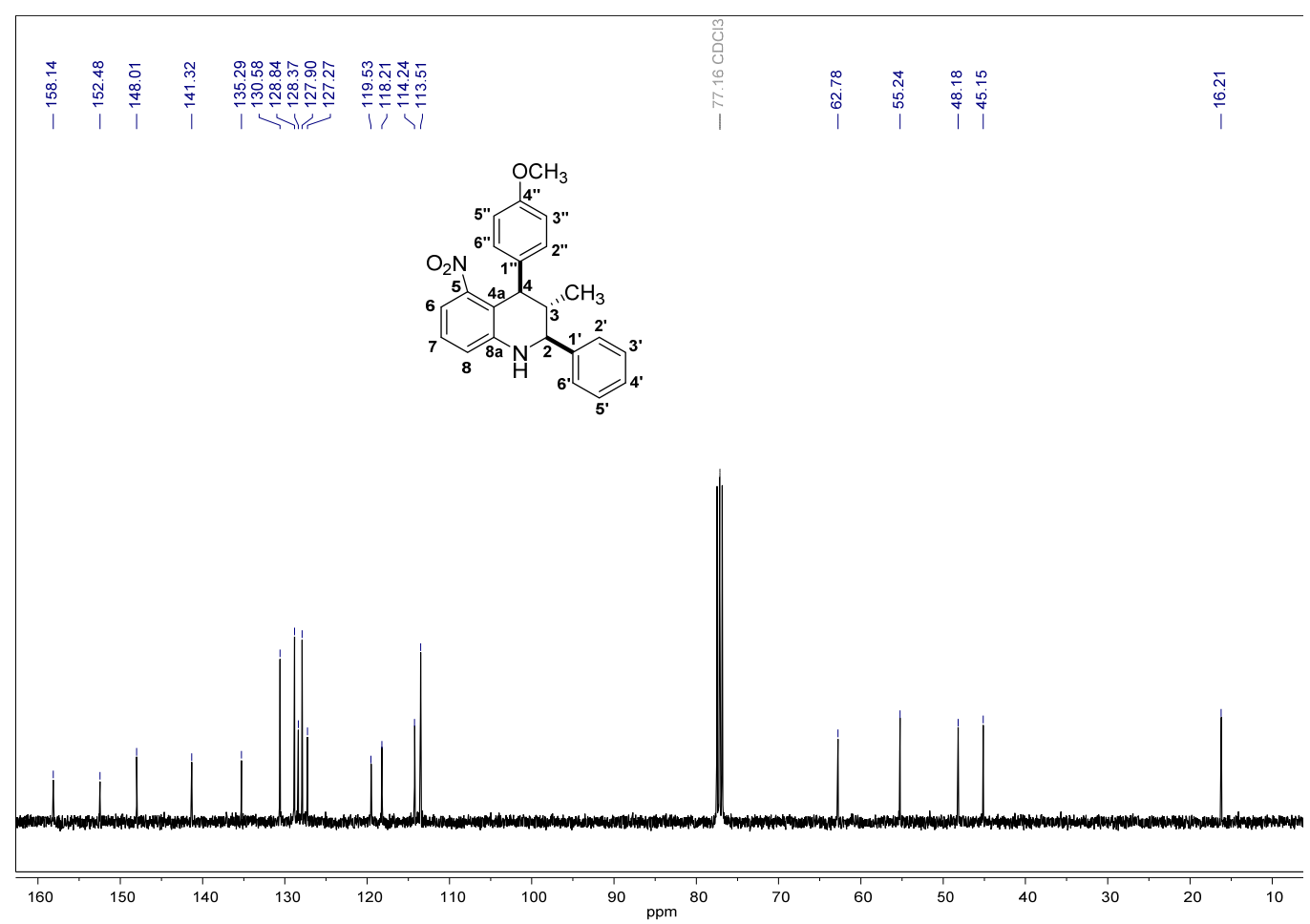

Figure S44. ${ }^{13} \mathrm{C}$-NMR spectra for cis-4-(4-methoxyphenyl)-3-methyl-5-nitro-2-phenyl1,2,3,4-tetrahydroquinoline (4o) 


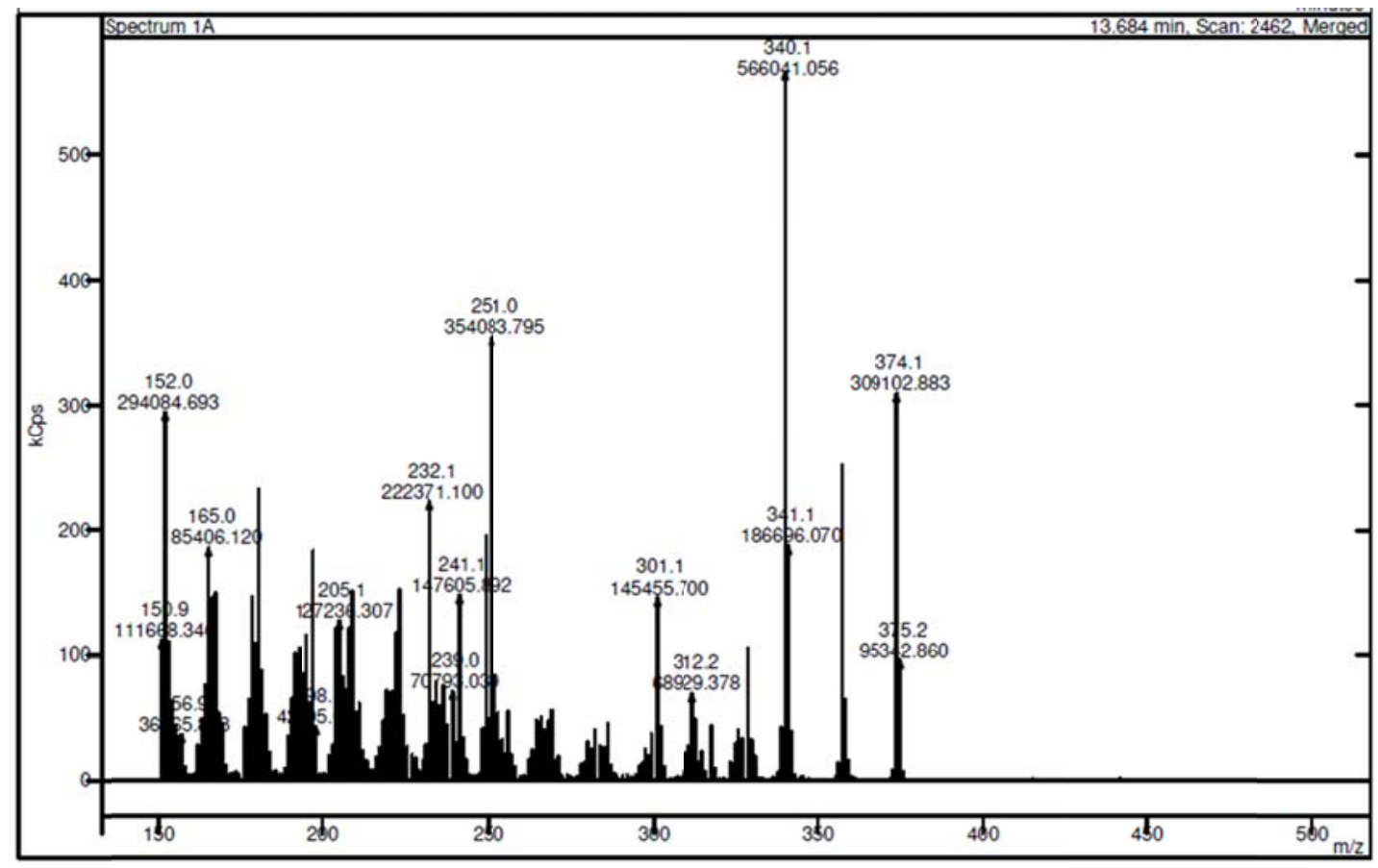

Figure S45. Mass spectra for cis-4-(4-methoxyphenyl)-3-methyl-5-nitro-2-phenyl-1,2,3,4tetrahydroquinoline (4o)

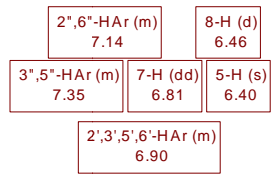

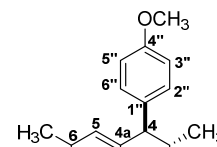
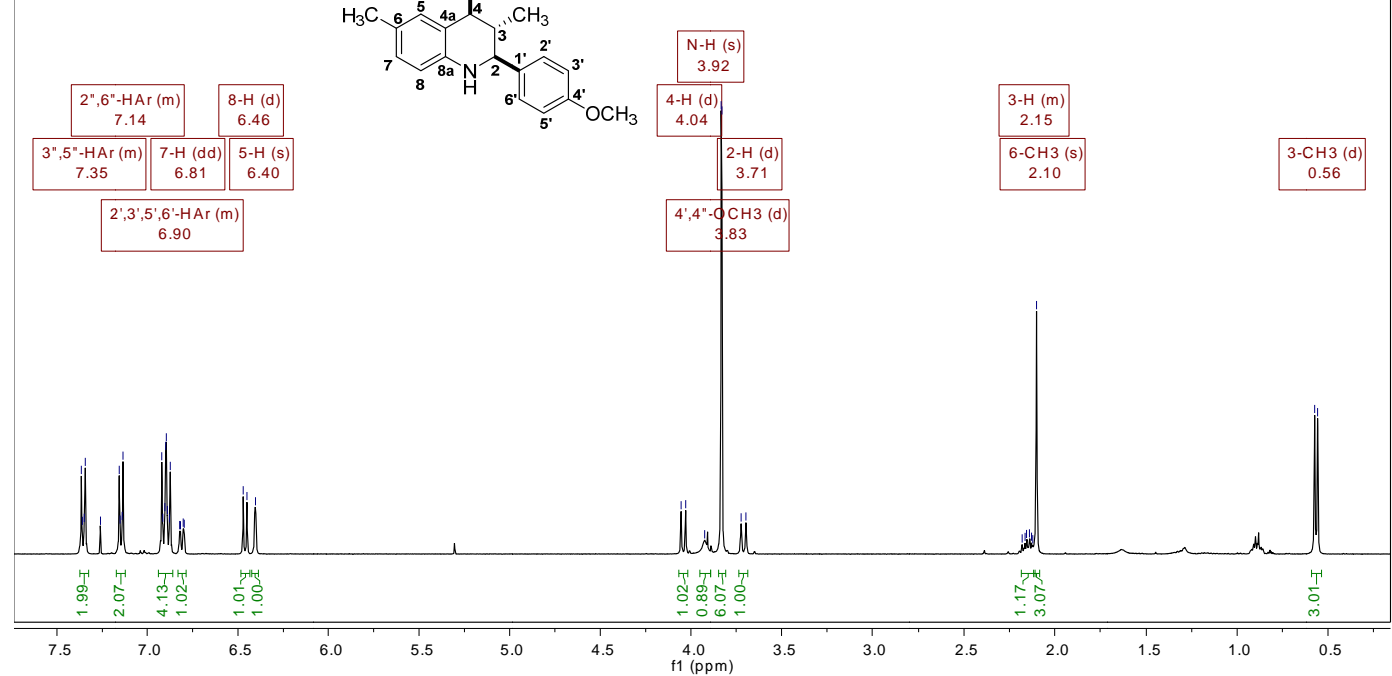

Figure S46. ${ }^{1}$ H-NMR spectra for cis-2,4-bis(4-methoxyphenyl)-3,6-dimethyl-1,2,3,4tetrahydroquinoline (4p) 


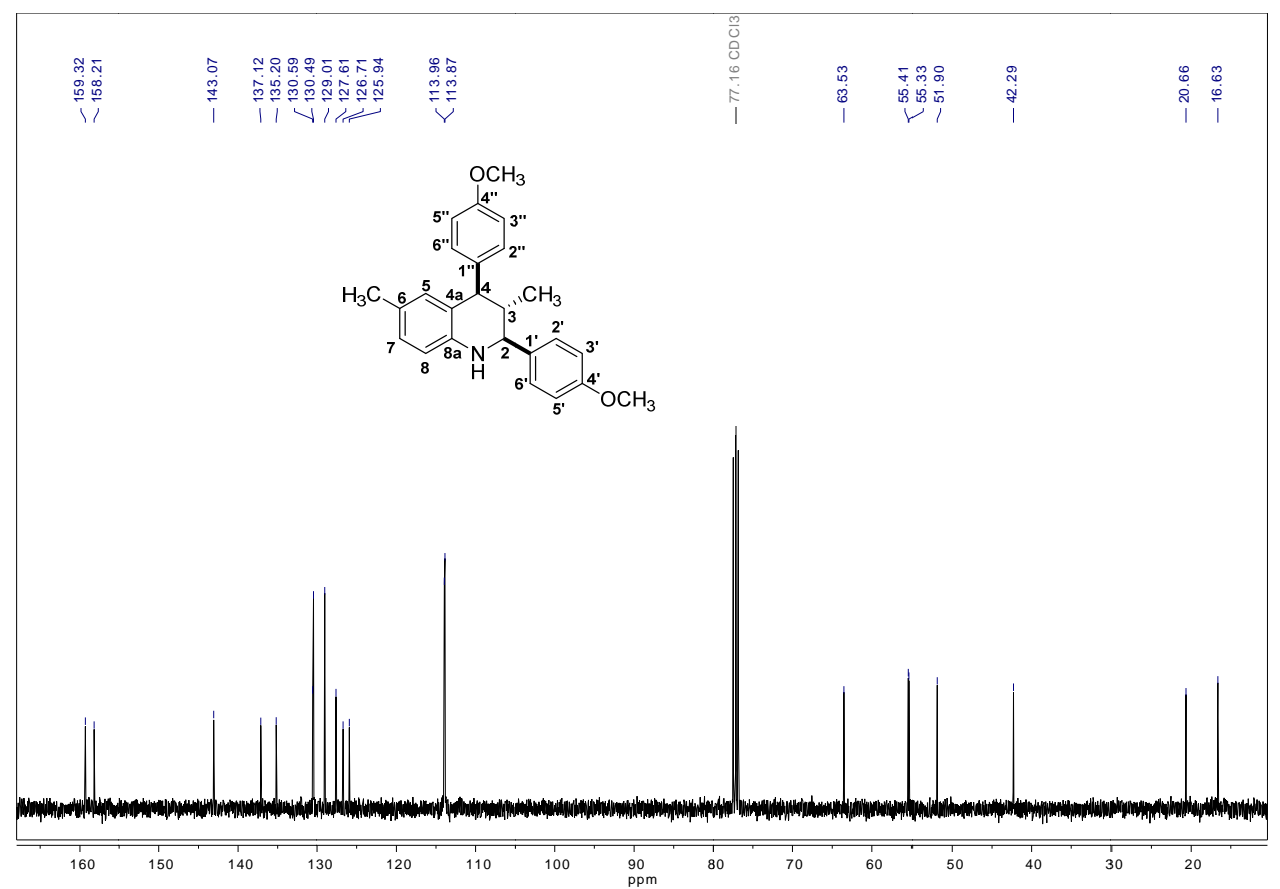

Figure S47. ${ }^{13}$ C-NMR spectra for cis-2,4-bis(4-methoxyphenyl)-3,6-dimethyl-1,2,3,4tetrahydroquinoline (4p)

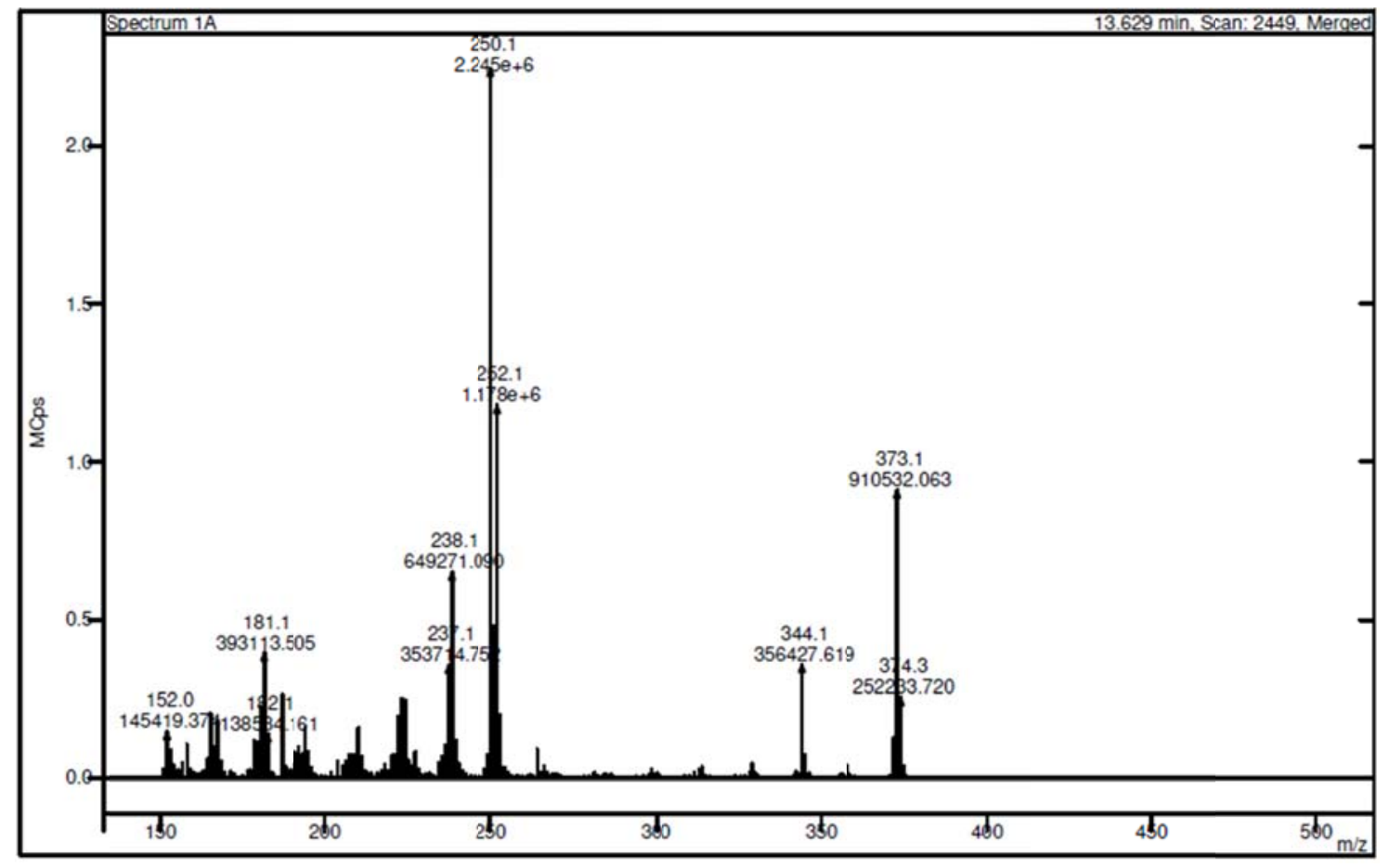

Figure S48. Mass spectra for cis-2,4-bis(4-methoxyphenyl)-3,6-dimethyl-1,2,3,4tetrahydroquinoline (4p) 


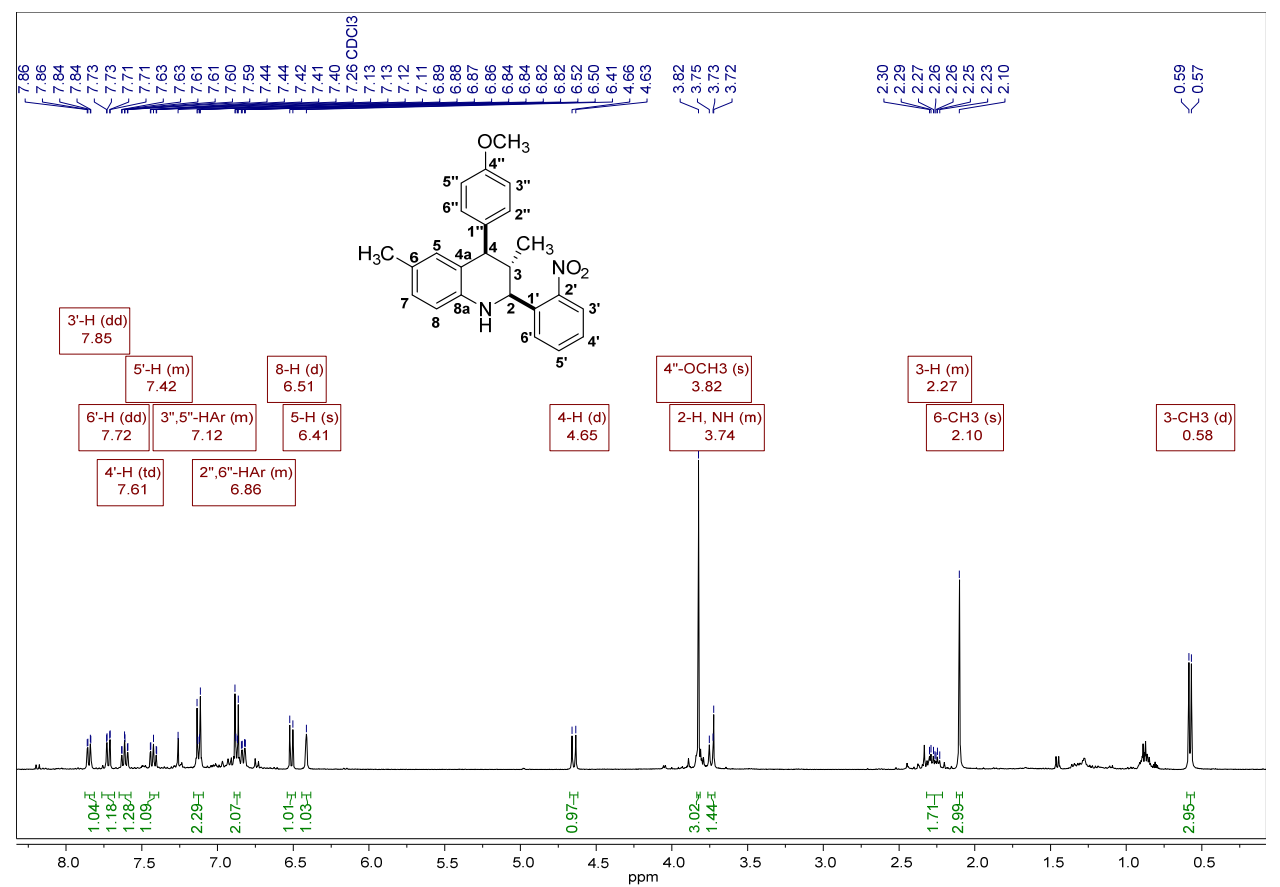

Figure S49. ${ }^{1}$ H-NMR spectra for cis-4-(4-methoxyphenyl)-3,6-dimethyl-2-(2-nitrophenyl)1,2,3,4-tetrahydroquinoline (4q)

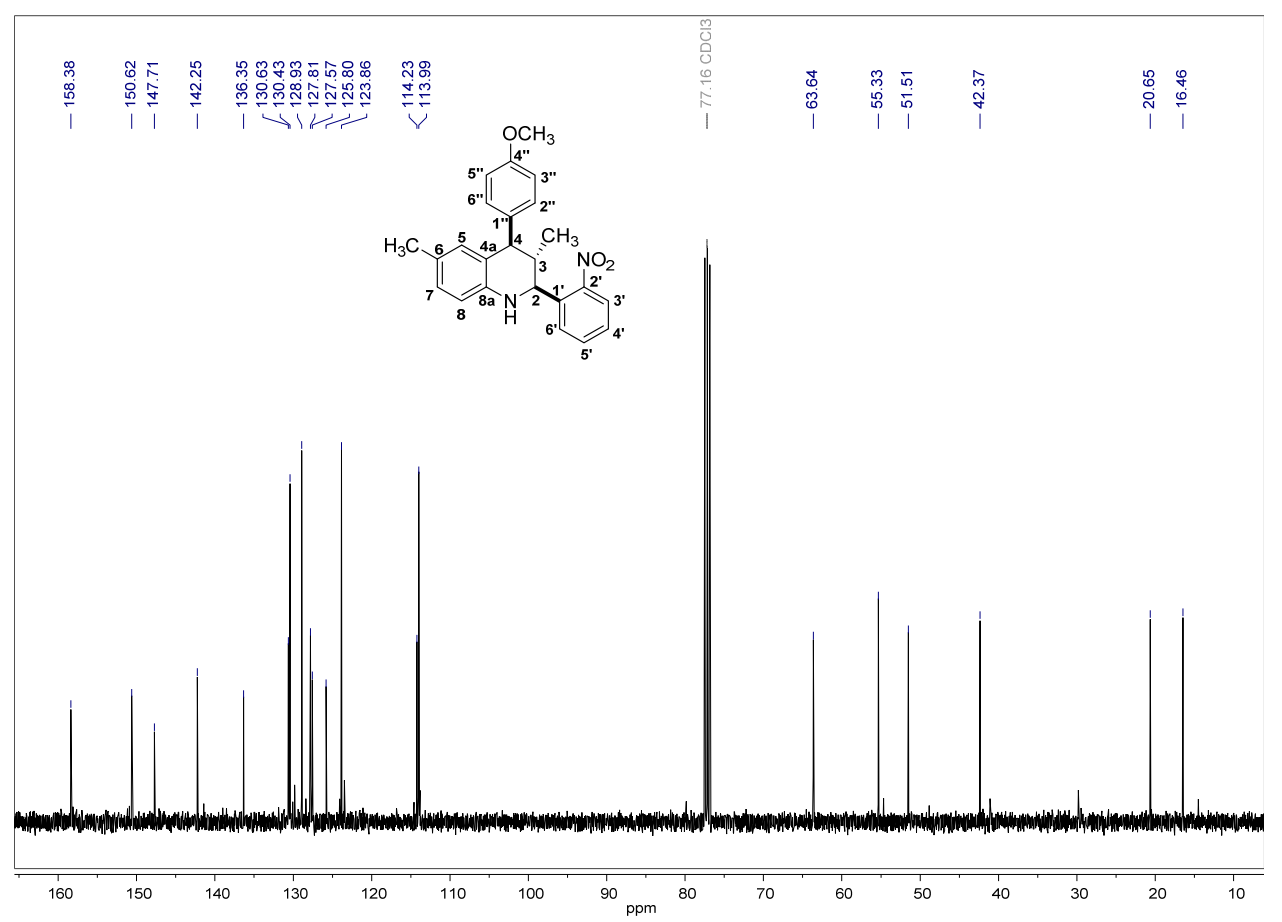

Figure S50. ${ }^{13} \mathrm{C}-\mathrm{NMR}$ spectra for cis-4-(4-methoxyphenyl)-3,6-dimethyl-2-(2nitrophenyl)-1,2,3,4-tetrahydroquinoline (4q) 


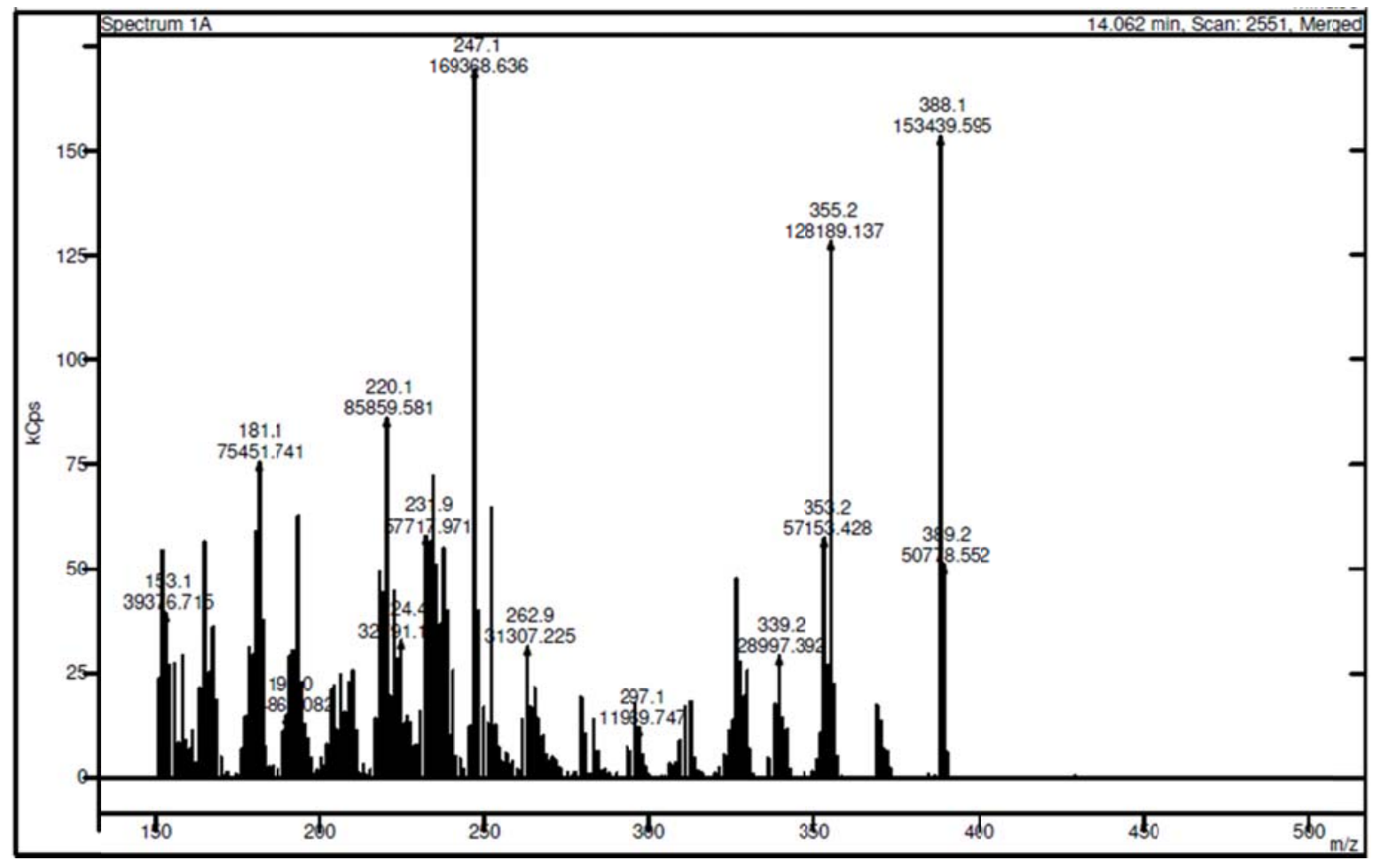

Figure S51. Mass spectra for cis-4-(4-methoxyphenyl)-3,6-dimethyl-2-(2-nitrophenyl)1,2,3,4-tetrahydroquinoline (4q)

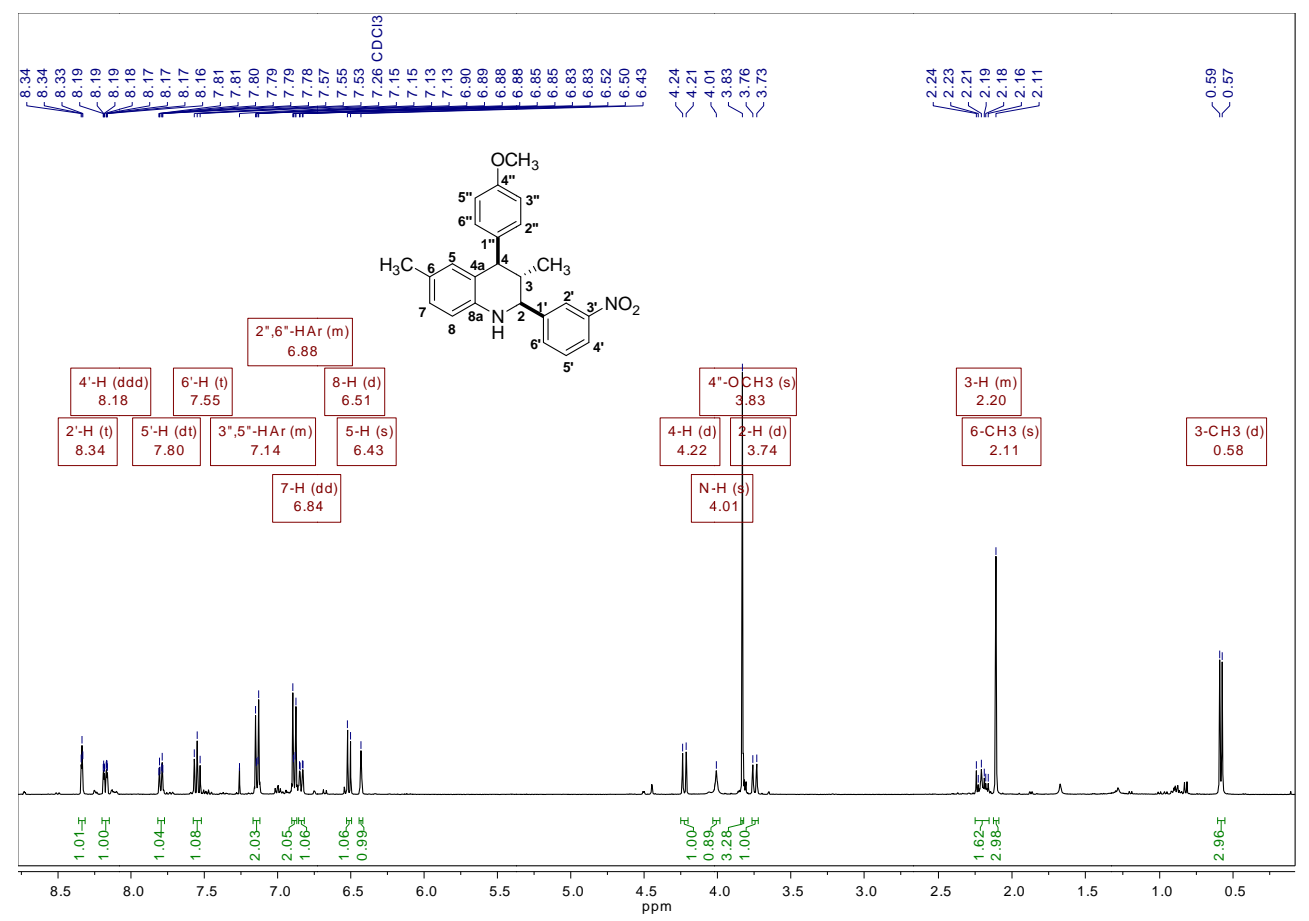

Figure S52. ${ }^{1}$ H-NMR spectra for cis-4-(4-methoxyphenyl)-3,6-dimethyl-2-(3-nitrophenyl)1,2,3,4-tetrahydroquinoline (4r) 


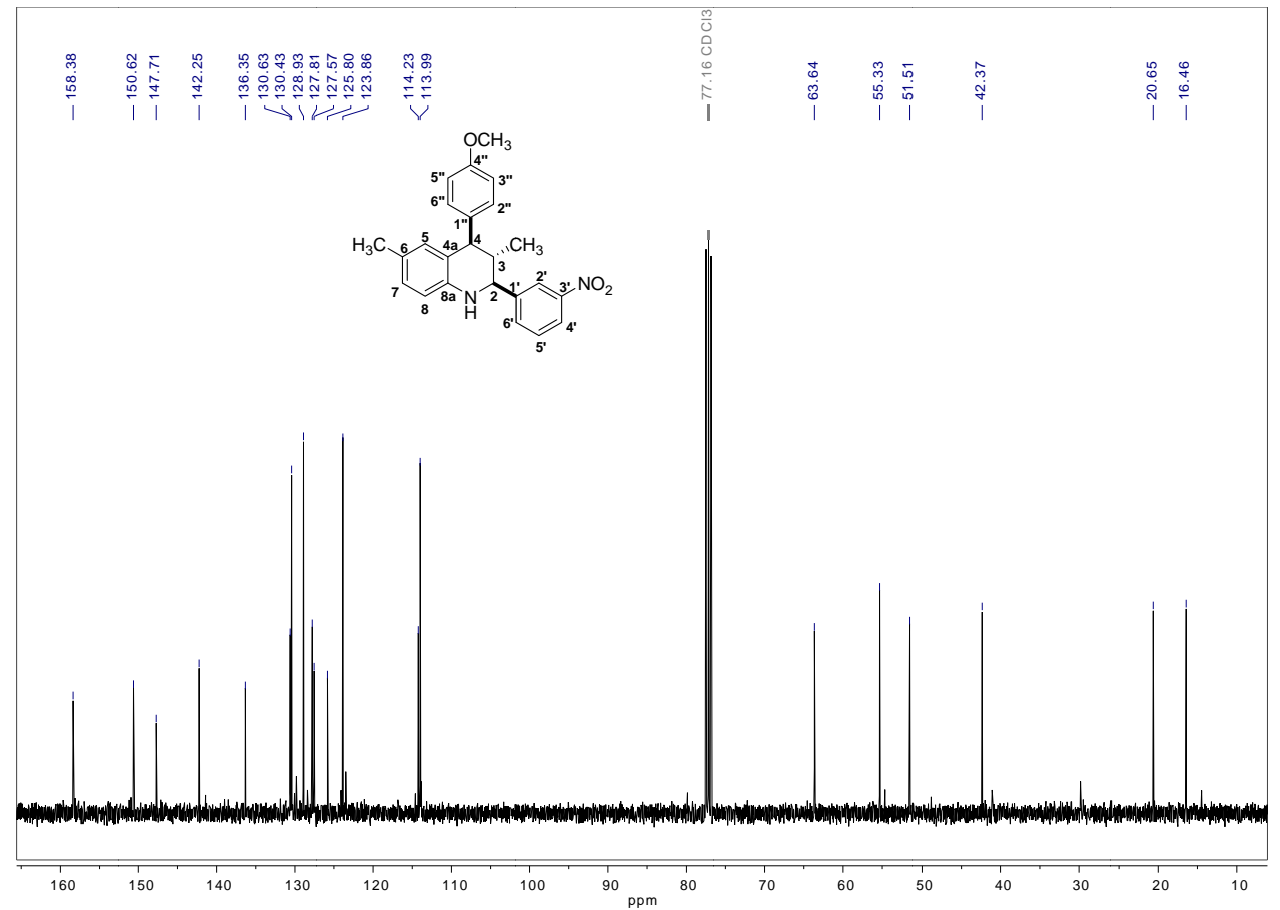

Figure S53. ${ }^{13} \mathrm{C}-\mathrm{NMR}$ spectra for cis-4-(4-methoxyphenyl)-3,6-dimethyl-2-(3nitrophenyl)-1,2,3,4-tetrahydroquinoline (4r)

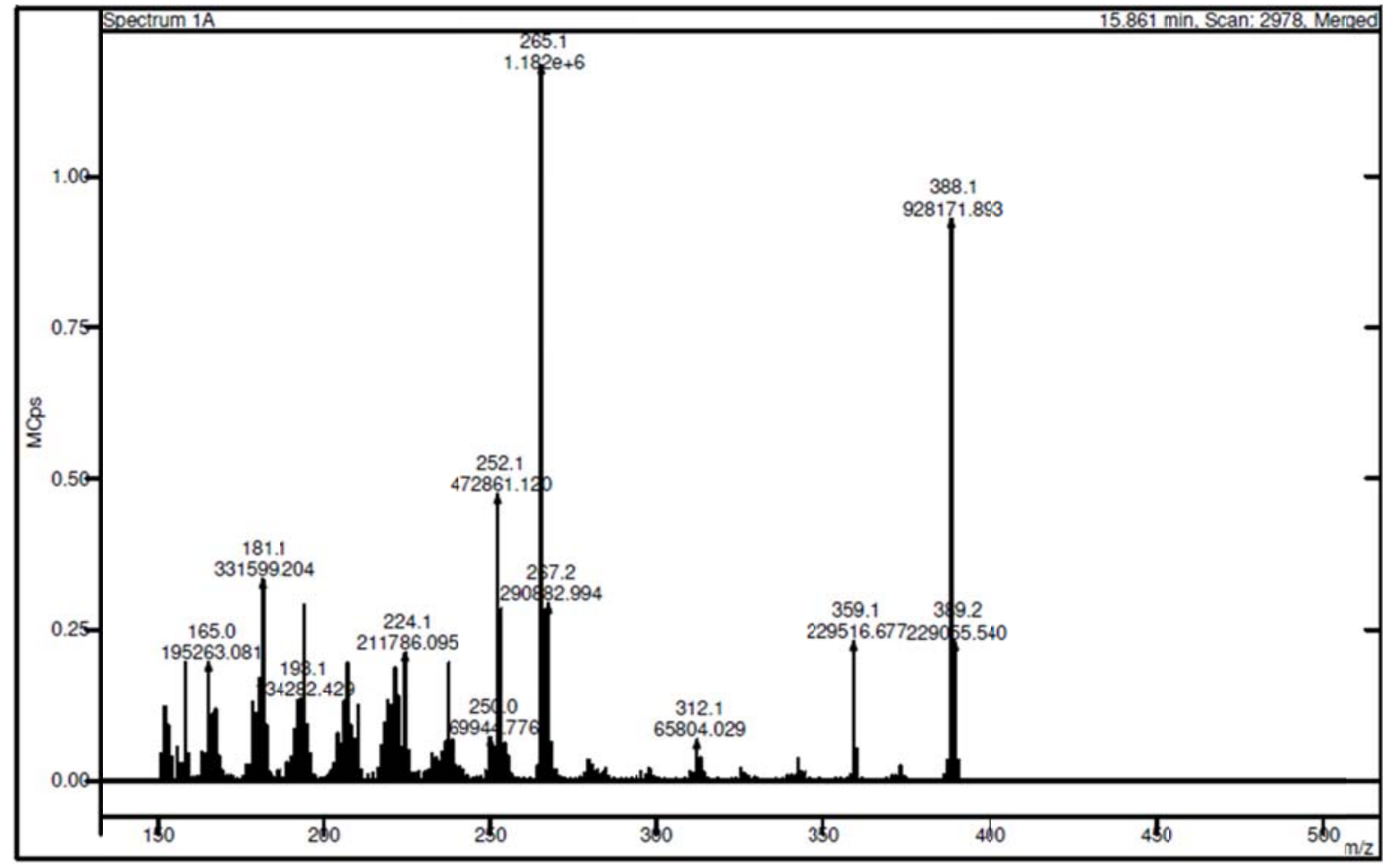

Figure S54. Mass spectra for cis-4-(4-methoxyphenyl)-3,6-dimethyl-2-(3-nitrophenyl)1,2,3,4-tetrahydroquinoline (4r) 


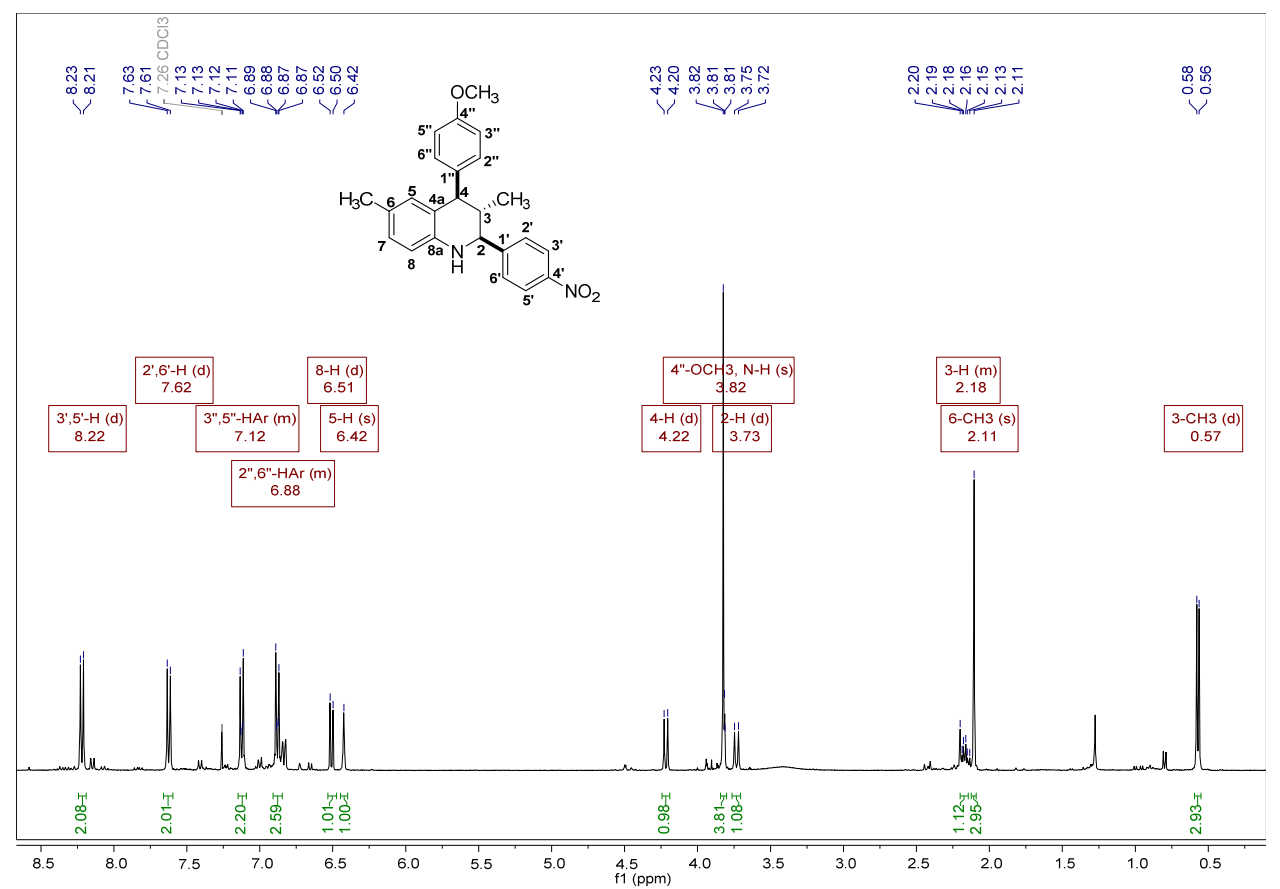

Figure S55. ${ }^{1} \mathrm{H}-\mathrm{NMR}$ spectra for cis-4-(4-methoxyphenyl)-3,6-dimethyl-2-(4-nitrophenyl)1,2,3,4-tetrahydroquinoline (4s)

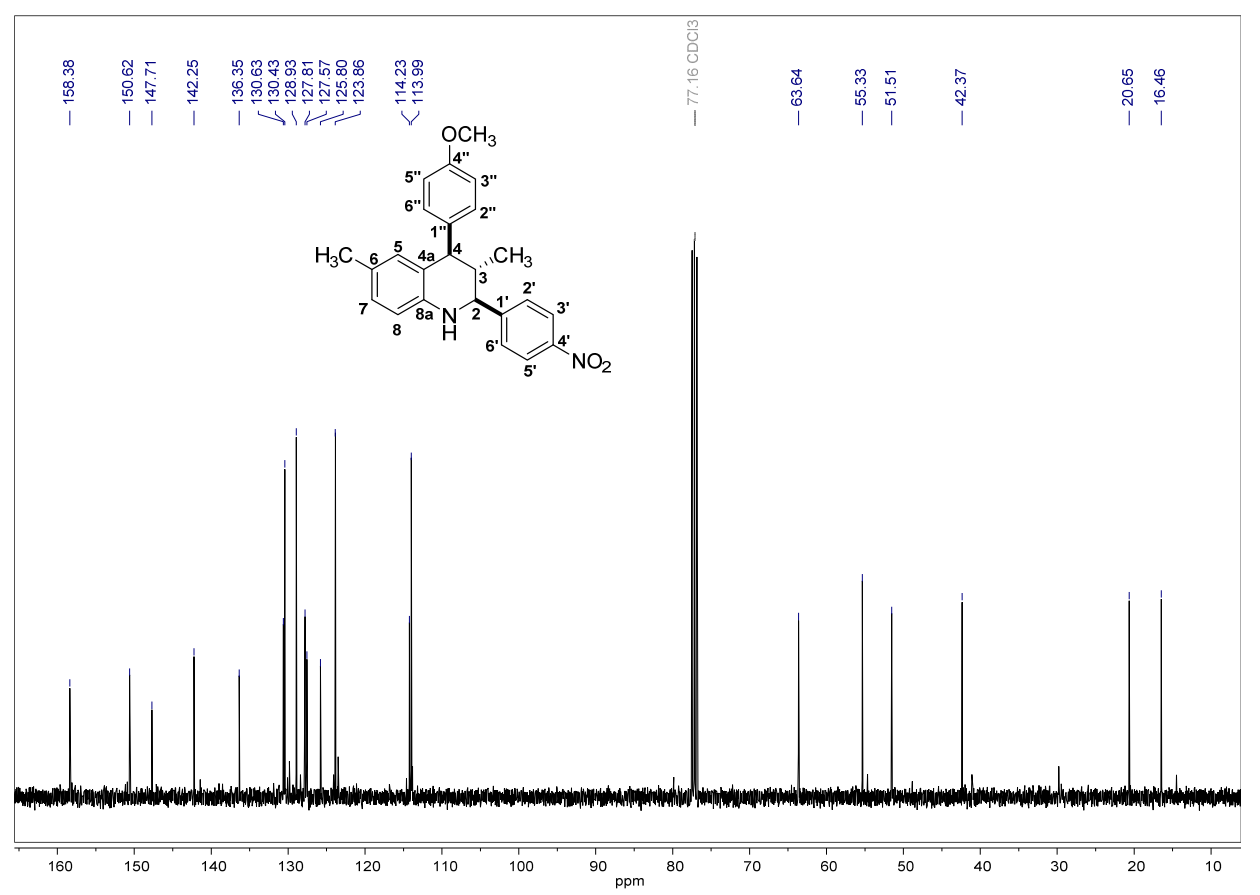

Figure S56. ${ }^{13} \mathrm{C}-\mathrm{NMR}$ spectra for cis-4-(4-methoxyphenyl)-3,6-dimethyl-2-(4nitrophenyl)-1,2,3,4-tetrahydroquinoline (4s) 


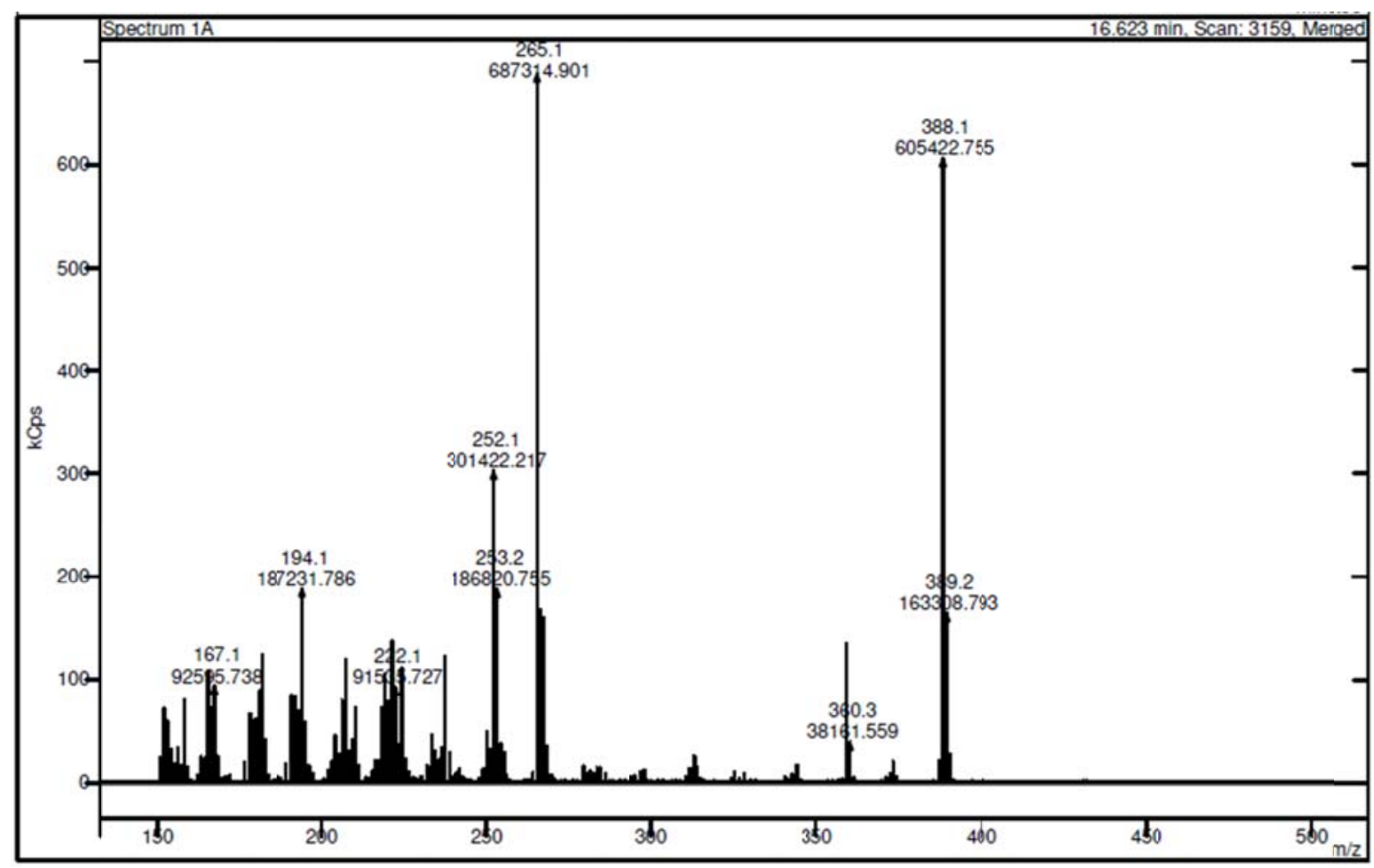

Figure S57. Mass spectra for cis-4-(4-methoxyphenyl)-3,6-dimethyl-2-(4-nitrophenyl)1,2,3,4-tetrahydroquinoline (4s)

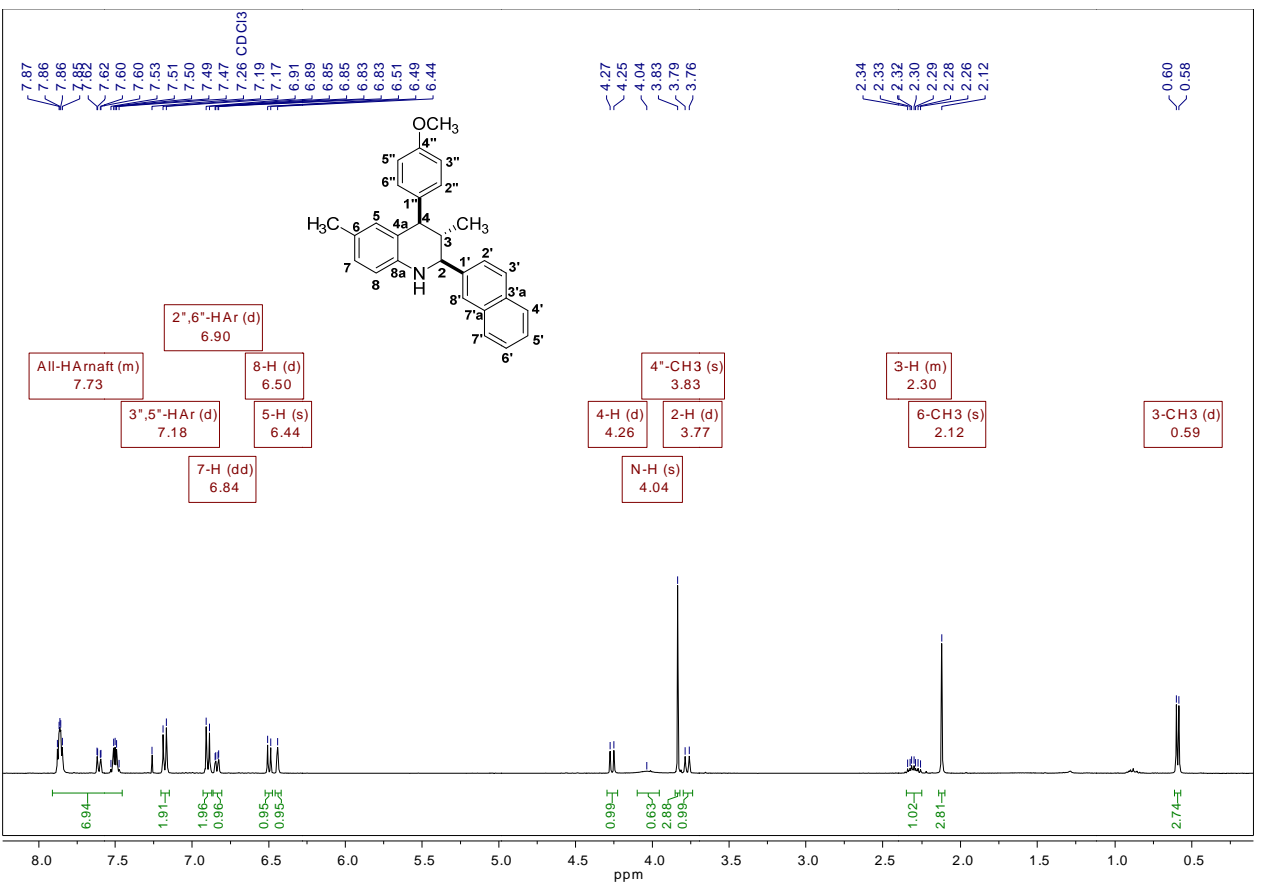

Figure S58. ${ }^{1} \mathrm{H}-\mathrm{NMR}$ spectra for cis-4-(4-methoxyphenyl)-3,6-dimethyl-2-(naphthalen-2yl)-1,2,3,4-tetrahydroquinoline (4t) 


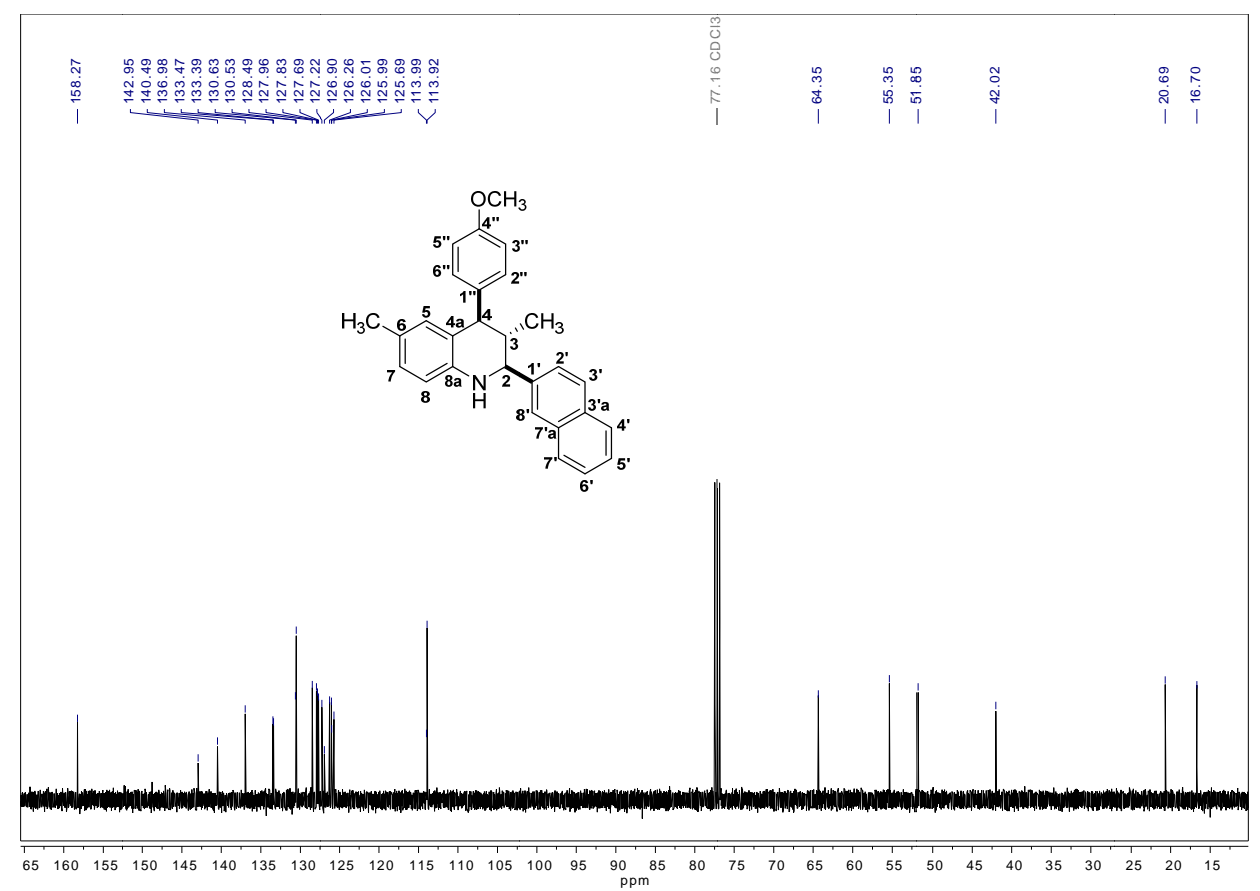

Figure S59. ${ }^{13} \mathrm{C}-\mathrm{NMR}$ spectra for cis-4-(4-methoxyphenyl)-3,6-dimethyl-2-(naphthalen-2yl)-1,2,3,4-tetrahydroquinoline (4t)

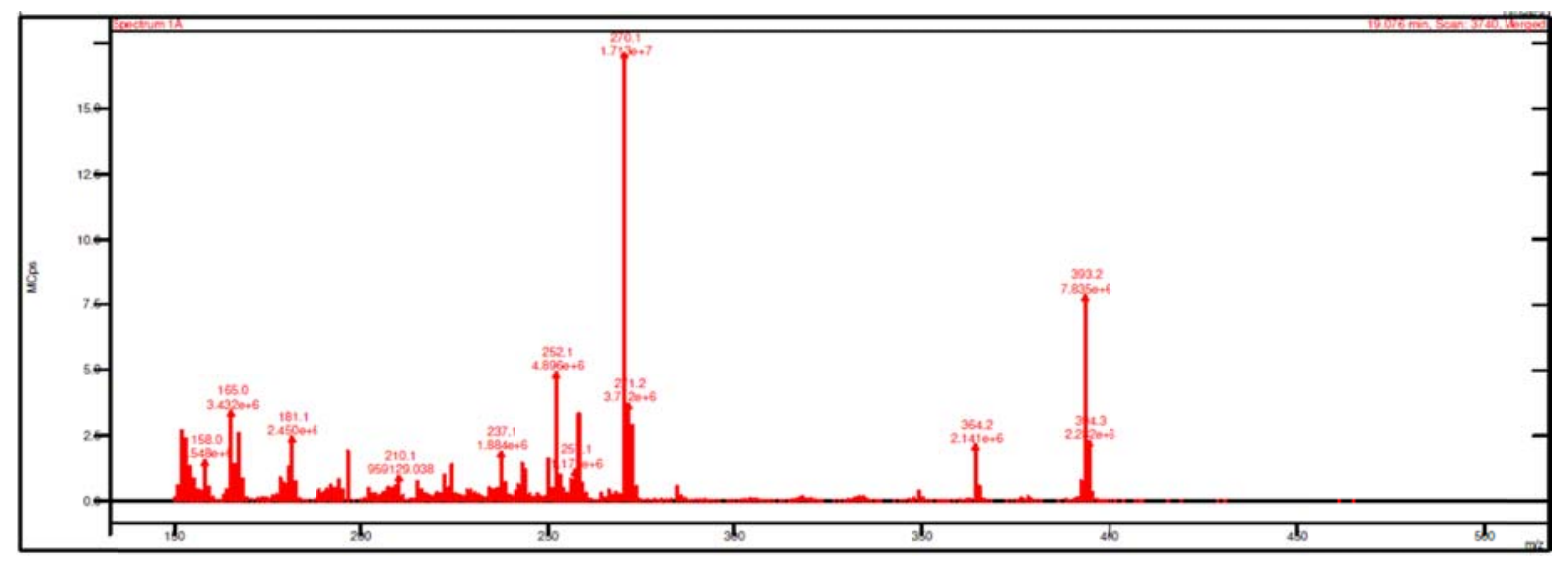

Figure S60. Mass spectra for cis-4-(4-methoxyphenyl)-3,6-dimethyl-2-(naphthalen-2-yl)1,2,3,4-tetrahydroquinoline (4t) 


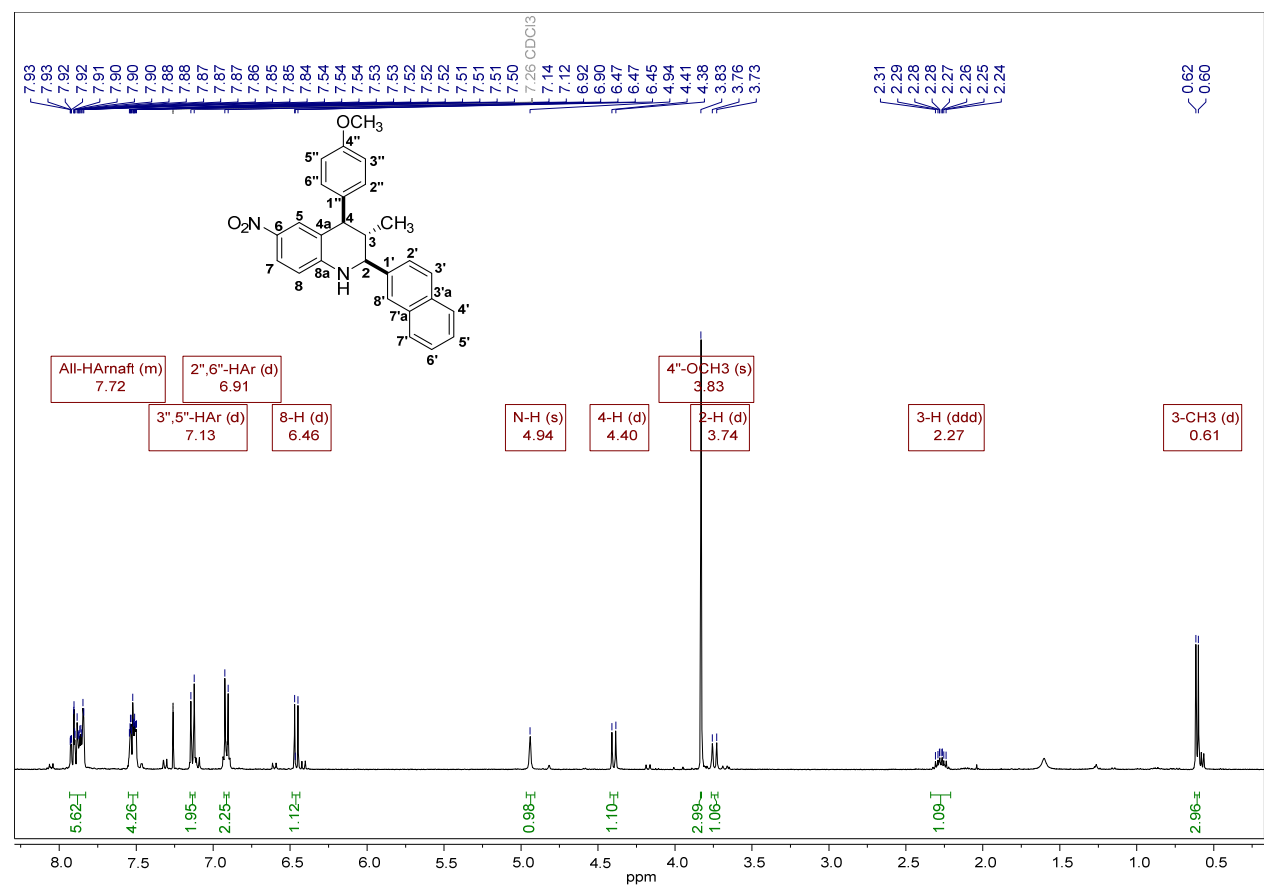

Figure S61. ${ }^{1} \mathrm{H}-\mathrm{NMR}$ spectra for cis-4-(4-methoxyphenyl)-3-methyl-2-(naphthalen-2-yl)-6nitro-1,2,3,4-tetrahydroquinoline (4u)

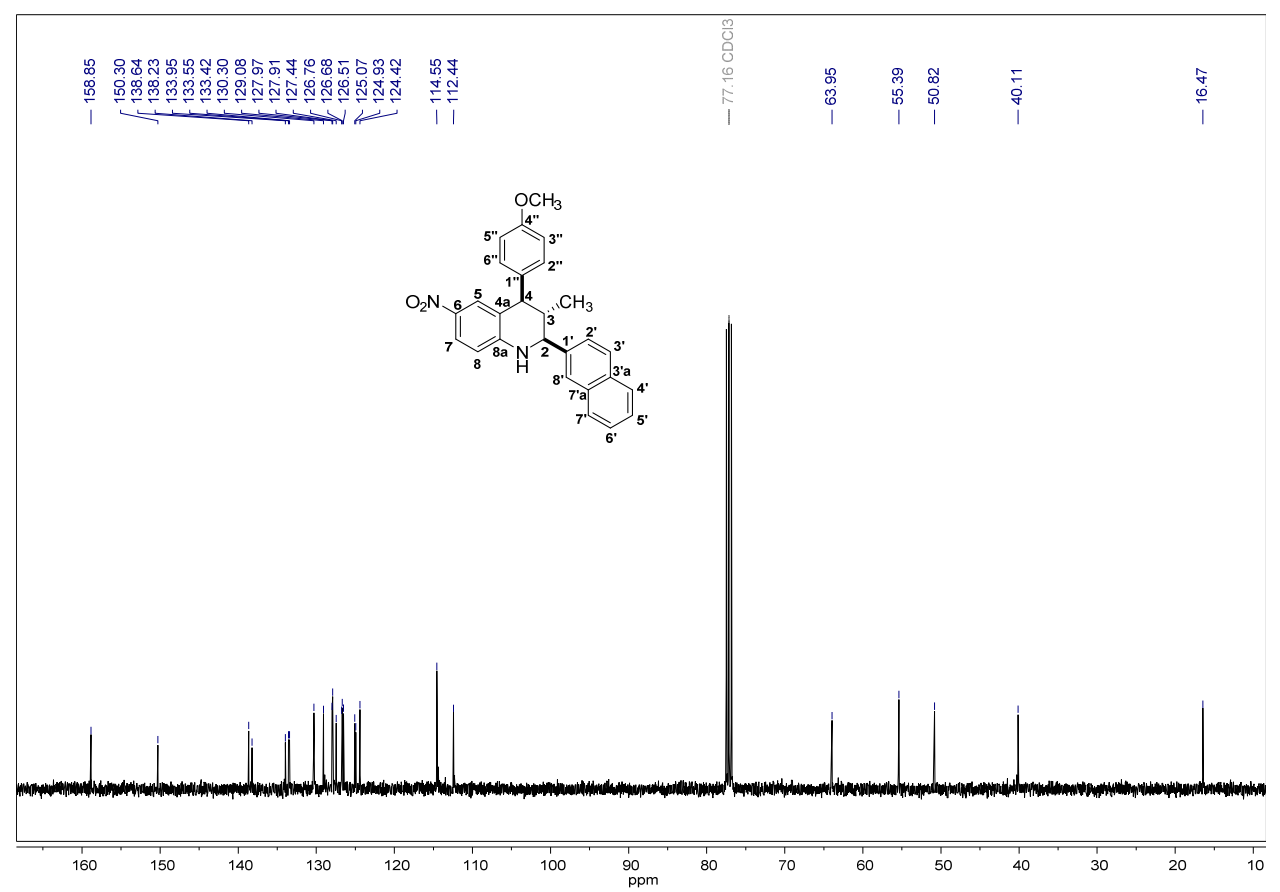

Figure S62. ${ }^{13} \mathrm{C}-\mathrm{NMR}$ spectra for cis-4-(4-methoxyphenyl)-3-methyl-2-(naphthalen-2-yl)6-nitro-1,2,3,4-tetrahydroquinoline (4u) 


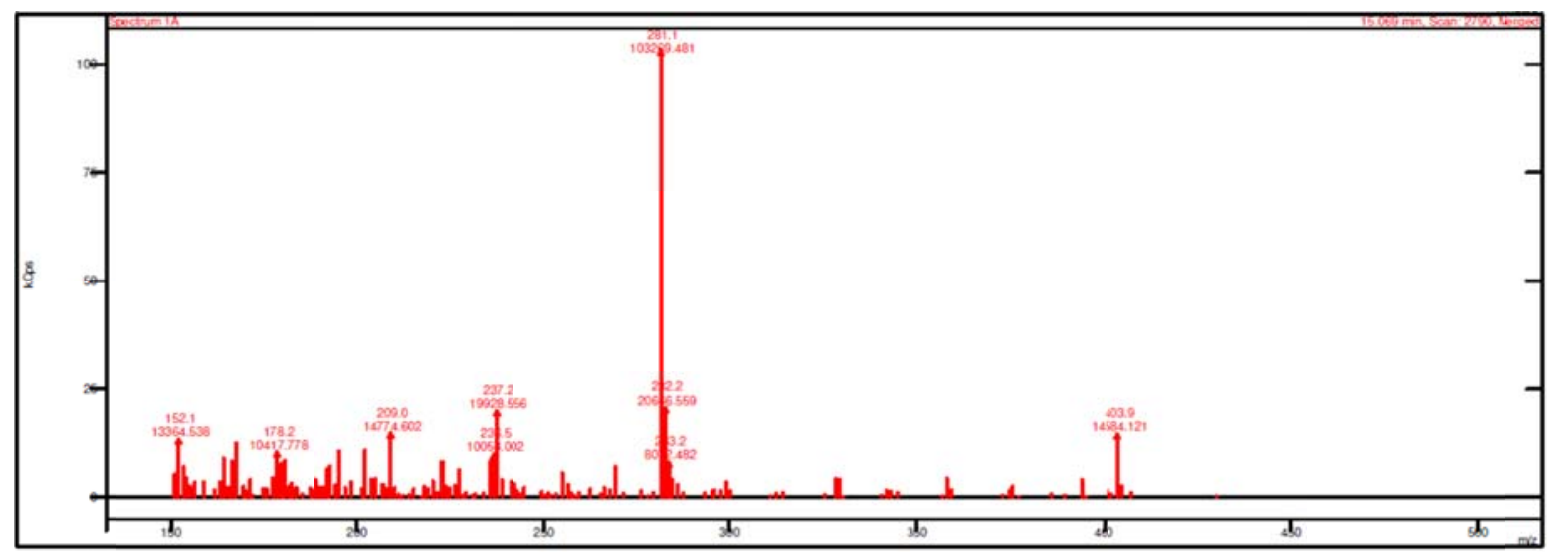

Figure S63. Mass spectra for cis-4-(4-methoxyphenyl)-3-methyl-2-(naphthalen-2-yl)-6nitro-1,2,3,4-tetrahydroquinoline (4u)

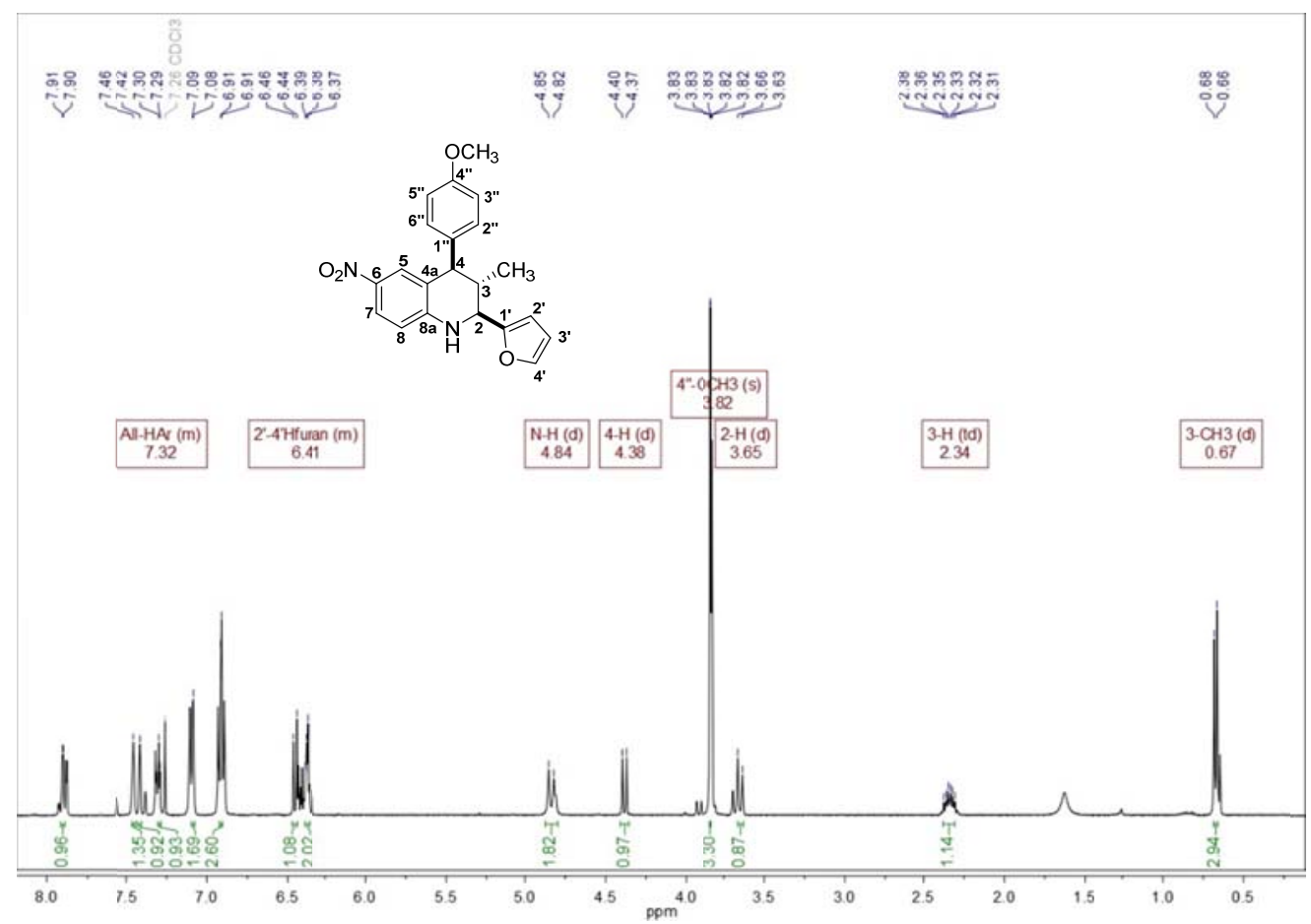

Figure S64. ${ }^{1}$ H-NMR spectra for cis-2-(furan-2-yl)-4-(4-methoxyphenyl)-3-methyl-6-nitro1,2,3,4-tetrahydroquinoline (4v) 


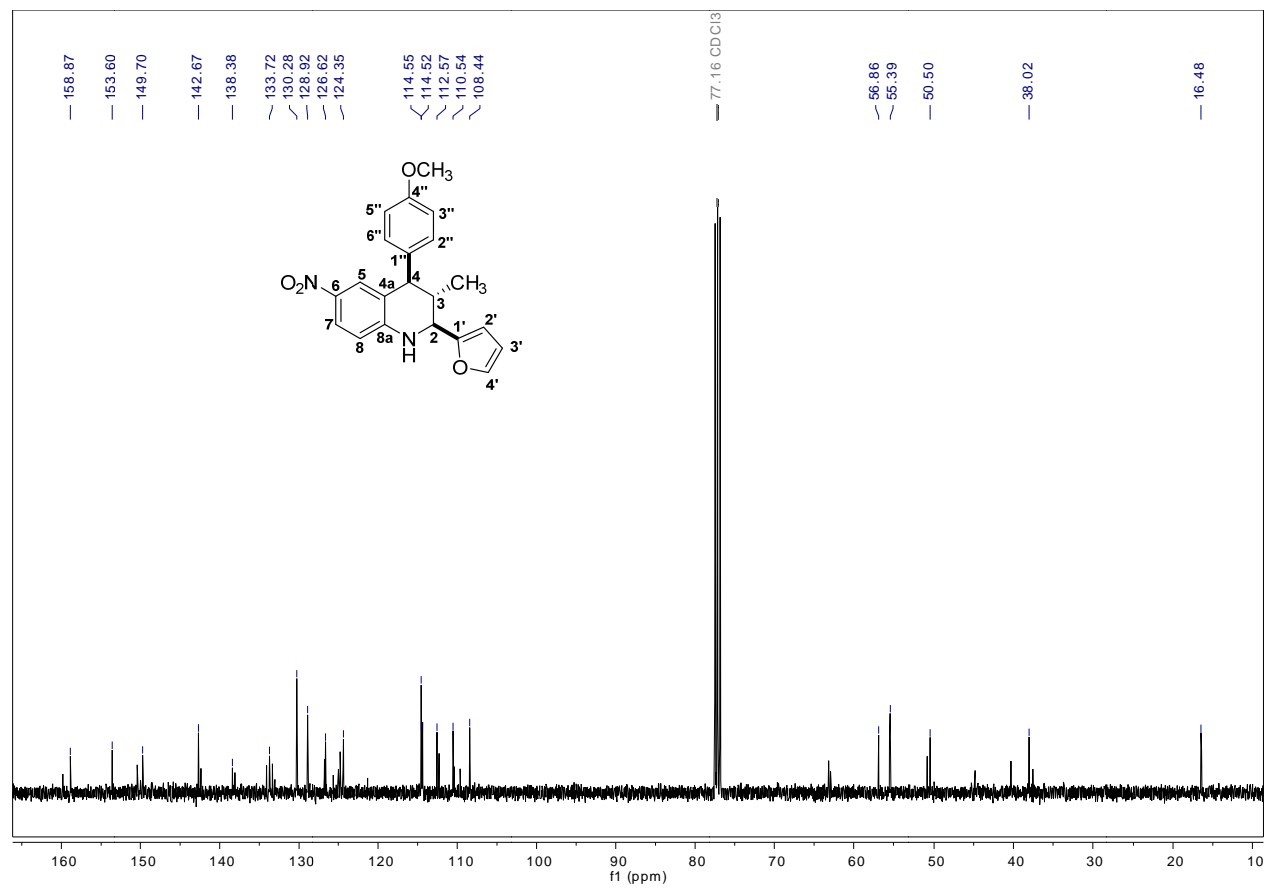

Figure S65. ${ }^{13}$ C-NMR spectra for cis-2-(furan-2-yl)-4-(4-methoxyphenyl)-3-methyl-6nitro-1,2,3,4-tetrahydroquinoline (4v)

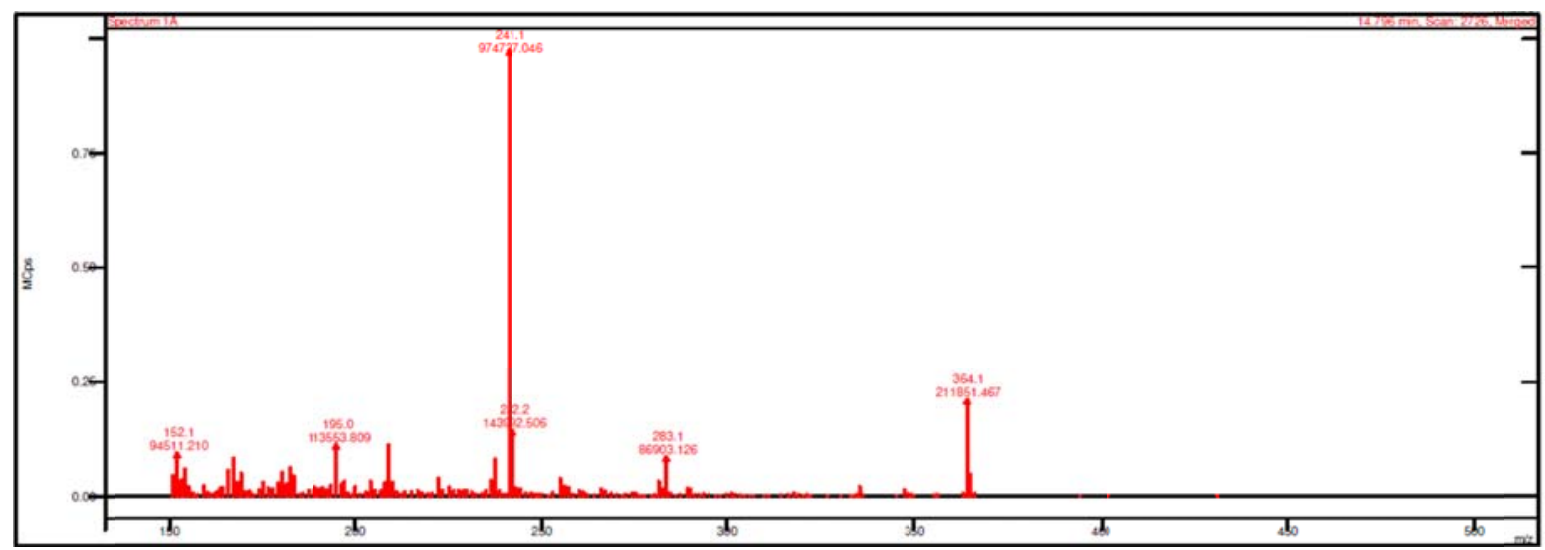

Figure S66. Mass spectra for cis-2-(furan-2-yl)-4-(4-methoxyphenyl)-3-methyl-6-nitro1,2,3,4-tetrahydroquinoline (4v) 


\section{Green chemistry metrics analysis}

The following formulae were used for calculating Atom Economy (AE), Atom Efficiency (AEf), Carbon Efficiency (CE), Reaction Mass Efficiency (RME), Optimum Efficiency (OE), Mass Productivity (MP), Mass Intensity (MI) and Process Mass Intensity (PMI), E factor, Solvent and Water Intensity (SI and WI).[1-4]

$$
\begin{aligned}
& \boldsymbol{A} \boldsymbol{E}=\frac{\text { Molecular weight of product }}{\text { Total molecular weight of reactants }} x 100 \\
& \boldsymbol{A} \boldsymbol{E} \boldsymbol{f}=\mathrm{AE} \times \text { yield } \% \\
& \boldsymbol{C E}=\frac{\text { Amount of carbon in the product }}{\text { total carbon present in reactants }} \times 100 \\
& \boldsymbol{R} \boldsymbol{M E}=\frac{\text { Mass of isolated product }}{\text { Total mass of reactants }} \chi 100 \\
& \boldsymbol{O} \boldsymbol{E}=\frac{\mathrm{RME}}{\mathrm{AE}} x 100 \\
& \boldsymbol{P} \boldsymbol{M I}=\frac{\text { Total mass of imput material in th whole process }}{\text { mass of product }} \\
& \boldsymbol{M P}=\frac{1}{\mathrm{PMI}} \chi 100 \\
& \text { E Factor }=\text { PMI }-1
\end{aligned}
$$



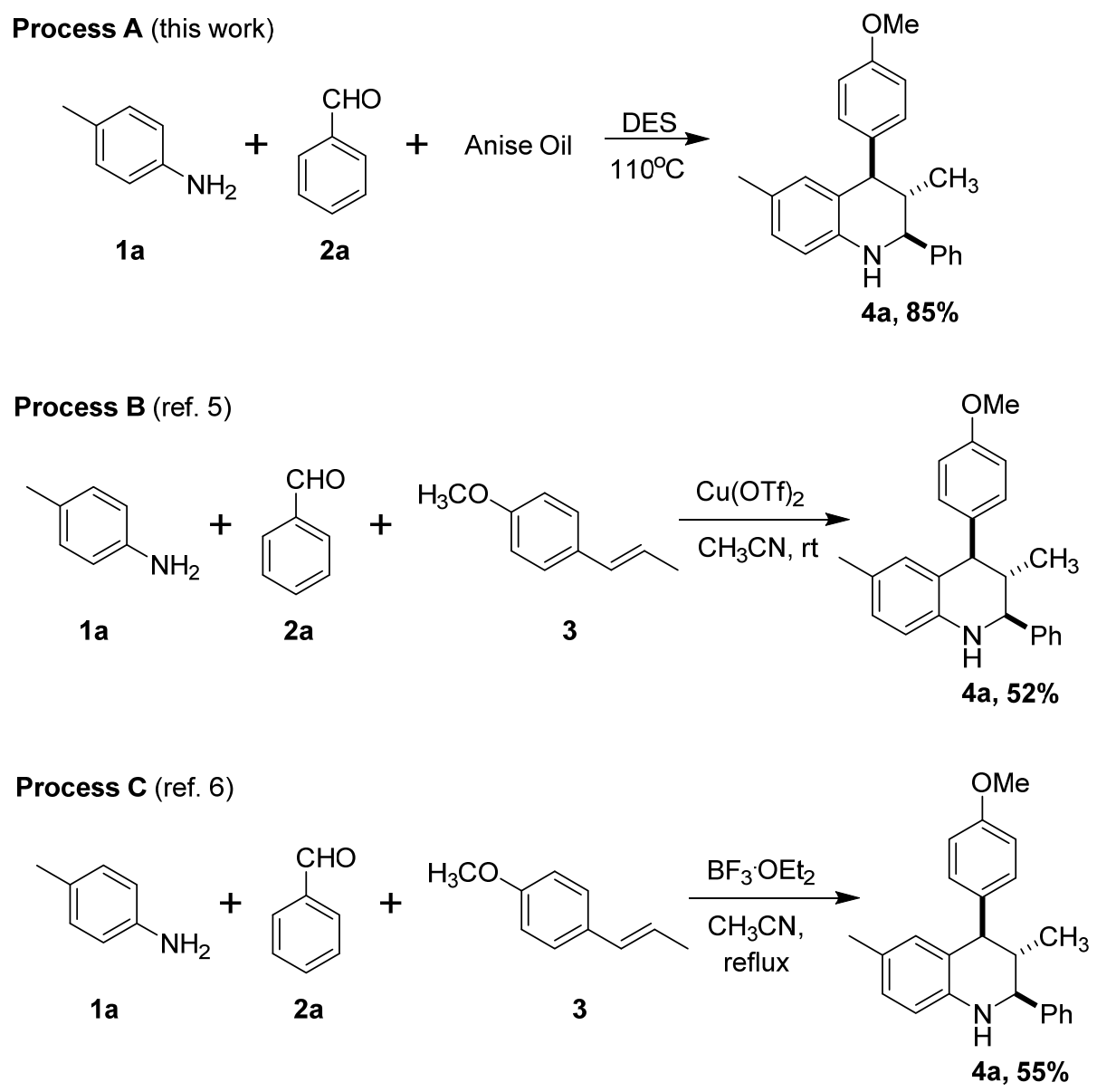

Scheme S1. Comparison of different synthetic methods for the obtention of compound 4a

\section{Current Method, Process A}

This is a one-step synthesis for the synthesis of compound $\mathbf{4 a}$ using commercial transanethole as shown in the model reaction. 
Process A (this work)<smiles>Cc1ccc(N)cc1</smiles>

1a

MW: 107,16

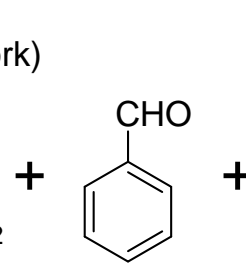

2a

MW: 106,12

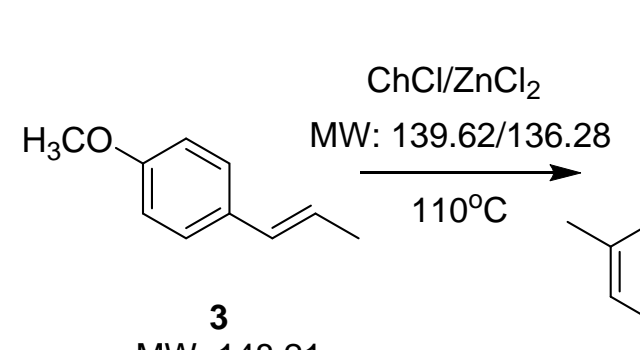

MW: 148,21<smiles>COc1ccc(C2c3ccccc3NC(c3ccccc3)[C@@H]2C)cc1</smiles>

$4 a, 85 \%$

MW: 343,47

Scheme S2. Synthesis of compound $4 a$ in $\mathrm{ChCl} / \mathrm{ZnCl}_{2} \mathrm{DES}$

General experimental procedure: $0.8 \mathrm{~g}$ of $\mathrm{ChCl} /$ zinc chloride DES (1.5:1) was heated to 70 ${ }^{\circ} \mathrm{C}$ to obtain a clear melt. To this melt a mixture of substituted aniline $(1 \mathrm{mmol})$, aromatic aldehyde $(1 \mathrm{mmol})$ and trans-anethole $(1 \mathrm{mmol}, 148.2 \mathrm{mg})$ was added and the reaction was stirred at $110{ }^{\circ} \mathrm{C}$ for $3 \mathrm{~h}$. After completion of the reaction (monitored by TLC), the reaction mixture was quenched by adding water while still hot, cooled to room temperature and the crude solid was filtered, washed with water (assuming use of total $5.0 \mathrm{~mL}$ ) and purified by column chromatography on silica gel (60-120 mesh) using a mixture of petroleum etherethyl acetate as eluent to afford the tetrahydroquinoline derivative 4a.

Materials used for metrics calculations: $p$-toluidine $(1 \mathrm{mmol}, 107.15 \mathrm{mg})$, benzaldehyde (1 mmol, $106.12 \mathrm{mg}), \mathrm{ChCl}$ (3.47 mmol, $484.83 \mathrm{mg}), \mathrm{ZnCl}_{2}(2.31 \mathrm{mmol}, 315.48 \mathrm{mg})$, transanethole (1 mmol, $148.21 \mathrm{mg})$, water $(5 \mathrm{~mL}, 5 \mathrm{~g})$, compound $4 \mathrm{a}(0.85 \mathrm{mmol}, 291.94 \mathrm{mg})$.

$$
\begin{aligned}
& \boldsymbol{A E}=\frac{343.47}{107.16+106.12+148.21} \times 100=95.01 \\
& \boldsymbol{A E} \boldsymbol{f}=\frac{95.01}{100} \times 85=80.75 \\
& \boldsymbol{C E}=\frac{24 \times 0.85}{(7 \times 1)+(7 \times 1)+(10 \times 1)} \times 100=85 \\
& \boldsymbol{R M} \boldsymbol{E}=\frac{291.94}{107.15+106.12+148.21} \times 100=80.76
\end{aligned}
$$




$$
\begin{aligned}
& \boldsymbol{O E}=\frac{80.76}{95.01} \times 100=85 \\
& \boldsymbol{P M I}=\frac{107.15+106.12+484.83+315.48+148.21+5000}{291.94}=21.10 \\
& \boldsymbol{M P}=\frac{100}{21.10}=4.73 \\
& \boldsymbol{E} \boldsymbol{F a c t o r}=4.73-1=3.73 \\
& \boldsymbol{S I}=\frac{484.83+315.48}{291.94}=2.74 \\
& \boldsymbol{W I}=\frac{5000}{291.94}=17.12
\end{aligned}
$$

\section{Published synthetic method - Process B}

This is a one-step synthesis, however the reported procedures for the synthesis of compound 4a do not contain all the required information; therefore, some realistic assumptions were used where appropriate. Drying agents, when used, were not included in the calculations.

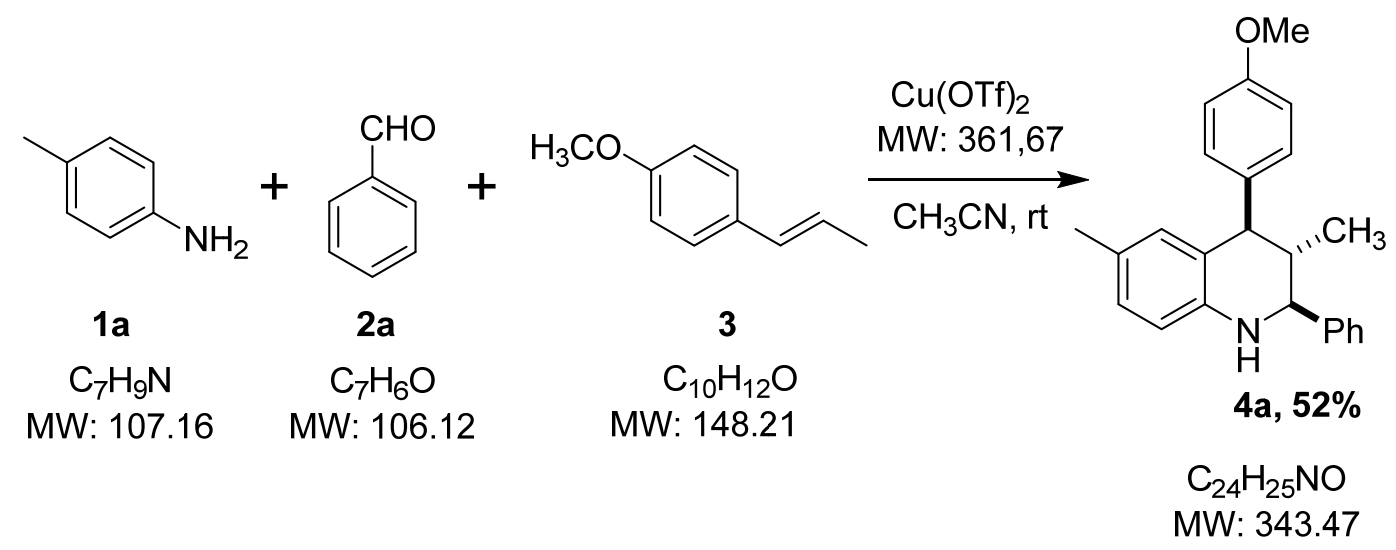

Scheme S3. Synthesis of compound 4a employing $\mathrm{Cu}(\mathrm{OTf})_{2}$ as catalyst.

Experimental procedure: A mixture of $p$-toluidine $(1 \mathrm{mmol})$ and benzaldehyde $(1.1 \mathrm{mmol})$ in $\mathrm{CH}_{3} \mathrm{CN}(10 \mathrm{~mL})$ was stirred at room temperature for $1 \mathrm{~h} .10 \mathrm{~mol} \% \mathrm{Cu}(\mathrm{OTf})_{2}$ as added in solution into the mixture. Finally, after $30 \mathrm{~min}$ the natural product (trans-anethole 1.3 
mmol) was added dropwise to the reaction mixture. The resulting mixture was stirred at room temperature for $16 \mathrm{~h}$ (overnight). After completion of the reaction as indicated by TLC, the reaction mixture was successively washed with water (assuming use of $20 \mathrm{~mL}$ ) and brine and extracted with ethyl acetate $(3 \times 15 \mathrm{~mL})$. The organic layer was separated and dried $\left(\mathrm{Na}_{2} \mathrm{SO}_{4}\right)$, concentrated in vacuo and the crude product was purified by column chromatography using silica gel (60-120 mesh) and eluted with hexane-ethyl acetate to afford pure tetrahydroquinoline $\mathbf{4 a}$.

Materials used for metrics calculations: $p$-toluidine $(1 \mathrm{mmol}, 107.15 \mathrm{mg})$, benzaldehyde (1.1 mmol, $116.73 \mathrm{mg}), \mathrm{CH}_{3} \mathrm{CN}(10 \mathrm{~mL}, 7.86 \mathrm{~g}), \mathrm{Cu}(\mathrm{OTf})_{2}(0.1 \mathrm{mmol}, 36.16 \mathrm{mg})$, transanethole (1.3 mmol, $192.67 \mathrm{mg})$, ethyl acetate $(45 \mathrm{~mL}, 40.59 \mathrm{~g})$, water $(20 \mathrm{~mL}, 20 \mathrm{~g})$, compound $4 \mathbf{a}(0.52 \mathrm{mmol}, 178.60 \mathrm{mg})$.

$$
\begin{aligned}
& \boldsymbol{A E}=\frac{343.47}{107.16+106.12+148.21} \times 100=95.01 \\
& \boldsymbol{A E} \boldsymbol{f}=\frac{95.01}{100} \times 52=49.40 \\
& \boldsymbol{C E}=\frac{24 \times 0.52}{(7 \times 1)+(7 \times 1.1)+(10 \times 1.3)} \times 100=45.05 \\
& \boldsymbol{R M} \boldsymbol{E}=\frac{178.60}{107.15+116.73+192.67} \times 100=42.87 \\
& \boldsymbol{O E}=\frac{42.87}{95.01} \times 100=45.12 \\
& \boldsymbol{P M I}=\frac{107.15+116.73+7860+36.16+192.67+40590+20000}{178.60}=385.79 \\
& \boldsymbol{M P}=\frac{100}{385.79}=0.25 \\
& \boldsymbol{E} \boldsymbol{F a c t o r}=25.92-1=24.92 \\
& \boldsymbol{S I}=\frac{7860+40590}{178.6}=271.27 \\
& \boldsymbol{W I}=\frac{20000}{178.6}=111.98
\end{aligned}
$$




\section{Published synthetic method - Process C}

This is a one-step synthesis, however the reported procedures for the synthesis of compound 4a do not contain all the required information; therefore, some realistic assumptions were used where appropriate. Drying agents, when used, were not included in the calculations.

Process C (ref. 55)

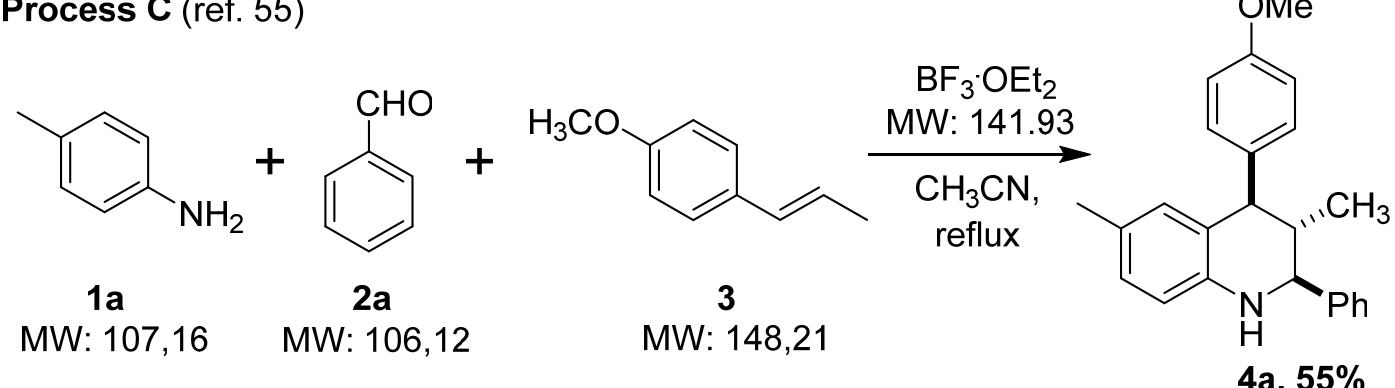

MW: 343,47

Scheme S4. Synthesis of compound 4a employing $\mathrm{BF}_{3} \cdot \mathrm{OEt}_{2}$ as catalyst.

Experimental procedure for compound $4 \boldsymbol{a}$ : $\mathrm{BF}_{3} \mathrm{OEt}_{2}(0.61 \mathrm{~g}, 4.30 \mathrm{mmol})$ was added to a mixture of $p$-toluidine $(0.460 \mathrm{~g}, 4.30 \mathrm{mmol})$ and benzaldehyde $(0.50 \mathrm{~g}, 4.73 \mathrm{mmol})$ in anhydrous $\mathrm{CH}_{3} \mathrm{CN}(15 \mathrm{~mL})$, stirred at room temperature for $30 \mathrm{~min}$. Over a period of 20 min, a solution of commercial trans-anethole $(0.84 \mathrm{~g}, 5.68 \mathrm{mmol})$ in $\mathrm{CH}_{3} \mathrm{CN}(10 \mathrm{~mL})$ was added dropwise. The resulting mixture was stirred at $70{ }^{\circ} \mathrm{C}$ for $10 \mathrm{~h}$. After completion of the reaction, as indicated by TLC, the reaction mixture was diluted with water $(30 \mathrm{~mL})$ and extracted with ethyl acetate $(3 \times 15 \mathrm{~mL})$. The organic layer was separated and dried $\left(\mathrm{Na}_{2} \mathrm{SO}_{4}\right)$, concentrated in vacuo and the crude product was purified by column chromatography using silica gel (60-120 mesh) and eluted with petroleum ether-ethyl acetate to afford pure tetrahydroquinoline $\mathbf{4 a}$. 
Materials used for metrics calculations: p-toluidine (4.3 mmol, $460.74 \mathrm{mg})$, benzaldehyde (4.73 mmol, $501.94 \mathrm{mg}), \mathrm{CH}_{3} \mathrm{CN}(25 \mathrm{~mL}, 19.65 \mathrm{~g}), \mathrm{BF}_{3} \cdot \mathrm{OEt}_{2}$ (4.30mmol, $\left.610 \mathrm{mg}\right)$, transanethole $(5.68 \mathrm{mmol}, 841.8 \mathrm{mg})$, ethyl acetate $(45 \mathrm{~mL}, 40.59 \mathrm{~g})$, water $(30 \mathrm{~mL}, 30 \mathrm{~g})$, compound 4a (2.36 mmol, $812.30 \mathrm{mg})$.

$$
\begin{aligned}
& \boldsymbol{A E}=\frac{343.47}{107.16+106.12+148.21} \times 100=95.01 \\
& \boldsymbol{A E} \boldsymbol{f}=\frac{95.01}{100} \times 55=52.25 \\
& \boldsymbol{C} \boldsymbol{E}=\frac{24 \times 2.36}{(7 \times 4.3)+(7 \times 4.73)+(10 \times 5.68)} \times 100=47.19 \\
& \boldsymbol{R} \boldsymbol{M E}=\frac{812.30}{460.74+501.94+841.8} \times 100=45.01 \\
& \boldsymbol{O E}=\frac{45.01}{95.01} \times 100=47.37 \\
& \boldsymbol{P M I}=\frac{460.74+501.94+19650+610+841.8+40590+30000}{812.30}=114.06 \\
& \boldsymbol{M P}=\frac{100}{114.06}=0.87 \\
& \boldsymbol{E} \boldsymbol{F a c t o r}=114.06-1=113.06 \\
& \boldsymbol{S I}=\frac{19650+40590}{812.30}=74.15 \\
& \boldsymbol{W I}=\frac{30000}{812.30}=36.93
\end{aligned}
$$




\section{References}

1. Constable, D. J. C.; Curzons, A. D.; Cunningham, V. L., Metrics to 'green' chemistry—which are the best? Green Chem. 2002, 4, (6), 521-527.

2. Augé, J., A new rationale of reaction metrics for green chemistry. Mathematical expression of the environmental impact factor of chemical processes. Green Chem. 2008, $10,(2), 225-231$.

3. Jiménez-González, C.; Constable, D. J. C.; Ponder, C. S., Evaluating the "Greenness" of chemical processes and products in the pharmaceutical industry-a green metrics primer. Chem. Soc. Rev. 2012, 41, (4), 1485-1498.

4. Jimenez-Gonzalez, C.; Ponder, C. S.; Broxterman, Q. B.; Manley, J. B., Using the Right Green Yardstick: Why Process Mass Intensity Is Used in the Pharmaceutical Industry To Drive More Sustainable Processes. Org. Process Res. Dev. 2011, 15, (4), 912-917.

5. Romero Bohorquez, A. R.; Kouznetsov, V. V.; Doyle, M. P., Cu(OTf) 2 -Catalyzed Three-Component Imino Diels-Alder Reaction Using Propenylbenzenes: Synthesis of 2,4Diaryl Tetrahydroquinoline Derivatives. Lett. Org. Chem. 2011, 8, (1), 5-11.

6. Romero Bohorquez, A. R.; Rivero, P. E.; Leal, S., M.; Kouznetsov, V. V., In Vitro Activity Against Trypanosoma cruzi and Leishmania chagasi Parasites of 2,4-Diaryl 1,2,3,4-Tetrahydroquinoline Derivatives. Lett. Drug Des. Discov. 2012, 9, (8), 802-808. 